LBL-8031 C. 2

UC -37

\title{
ZEEMAN ATOMIC ABSORPTION SPECTROMETRY
}

RECEIVED

Ted Hadeishi and Ralph McLaughlin

August 1978
LAWRENCE
BERKELEY LABORATORY

SEP 251978

LIBRARY AND

DOCUMENTS SECTION

Prepared for the National Science Foundation - RANN and the $U$. S. Department of Energy under Contract W-7405-ENG-48

\section{TWO-WEEK LOAN COPY}

\section{This is a Library Circulating Copy}

which may be borrowed for two weeks.

For a personal retention copy, call

Tech. Info. Division, Ext. 6782

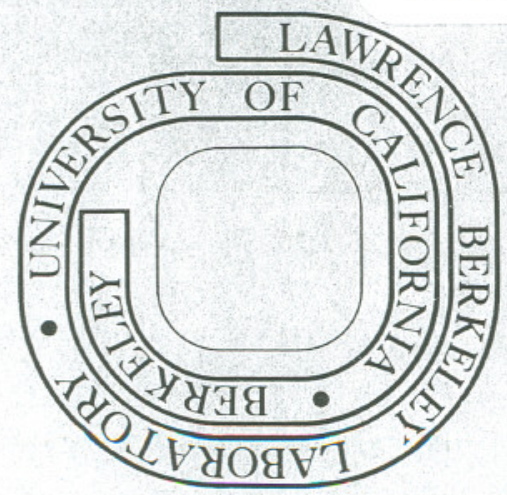




\section{LEGAL NOTICE}

This report was prepared as an account of work sponsored by the United States Government. Neither the United States nor the Department of Energy, nor any of their employees, nor any of their contractors, subcontractors, or their employees, makes any warranty, express or implied, or assumes any legal liability or responsibility for the accuracy, completeness or usefulness of any information, apparatus, product or process disclosed ar renraconts that its use would not:

Lawrence Berkeley Laboratory Library

University of California, Berkeley 
Final Report

ZEEMAN ATOMIC ABSORPTION SPECTROMETRY

Ted Hadei shi

and

Ralph McLaughl in 
I. Introduction ....................

II. Use of Zeeman Effect as a Solution to Problems of Atomic Absorption Spectrometry . . . . . . . . . . . .

III. Operating Principle of ZAA . . . . . . . . . . .

IV. Technique of Direct Sample Atomization for Determination of Trace Elements...................

V. Magnetically Confined Lamp (MCL) . . . . . . . . .
A. Description ..................
B. Construction ..................
C. Preparation of Cathodes .............

VI. Sensitivity

VII. Sample Manipulation
A. Introduction
B. Matrix Modification
C. Furnace Effects
D. Experience with Specific Elements

VIII. Conclusions . References

Appendix: ZAA Instruction Manual ............ 


\section{Introduction}

In the year 1970, a group of scientist concerned about environmental problems started a weekly seminar to search for possible areas of application of basic science. This was also the year in which the Minomata Bay mercury poisoning incident was attracting world wide attention. The present principal investigator (PI) was one member of the audience at the weekly seminars and became interested in the possibility of doing something about the mercury problem. He started to obtain information concerning the methods available to make quantitative determination of the presence of mercury. The PI, having obtained his training in physics, was quite surprised to find out that atomic absorption spectrometry was used for trace elemental detection. At the same time, because of basic research experience in atomic physics dealing with atomic absorption and fluorescence phenomena, it was immediately obvious that conventional atomic absorption spectrometry was inadequate to measure the mercury content directly from the host material because of background interference. It was also quite evident that all other elemental analyses by atomic absorption spectrometry were inadequate if it was desired to make the measurement directly on the host material.

It is generally true that in order to extract information buried in noise, whether it is a radio communication or an atomic physics signal one must design a detector tht achieves high specificity by means of extremely narrow banding as is done by ultra high resolution spectroscopy or radio frequency resonance spectroscopy. In the case 
of atomic absorption spectrometry for trace elements in the presence of large background noise, the necessary requirements are (1) high specificity and (2) high sensitivity. In general, conditions satisfying requirement (1) result in reduced sensitivity because of requirement (2) and vice versa.

The first task was to construct an atomic absorption spectrophotometer that satisfied both conditions (1) and (2) simultaneously. This was accomplished by means of a differential absorption measurement using a Zeeman shifted light source. The measurement depended upon obtaining the difference or ratio of absorption between unshifted zeeman components and shifted Zeeman components. High specificity, condition (1), was satisfied because the polarization dependence of Zeeman components can be used to achieve narrow banding. This is possible since the unshifted comonents can be selected when the light polarized parallel to the magnetic field is passed by a properly oriented polarizer while the shifted components can be obtained by passing the light through a polarizer oriented at $90^{\circ}$ with respect to the position that passed unshifted components. The width of the shifted and unshifted spectral lines are only determined by the characteristics of the lamp and NOT by a wave length selective element such as a monochromator. Thus, with an organic dye polarizer, as much as a $30^{\circ}$ solid acceptance angle can be used, and even with a Rochon polarizer, as much as $6^{\circ}$ of solid acceptance angle can be used. This results in high flux transmission through the detector system allowing one to achieve high sensitivity because of higher signal to noise $(\mathrm{S} / \mathrm{N})$ ratio. 
Using these concepts, the first Zeeman atomic absorption spectrometer was conceived and invented at LBL for the determination of mercury. ${ }^{1-5}$ This detector used a special furnace that was designed to atomize mercury in the host material whether it was solid, liquid or gas or whether the mercury was in the form of a metal oxide or metal organic compound. This instrument satisfied all of our initial expectations.

After completion of the mercury detector, the same basic principle was applied to the construction of a detector for other elements with a background correction capability similar to that of mercury. Our aim was to demonstrate to commercial manufacturers the "proof of concept" of Zeeman Atomic Absorption (ZAA) Spectrometry so that various ZAA models suitable for users having a variety of applications would become commercially available. The ZAA instrument in the present form does not satisfy the ultimate goal for a detector--a black box in which a completely inexperienced person can place a sample and obtain a read out of the amount of all the elements in that substance. However, we believe we have made a significant contribution to the first step in the right direction. 
II. Use of Zeeman Effect as a Solution to the Problem of Atomic Absorption Spectrometry

In order to accurately measure the concentration of an element directly from a sample by atomic absorption spectrometry the following two conditions must be satisfied:

1. Complete rejection of background interference and

2. Complete dissociation of the sample to atomic vapor of the element to be measured.

In order to completely dissociate a material into atomic vapor, one should not use either drying or ashing. The temperature of the sample should be rapidly raised to atomization temperature before the vapor enters into the absorption region. Since there are many metal organic compounds which have appreciable vapor pressures even below $100^{\circ} \mathrm{C}$, strictly speaking, the sample should not ever be dried.

However, associated with the rapid increase of the sample to a high temperature is the large amount of smoke and vapor which attenuates the light beam. Therefore, extremely accurate background correction capability is essential if one is to measure elements directly from completely unknown samples. No conventional atomic absorption spectrometer can correct for such background interference while zAA can handle attenuation of incident light to at least 2 absorbance units, i.e., 958 attenuation. ${ }^{6}$ This value should not be confused with similar quoted values for conventional AA units. Background correction at 2 absorbance units refers to non-uniform attenuation of light by a time varying interference, not just a uniform attenuation of the light beam. It is easy to show that in a conventional background corrected $\mathrm{AA}$, such 
as those that utilize a $\mathrm{D}_{2}$ lamp corrector, incomplete correction against non-uniform, time varying interfernce results since it is not possible to construct a resonance lamp (usually hollow cathode lamp or EDL) to be identical to the $\mathrm{D}_{2}$ correction lamp in terms of the intensity and (an even more severe condition) in terms of the intensity distribution. Unless these two lamps are identical, in spacial and temporal intensity distribution, an error is introduced when correcting for a spacial and temporal varying background.

Thus, it is obvious that the background correction light and the resonance radiation light must be as identical as possible. In order to be identical, it must be only one light source. Mother Nature provides us with just such a light source. This is furnished by the Zeeman Effect. The Zeeman effect occurs either when a magnetic field is placed about the light source or about the abosrbing atomic vapor. Both effects are identical. Let us first consider a case in which a strong magnetic field is applied to the light source. Resonance radiation emitted in a direction normal to the magnetic field is split due to the Zeeman effect into several components with different wave-lengths. Those components polarized parallel to the magnetic field ( $\pi$ components) are normally not shifted or are only shifted by a small amount compared to those components polarized perpendicular to the magnetic field ( $\sigma$ components). Note, also, that for the simple spectral transition that occurs in $\mathrm{Ag}, \mathrm{Se}, \mathrm{Cd}, \mathrm{Zn}, \mathrm{Pb}, \mathrm{Hg}$ etc., the $\pi$ component is not shifted at all and that the $\sigma^{+}$and $\sigma^{-}$components are shifted symmetrically about the single $\pi$ component. However, since the $\pi$ and $\sigma$ components are all emitted from the same light source, the temporal and spacial 
distribution of the intensity is identical, thus satisfying the requirement of the ideal background corrector.

In the actual instrument, the $\pi$ and $\sigma$ components pass through the atomic vapor alternately and the difference in absorption or ratio of absorption between the components is measured hence rejecting the background interference completely. This is true since the background interference influences both $\pi$ and $\sigma$ components equally.

However the ZAA background corrector above is completely useless unless one can atomize the sample in the same way as the calibration standard. It is, however, quite obvious how this can be accomplished. If a sample, (solid, liquid or gas) is suddenly introduced into an atomization furnace already held at atomization temperature and the design is such that no nonatomized evaporated vapor gets into the absorption section of furnace, then regardless of the sample matrix, one should be able to measure the amount of trace element present. of course, gas flow dynamics must be taken into account since when the solid or liquid suddently goes into the gas phase, a change in flow rate occurs.

A ZAA instrument with such a furnace ${ }^{7}$ was demonstrated to perform analyses that were indpendent of the sample matrix. A number of solid and liquid samples were determined directly in this instrument without chemical pretreatment. 


\section{Operating Principles of ZAA}

The Zeeman effect was discovered in $1897,{ }^{8}$ theoretically interpreted by Heisenberg, ${ }^{9}$ in 1926 and is one of the most well understood phenomenon in atomic physics. The zeeman effect is a most useful tool for term analysis in atomic spectroscopy because the theory so accurately represents the experimental observations.

We have decided to utilize this most well understood phenomenon for background correction. Monitoring and reference wave-lengths are obtained from the light source in a magnetic field by using the $\pi$ and $\gamma$ components. The following simple discussion ${ }^{10}$ shows the operating principle of $\mathrm{ZAA}$ technique.

In the presence of a magnetic field of strength $\mathrm{H}$ in the direction of the $\mathrm{z}$ - axis, the Hamiltonian representing the interaction between the electrons in an atom and the field is given by

$$
\mathfrak{H}^{M}=\frac{e H}{2 \mu C} \sum_{i=1}^{N}\left(L_{z i}+2 S_{z i}\right)=\frac{e H}{2 \mu C}\left(L_{z}+2 S_{z}\right)
$$

The whole Zeeman phenomenon (the effect of magnetic fields on atomic and molecular emission) can be understood by a study of this perturbation Hamil toni an .

Before describing ZAA from a quantum mechanical point of view, let us consider the zeeman effect from a classical point of view. A description of the anomalous Zeeman effect requires the introduction of the spin quantum number $S$; as was done by Heisenberg. For the time being let us ignore the electron spin and describe atoms only in terms of a purely orbital scheme so that the entire angular momentum 
of the atom is given by the sum of the $\overline{\mathrm{L}}$ vectors for the individual electrons. In such a scheme $\mathrm{L}_{z}$, the sum of the $\mathrm{z}$-components of the orbital angular momenta is a constant of motion and is given by $M \hbar$ where $M$ has quantized integral value such that $-\mathrm{L} \leq \mathrm{M} \leq \mathrm{L}$. The emission of spectral lines from such an atom is due to the "quantum jump" of the electric dipole transition. For $\Delta M_{L}=0$, the light is linearly polarized with the electric vector in the plane determined by the $\mathrm{z}$-axis and the direction of propagation. For the $\Delta M_{L}= \pm 1$ case, the radiation is circularly polarized when viewed along the z-axis and hence linearly polarized perpendicular to the Z-axis when viewed in the plane determined by remaining two orthonormal axes. The transition in which $\Delta \mathrm{M}_{L}=0$ will have the same frequency as the unperturbed line. The transition for whch $M_{L} \rightarrow M_{L}+1$ on emission will be shifted toward lower frequency from the unperturbed line by an amount

$$
\left\{\frac{\mathrm{eH}}{2 \mu \mathrm{c}} / 2 \pi\right\} \sec ^{-1} \text { or }(\mathrm{eH} / 2 \pi \mathrm{c}) \mathrm{cm}^{-1}
$$

The transition for which $\mathrm{M}_{L} \rightarrow \mathrm{M}_{L}-1$ will be shifted toward higher frequency by the same amount. Figure 1 (Ref. 11) shows the polarization effect of emitted light. The case for abosrbed light is the complete opposite of emitted light with regard to the shift in frequency and direction of circular polarization.

Now let us consider the Zeeman effect from the quantum mechanical point of view including electron spin. For $\mathrm{ZAA}$, the magnetic field strength used is less than $24 \mathrm{KG}$, so that the weak-field case can be used. One needs to consider the intermediate field use (Paschen-Bach case) only 


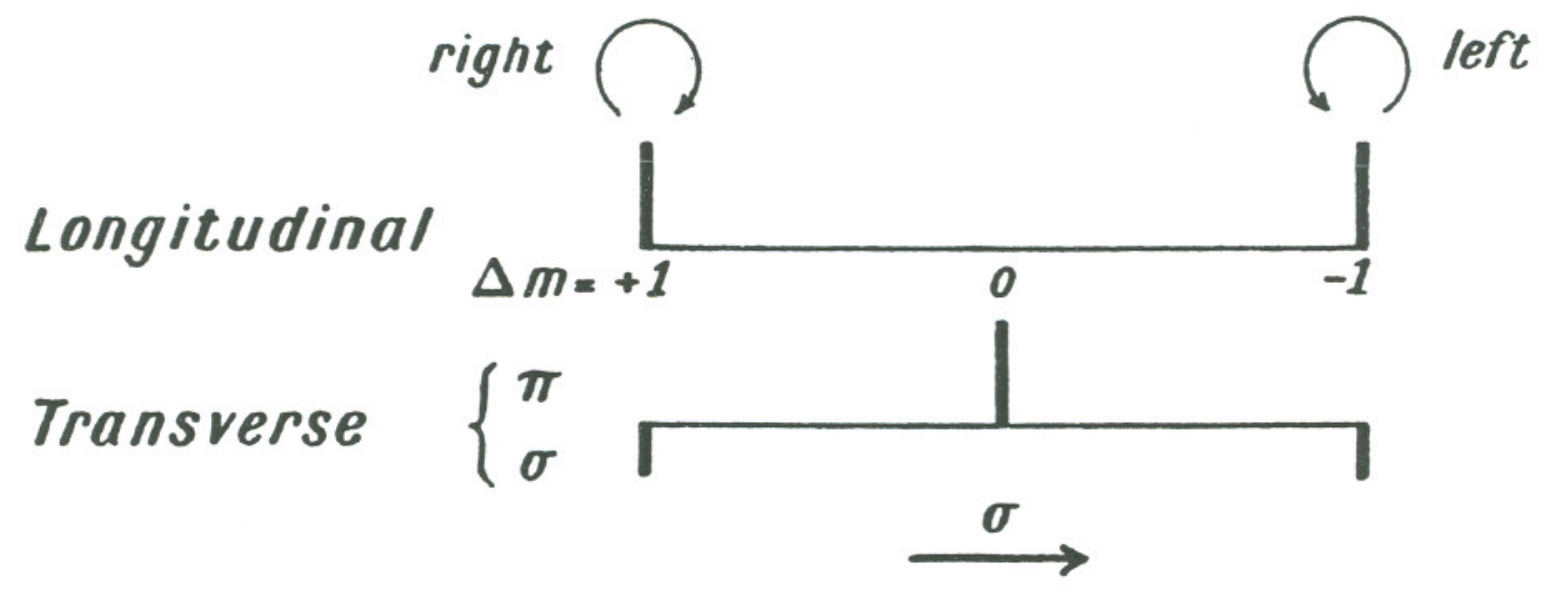

XBL 788-9873

Fig. 1. Polarization of Zeeman components. If look parallel to field components are circularly polarized. If look Transverse $\pi$ components show linear polrization parallel to field and $\sigma$ components show linear polarization perpendicular to field. 
when $\mathrm{eH} / 2 \mu \mathrm{c} \sim \zeta(\overline{\mathrm{L}} \cdot \overline{\mathrm{S}})$ (where $\zeta$ is the fine structure splitting constant). This requires much higher field strengths for most atoms. In the Russel-Saunders case, the atomic wave-function can be characterized by SLJ and that part of the matrix of $\mathcal{H}^{\mathrm{M}}$ which corresponds to this energy level.

The diagonal matrix element of the perturbation energy has value

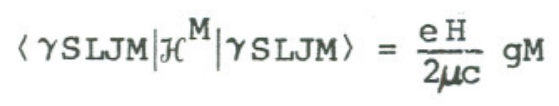

where

$$
g(\mathrm{SLJ})=1+\frac{\mathrm{J}(\mathrm{J}+1)-(\mathrm{L}+1)+\mathrm{S}(\mathrm{S}+1)}{2 \mathrm{~J}(\mathrm{~J}+1)}
$$

For the intermediate coupling case in which Russel-Saunders coupling no longer holds, the matrix element, in general, has the form

$$
\left\langle\gamma j_{l} J_{p^{J}} M_{j}\left|H_{C}^{M}\right| \gamma_{j l} J_{p^{J} M_{J}}\right\rangle=\frac{e^{H}}{2 \mu C} g M
$$

Here

$$
\begin{aligned}
g=g & \frac{J(J+1)+j_{1}\left(j_{1}+1\right)-J_{p}\left(J_{p}+1\right)}{2 J(J+1)} \\
& +g_{p} \frac{J(J+1)+J_{p}\left(J_{p}+1\right)-j_{1}\left(j_{1}+1\right)}{2 J(J+1)}
\end{aligned}
$$

It is important to note that regardless of the coupling scheme, 
the Lande $g$ value is a constant. Because of this the splitting is more or less alike. Now, we shall compute intensities of the Zeeman pattern. THE PATTERN IS INDEPENDENT OF COUPLING SCHEME!!!

For the Zeeman pattern of any line in which there is no change in $\mathrm{J}$, the strengths of the components in the transverse direction $(\theta=\pi / 2)$ are proportional to

$$
\begin{aligned}
& \begin{aligned}
&\left|\left\langle\alpha \mathrm{JM}|\overline{\mathrm{P}}| \alpha^{1} \mathrm{JM}\right\rangle\right|^{2}=\left|\left\langle\alpha \mathrm{J}\|\mathrm{P}\| \alpha^{1} \mathrm{~J}\right\rangle\right|^{2} \mathrm{M}^{2} \text { for } \pi \text { components } \\
& 1 / 2\left|\left\langle\alpha \mathrm{JM}|\overline{\mathrm{P}}| \alpha^{1} \mathrm{JM} \mp \mathrm{I}\right\rangle\right|^{2} \\
& \quad=\left|\left\langle\alpha \mathrm{J}\|\mathrm{P}\| \alpha^{1} \mathrm{~J}\right\rangle\right|^{2} 1 / 4(\mathrm{~J} \pm \mathrm{M})(\mathrm{J} \mp \mathrm{M}+1) \quad \text { for } \sigma \text { component }
\end{aligned}
\end{aligned}
$$

Similarly for $\mathrm{J} \rightarrow \mathrm{J}+1$ and $\mathrm{J} \rightarrow \mathrm{J}-1$ we have

$$
\left|\left\langle\alpha \mathrm{JM}|\overline{\mathrm{P}}| \alpha^{1} \mathrm{~J}+1 \mathrm{M}\right\rangle\right|^{2}
$$

$$
=\left|\left\langle\alpha J\|P\| \alpha^{1} J+1\right\rangle\right|^{2}\left[(J+1)^{2}-M^{2}\right]
$$

$1 / 2\left|\left\langle\alpha \mathrm{JM}|\overline{\mathrm{P}}| \alpha^{1} \mathrm{~J}+1 \mathrm{M} \mp 1\right\rangle\right|^{2}$

$$
=\left|\left\langle\alpha J\|P\| \alpha^{1} J-1\right\rangle\right|^{2} 1 / 4(J+M \mp 1)(J+M \mp 2)
$$

and

$$
\left|\left\langle\alpha J M|\overline{\mathrm{P}}| \alpha^{1} \mathrm{~J}-\mathrm{IM}\right\rangle\right|^{2}=\left|\left\langle\alpha \mathrm{J}\|\mathrm{P}\| \alpha^{1} \mathrm{~J}-1\right\rangle\right|^{2}\left(\mathrm{~J}^{2}-\mathrm{M}^{2}\right)
$$


$1 / 2\left|\left\langle\alpha \mathrm{JM}|\overline{\mathrm{P}}| \alpha^{1} \mathrm{~J}-1 \mathrm{M} \overline{+} 1\right\rangle\right|^{2}=\left|\left\langle\alpha \mathrm{J}\|\mathrm{P}\| \alpha^{1} \mathrm{~J}-1\right\rangle\right|^{2} 1 / 4(\mathrm{~J} \pm \mathrm{M})(\mathrm{J} \pm \mathrm{M}-1)$

Here $\langle A\|P\| B\rangle$ is a reduced matrix element.

An example of the intensity distribution of the Zeeman pattern for ${ }^{8} \mathrm{~S} \frac{{ }_{7 / 2}}{8} \mathrm{P}_{5 / 2}$ is given in Fig. $2 .{ }^{12}$

Figure 3 (Ref. 13) shows the various Zeeman patterns for the Russel-Saunders coupling case with g-value tabulated in Table I. ${ }^{13}$

From Figs. 2 and 3, it is obvious that $\mathrm{ZAA}$ should use those transitions in which the $\pi$ components are shifted from the unperturbed line by the least amount. Also, it is obvious that lines can be found for all elements that can be used for analysis.

We are now in a position to discuss the dependence of $\mathrm{ZAA}$ signal upon the number of atoms in the furnace.

For the general case, the transmitted light intensity is given by

$$
I_{\|}=\left\{\int_{-\infty}^{\infty} I_{\|}^{0}(v)\left[e^{-\left(\frac{w_{\|}}{\alpha}\right)^{2}} e^{-x k_{v}}\right] d v\right\} e^{-x k}
$$

and

$$
I_{\perp}=\left\{\int_{-\infty}^{\infty} I_{\perp}^{\circ}(v)\left[e^{-\left(\frac{w_{1}}{\alpha}\right)^{2}} e^{-x k_{\nu}}\right] d v\right\} e^{-x k s}
$$

where $\mathrm{ek}_{\mathrm{S}}$ is attenuation of the light by smoke which is independent of $\nu$ over the spectral line profile 


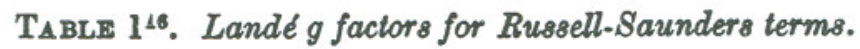

\begin{tabular}{|c|c|c|c|c|c|c|c|}
\hline \multirow{3}{*}{$\frac{S}{\frac{1}{2}}$} & \multirow{3}{*}{$\begin{array}{c}J \\
L-\frac{1}{2} \\
L+\frac{1}{2}\end{array}$} & \multicolumn{6}{|c|}{$L$} \\
\hline & & \multirow{2}{*}{$\frac{S 0}{-}$} & \multirow{2}{*}{$\begin{array}{c}P 1 \\
\frac{9}{8}=0.667 \\
\frac{4}{3}=1.333\end{array}$} & \multirow{2}{*}{ 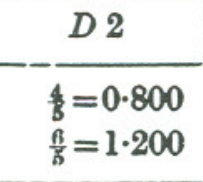 } & $F 3$ & $G 4$ & \multirow{2}{*}{$\begin{array}{c}H 5 \\
\begin{array}{l}11 \\
11\end{array}=0.909 \\
19=1.091\end{array}$} \\
\hline & & & & & $\begin{array}{l}\frac{6}{7}=0.857 \\
\frac{8}{7}=1.143\end{array}$ & $\begin{aligned} 8 & =0.889 \\
\frac{1}{9} & =1 \cdot 111\end{aligned}$ & \\
\hline 1 & $\begin{array}{l}L-1 \\
L \\
L+1\end{array}$ & $\frac{-}{2}$ & $\begin{array}{l}\frac{9}{0} \\
\frac{3}{2}=1.500 \\
\frac{3}{8}=1.500\end{array}$ & $\begin{array}{l}\frac{1}{2}=0 \cdot 500 \\
\frac{7}{8}=1 \cdot 167 \\
\frac{4}{3}=1 \cdot 333\end{array}$ & $\begin{aligned} \frac{8}{3} & =0.667 \\
13 & =1.083 \\
13 & =1.250\end{aligned}$ & $\begin{aligned} \frac{8}{8} & =0.750 \\
2 f & =1.050 \\
2 \delta & =1.200\end{aligned}$ & $\begin{aligned} 4 & =0.800 \\
3 & =1 \cdot 033 \\
3 & =1 \cdot 167 \\
\frac{7}{6} & =1 \cdot 167\end{aligned}$ \\
\hline$\frac{3}{2}$ & $\begin{array}{l}L-\frac{3}{2} \\
L-\frac{1}{2} \\
L+\frac{1}{2} \\
L+\frac{3}{2}\end{array}$ & $\frac{-}{-}$ & $\begin{array}{rl} & - \\
\frac{8}{3} & =2 \cdot 667 \\
26 & =1 \cdot 733 \\
18 & 8 \\
8 & =1 \cdot 600 \\
5\end{array}$ & $\begin{aligned} & 0 \\
& 8=1.200 \\
& \frac{6}{5}=1 \cdot 371 \\
& \frac{4}{5} 50=1.429\end{aligned}$ & 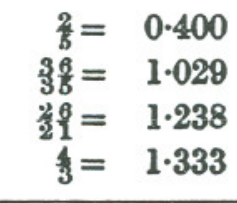 & $\begin{aligned} \frac{1}{7} & =0.571 \\
\frac{82}{63} & =0.984 \\
116 & =1.172 \\
09 & = \\
14 & =1.273\end{aligned}$ & 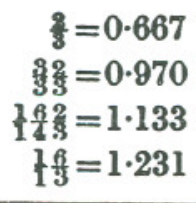 \\
\hline 2 & $\begin{array}{l}L-2 \\
L-1 \\
L \\
L+1 \\
L+2\end{array}$ & $\begin{array}{l}- \\
- \\
- \\
2\end{array}$ & $\begin{array}{c}- \\
-\overline{3} \\
\frac{5}{2}=2 \cdot 500 \\
\frac{1}{6}=1 \cdot 833 \\
\frac{5}{3}=1 \cdot 667\end{array}$ & $\begin{array}{l}\frac{8}{8} \\
\frac{3}{2}=1.500 \\
\frac{2}{3}=1.500 \\
2=1.500 \\
3=2=1.500 \\
3 \underline{3}=1.500\end{array}$ & $\begin{array}{c}0 \\
1 \\
\bar{s}=1 \cdot 250 \\
\frac{4}{z}=1 \cdot 350 \\
\bar{z}=1 \cdot 400\end{array}$ & $\begin{aligned} \frac{1}{3} & =0.333 \\
\frac{1}{1}= & =0.917 \\
\frac{2}{2} & =1 \cdot 150 \\
\frac{2}{0} & =1.267 \\
1, & =1.267 \\
\frac{1}{3} & =1.333\end{aligned}$ & $\begin{aligned} \frac{1}{2} & =0.500 \\
1^{2} 0 & =0.900 \\
17 & =1 \cdot 100 \\
1_{3}^{7} & =1 \cdot 214 \\
\frac{9}{7} & =1.286\end{aligned}$ \\
\hline$\frac{5}{2}$ & $\begin{array}{l}L-\frac{3}{2} \\
L-\frac{3}{2} \\
L-\frac{1}{2} \\
L+\frac{1}{2} \\
L+\frac{3}{2} \\
L+\frac{5}{2}\end{array}$ & $\begin{array}{l}- \\
- \\
- \\
-\end{array}$ & $\begin{array}{c}- \\
- \\
12=2.400 \\
y_{5}=28 \\
35=1.886 \\
12=1.714\end{array}$ & 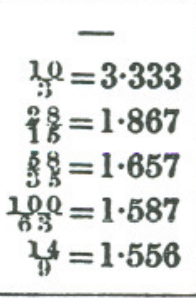 & 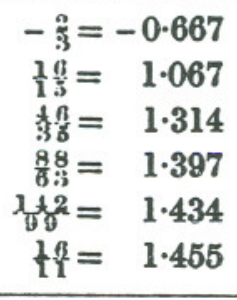 & $\begin{aligned} 0 & \\
\frac{6}{7} & =0.857 \\
8 & =1 \cdot 143 \\
\frac{8}{7} & =1.273 \\
t 1 & =1.343 \\
192 & =1.38 \\
18 & =1.385\end{aligned}$ & $\begin{aligned} \frac{2}{7} & =0.286 \\
\frac{8}{85} & =0.825 \\
306 & =1.071 \\
99 & =1.071 \\
17 & =1.203 \\
30 & =1.282 \\
39 & =1.333 \\
\frac{4}{3} & =1.3\end{aligned}$ \\
\hline 3 & $\begin{array}{l}L-3 \\
L-2 \\
L-1 \\
L \\
L+1 \\
L+2 \\
L+3 \\
\end{array}$ & $\begin{array}{l}- \\
- \\
- \\
- \\
2\end{array}$ & $\begin{array}{c}- \\
- \\
- \\
\overline{7} \\
\frac{7}{3}=2 \cdot 333 \\
\frac{3}{2}=1.917 \\
\frac{7}{4}=1 \cdot 750\end{array}$ & 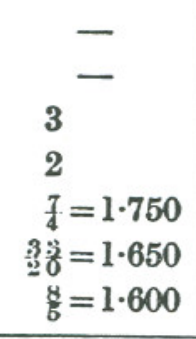 & $\begin{array}{ll}\frac{0}{0} & \\
\frac{3}{2}= & 1 \cdot 500 \\
\frac{3}{2}= & 1 \cdot 500 \\
\frac{3}{2}= & 1 \cdot 500 \\
\frac{3}{2}= & 1 \cdot 500 \\
\frac{3}{3}= & 1 \cdot 500 \\
\frac{3}{2}= & 1.500\end{array}$ & $\begin{array}{rr}-\frac{1}{2}= & -0.500 \\
\frac{5}{6}= & 0.833 \\
\frac{f}{6}= & 1 \cdot 167 \\
\frac{3}{3}= & 1 \cdot 300 \\
\frac{3}{3}= & 1 \cdot 367 \\
\frac{5}{4}= & 1.405 \\
\frac{5}{4}= & =1.429 \\
\frac{1}{6}= & 1.429\end{array}$ & $\begin{aligned} 0 \\
\frac{8}{4}=0 \cdot 750 \\
\frac{27}{2}=1 \cdot 050 \\
\frac{6}{8}=1 \cdot 200 \\
\frac{9}{7}=1 \cdot 286 \\
\frac{75}{8}=1 \cdot 339 \\
\frac{1}{8}=1 \cdot 375\end{aligned}$ \\
\hline
\end{tabular}




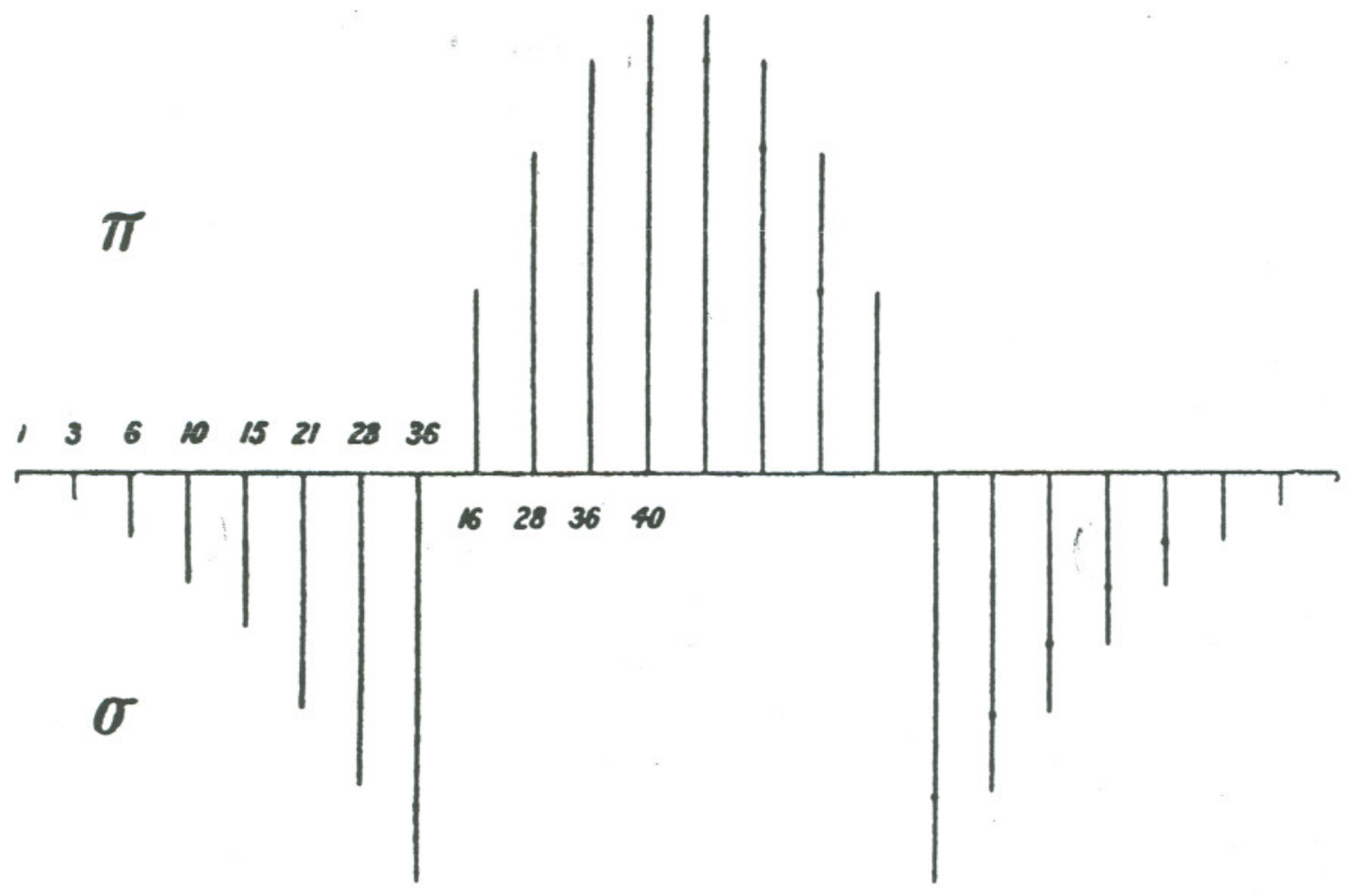

XBL $788-9876$

Fig. 2. Theoretical relative intensities in the ${ }^{8} \mathrm{~S}_{7 / 2} \quad{ }^{6} \mathrm{P}_{5 / 2}$ Zeeman pattern. 
Normal

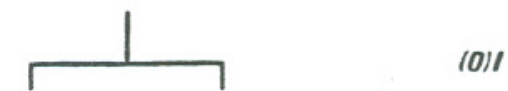

${ }^{3} S \rightarrow{ }^{3} p$
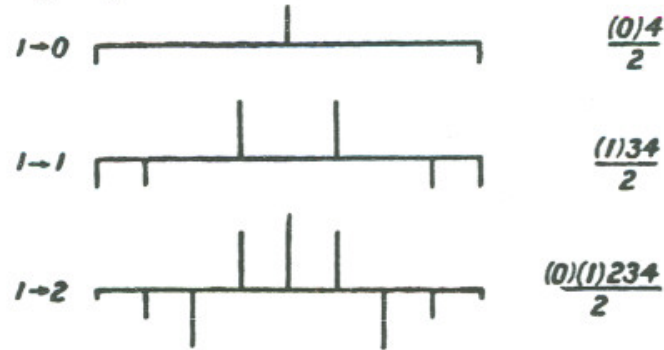

${ }^{3} \boldsymbol{p} \rightarrow{ }^{3} D$

$0 \rightarrow 1$

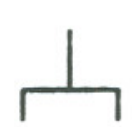

$\frac{(0) 1}{2}$
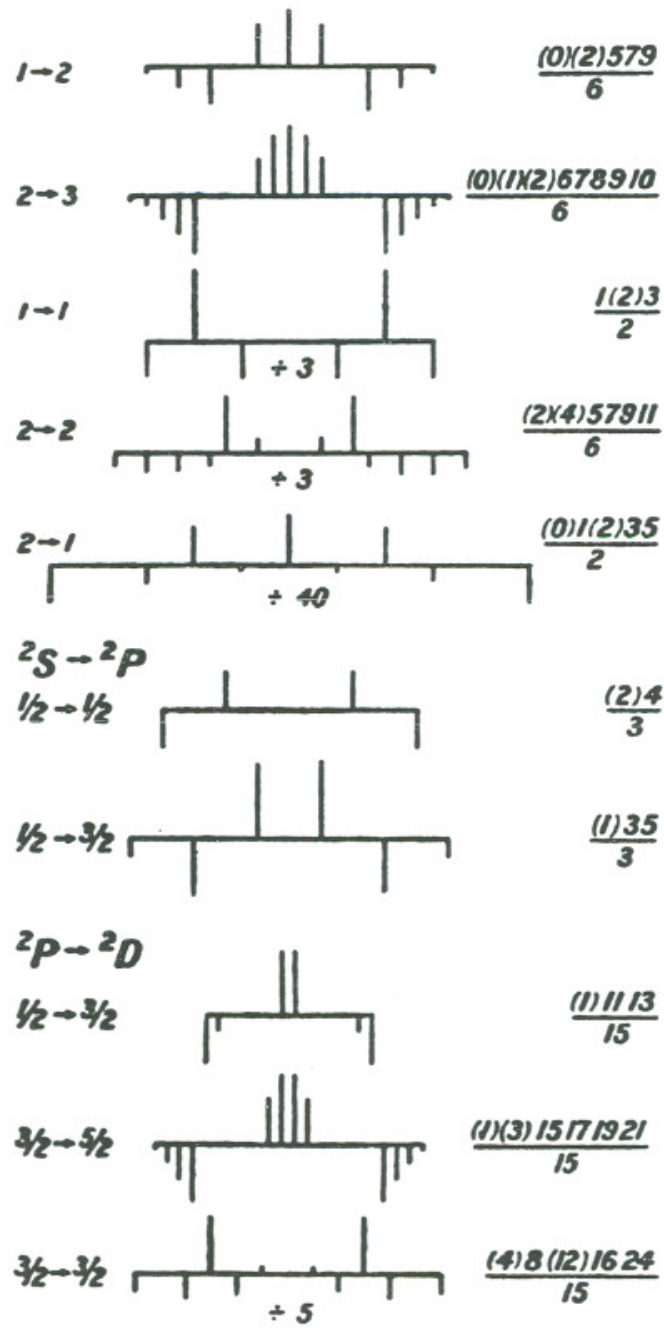

XBL $788-9875$

Fig. 3. Zeeman patterns for several common multiplets. 


$$
\begin{aligned}
& \mathrm{w}_{\|}=\frac{2}{\Delta v_{\mathrm{D}}}\left[v-v_{0}+\sum_{i} \Delta v_{i}(\Delta \mathrm{M}=0)\right] \sqrt{\ln 2} \\
& \mathrm{w}_{\perp}=\frac{2}{\Delta v_{D}}\left[v-v_{O}+\sum_{i} \Delta v_{i}(\Delta \mathrm{M}=0)\right] \sqrt{\ln 2}
\end{aligned}
$$

and

$$
\alpha=\frac{\text { Emission line breadth }}{\text { Absorption line breadth }}
$$

In most cases, we can make the following approximations

$$
\sum_{i} \Delta v_{i}(\Delta M=0)=0
$$

and

$$
\sum_{i} \Delta v_{i}(\Delta M= \pm 1)= \pm \Delta v
$$

where this factor takes into account the shift in ${\sigma^{+}}^{+}$and $\sigma^{-}$Zeeman components. Thus, by writting

$$
I_{\|}=\left\{I_{\|} \int_{-\infty}^{\infty} e^{-\left\{\frac{2\left(\nu-\nu_{0}\right)}{\Delta \nu_{D}} \sqrt{\ln 2}\right\}^{2}} e^{-x k_{\nu}} d v\right\} e^{-x k} s
$$

and

$$
I_{\perp}=\left\{I_{\perp} \int_{-\infty}^{\infty} e^{-\left\{\frac{2\left(\nu-v_{0} \pm \Delta \nu\right)}{\Delta v_{D}} \sqrt{\ln 2}\right\}^{2}} e^{-x k_{\nu}} d \nu\right\} e^{-x k s}
$$


We can show that the attenuation of light by the $\perp$ components is much less than that by the $\|$ component. Let us write

$$
I_{\|}=\left\{I_{\|}^{o} \int_{-\infty}^{\infty} e^{-c\left(\nu-\nu_{0}\right)^{2}} e^{-x k(v)} d \nu\right\} e^{-x k} s
$$

and

$$
I_{\perp}=\left\{I_{\perp}^{0} \int_{-\infty}^{\infty} e^{-c\left(\nu-\nu_{0} \pm \Delta v\right)^{2}} e^{-x k(\nu)} d v\right\} e^{-x k s}
$$

By means of the simple coordinate transformation $v^{\prime}=v \pm \Delta v$, we get

$$
\begin{aligned}
I_{\perp} & =\left\{I_{\perp}^{0} \int_{-\infty}^{\infty} e^{-c\left(v^{\prime}-v_{0}\right)^{2}} e^{-k\left(\nu^{\prime}-\Delta v\right)} d v^{\prime}\right\} e^{-x k s} \\
& =\left\{I_{\perp}^{0} \int_{-\infty}^{\infty} e^{-c\left(\nu^{\prime}-\nu_{0}\right)^{2}} e^{-k\left(\nu^{\prime}-\Delta v\right)} d v^{\prime}\right\} e^{-x k} s
\end{aligned}
$$

since $v^{\prime}$ can be written again as $v$ because the limits of integration are from $-\infty$ to $+\infty$. Now, for $\|$ component, the integral is large only near $v=v_{0}$ and for $\sigma$ component, the integral is large for $v=v_{0} \pm \Delta v$. Therefore, we can write

$$
I_{\|} \simeq I_{\|}^{\circ} e^{-x k\left(v_{0}\right)}\left\{\int_{-\infty}^{\infty} e^{-c\left(v-v_{0}\right)^{2}} d v\right\} e^{-x k} s
$$


and

$$
I_{\perp} \simeq I_{\perp}^{0} e^{-x k\left(\nu_{0} \pm \Delta v\right)}\left\{\int_{-\infty}^{\infty} e^{-c\left(\nu-\nu_{0}\right)^{2}} d v\right\} e^{-x k} s
$$

Thus

$$
\log I_{\|}-\log I_{\perp}=-x\left[k\left(\nu_{0}\right)-k\left(\nu_{0}+\Delta v\right)\right]
$$

and the smoke effect $\mathrm{xk}_{\mathrm{S}}$ is completely eliminated. We shall now show that

$$
k\left(\nu_{0}\right) \ll k\left(\nu_{0} \pm \Delta v\right)
$$

and

$$
\mathrm{k}\left(\nu_{0}\right)-\mathrm{k}\left(\nu_{0} \pm \Delta v\right) \sim \mathrm{N},
$$

the density of atoms in the ground state. A general absorption coefficient which includes Lorentz, Natural and Doppler broadening is given by

$$
\mathrm{k}_{v}=\frac{2 \mathrm{k}_{\mathrm{O}}}{\pi\left(\Delta \nu_{\mathrm{N}}+\Delta \nu_{L}\right)} \int_{-\infty}^{\infty} \frac{\mathrm{e}^{-\left[\frac{2 \delta}{\Delta \nu_{\mathrm{D}}} \sqrt{\ln 2}\right]} \mathrm{d} \delta}{1+\left[\frac{2}{\Delta \nu_{\mathrm{N}}+\Delta \nu_{L}}\left(\begin{array}{c}
\left.v_{0}-\delta\right) \\
]^{2}
\end{array}\right]^{2}\right.}
$$

which can be written as

$$
k_{v}=k_{0} \frac{a^{\prime}}{\pi} \int_{-\infty}^{\infty} \frac{e^{-y^{2}} d y}{a^{\prime^{2}}+(w-y)^{2}}
$$

with 


$$
\begin{aligned}
& w=\frac{2\left(v-v_{O}\right)}{\Delta v_{D}} \sqrt{\ln 2} \\
& a^{\prime}=\frac{\Delta v_{N}+\Delta v_{L}}{\Delta v_{D}} \sqrt{\ln 2}
\end{aligned}
$$

and

$$
y=\frac{2 \delta}{\Delta v_{D}} \sqrt{\ln 2}
$$

For the ZAA atomization furnace operating at one atmosphere, $\Delta \nu_{\mathrm{L}}>\Delta v_{\mathrm{D}}$. For $\left(\Delta v_{L} / \Delta v_{D}\right)=2.4$

$$
\mathrm{k}\left(\nu_{0}\right)=0.25 \mathrm{k}
$$

and

$$
\mathrm{k}\left(\nu_{\mathrm{o}} \pm \Delta\right)=0.08 \mathrm{k}
$$

Thus, by shifting the $\sigma^{ \pm}$components at least by the half-breadth of the absorption line ( $\sim 7$ to $15 \mathrm{~kg}$ for most elements), In $I_{\|}-\ln I_{\perp}$ yields a good measure of the intensity of the absorption line. For a Doppler broadened absorption line

$$
k_{0}=\frac{2 \sqrt{\ln 2}}{\sqrt{\pi \Delta} \nu} \frac{\lambda_{0}^{2}}{8 \pi} \frac{g_{i}}{g_{j}} A_{i j} N_{j}
$$

and to a good approximation $k_{0}^{1}=b k_{0}$ for a pressure broadened absorption coefficient where $\mathrm{b}$ which is $\leqslant 1$ in a proportionality constant. Therefore, $\log I_{\|}-\log I_{\perp} \simeq N_{j}$, the density of the ground state. 
By use of the general expression for $k_{v}$, the signal dependence of $\log I_{\|}-\log I_{\perp}$ as a function of concentration can be determined accurately showing the effect of a range of concentrations. This treatment will show that as the number of atoms increases there will be a linear region, a non-linear region and region where the curve rolls over. Note also, that identical conclusions will be arrived at if the magnetic field is applied to the absorbing elements and linearly polarized resonant light $\|$ and 1 to the magnetic field is used. This is demonstrated in the excellent work carried out by $\mathrm{H}$. Koizumi, et al. ${ }^{15}$ 
IV. Technique of Direct Sample Atomizaton for Determination of Trace Elements

In order to measure trace elements directly from the sample by comparison with a solution standard one must not go through the typical drying, ashing and atomization cycle. Instead the sample must be raised to a high temperature to dissociate metal oxides and high volatility organometallic compounds before the atomic absorption measurement takes place. A typical furnace design used to obtain atomization by this approach regardless of the sample matrix, for the detection of mercury is shown in Fig. 4. In this case, solid or liquid samples are injected into the first expansion chamber that is already held at high temperature to convert the mercury compounds of the samples to atomic mercury. Oxygen is used to minimize smoke from organic compounds.

The furnace is designed in such a way that whether a sample vaporizes slowly or instantaneously, the resulting sample vapor will reach the necessary atomization temperature by the time it enters the absorption tube. A mathematical model was developed to predict the density of atoms in the absorption tube for the slow vaporization case and instant vaporization case and are given by

$$
\frac{\mathrm{N}_{\mathrm{Hg}}(\mathrm{t})}{\mathrm{V}_{\mathrm{a}}}=\frac{\mathrm{N}_{\mathrm{O}}}{\mathrm{V}_{\mathrm{c}}\left(\mathrm{R}-\mathrm{V}_{\mathrm{a}} \mathrm{k}\right)}\left(\mathrm{V}_{\mathrm{a}} \mathrm{Ke} \mathrm{v}^{-\frac{\mathrm{R}}{\mathrm{V}_{\mathrm{a}}} \mathrm{t}}-\mathrm{R}^{-\mathrm{Kt}}+\frac{\mathrm{N}_{\mathrm{O}}}{\mathrm{V}_{\mathrm{c}}}\right)
$$




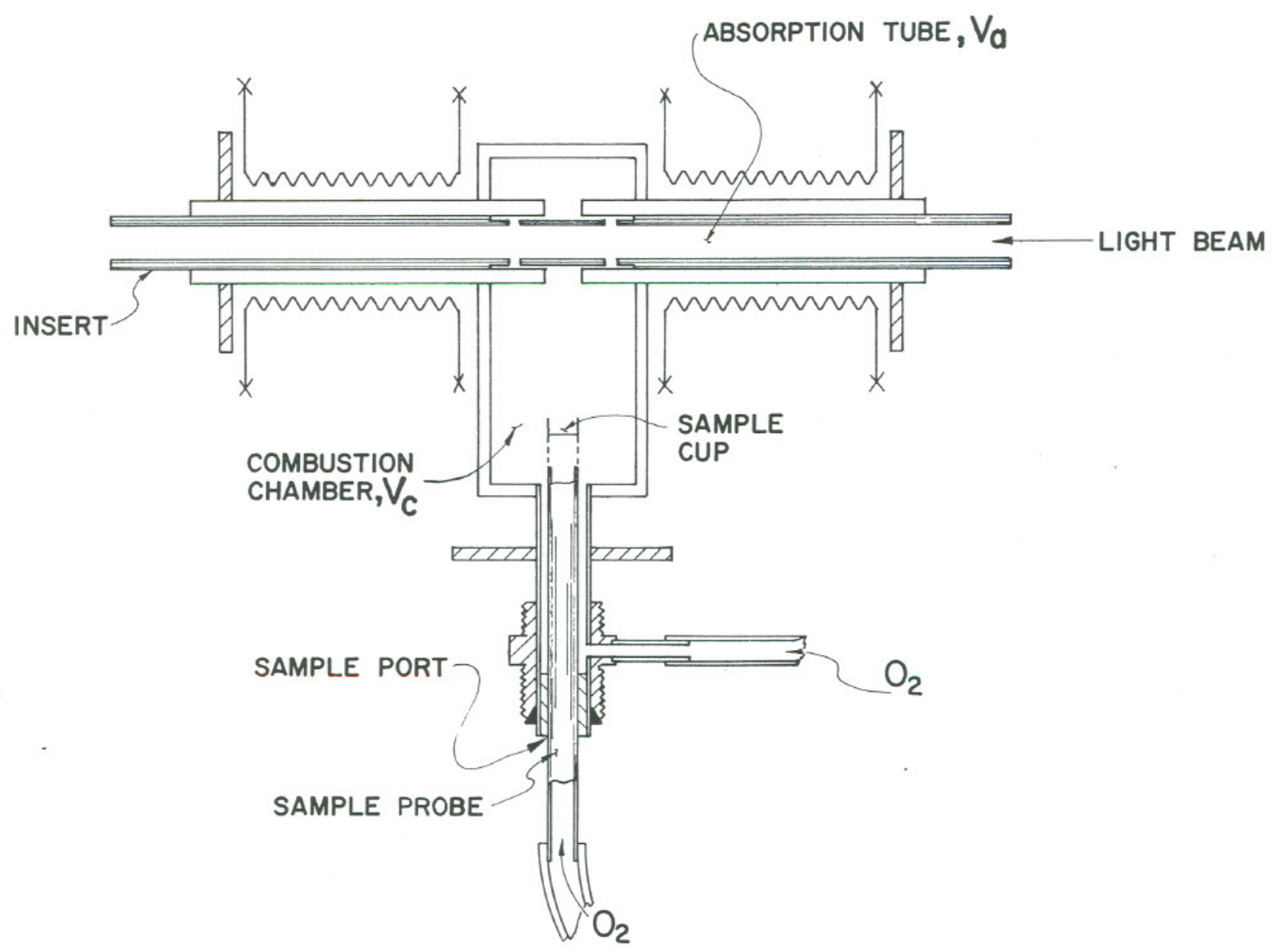

XBL $736-858$

Fig. 4. Dual chamber furnace used for mercury determination. 
and

$$
\frac{\mathrm{N}_{\mathrm{Hg}}(\mathrm{t})}{\mathrm{V}_{\mathrm{a}}}=\frac{\mathrm{N}}{\left(\mathrm{V}_{\mathrm{c}}-\mathrm{V}_{\mathrm{a}}\right)}\left(\mathrm{e}^{-\mathrm{R}_{\mathrm{t}} / \mathrm{V}_{\mathrm{c}}}-\mathrm{e}^{-\mathrm{R}_{\mathrm{t}} / \mathrm{V}_{\mathrm{a}}}\right)
$$

where $\mathrm{V}_{\mathrm{a}}$ and $\mathrm{V}_{\mathrm{c}}$ are respectively the volume of the absorption tube and combustion chamber (lst chamber), $\mathrm{R}$ the carrier gas flow rate, and $\mathrm{k}$ is the rate of evolution of mercury from heating. Excellent agreement between the theoretical prediction and experiments were observed as is shown in Fig. 5. For more refractory elements, a graphite furnace was used to go to higher temperatures. Basically, the same condition as the mercury furnace can be satisfied by using a graphite two chamber furnace.

Figure 6 shows various possible furnace designs to achieve high gas temperature of the sample vapor before entry into the absorption region. Figure $6 \mathrm{~b}$ shows a double chamber furnace cuvette which can be used in commercially available Massmann type furnaces. The same high temperature can be obtained as with the conventional graphite tube cuvette because the surface area of the cuvette is kept small to minimize radiation loss. The recovery of the element in a sample with a complex matrix is highly improved but for some systems (i.e., tetrathyllead in gasoline) it is not complete. Figure $3 c$ shows a closed system furnace. A sample is atomized in a closed space and kept there for a while to achieve complete dissociation. The piston is moved with the thermal expansion of the sample vapor, and just 


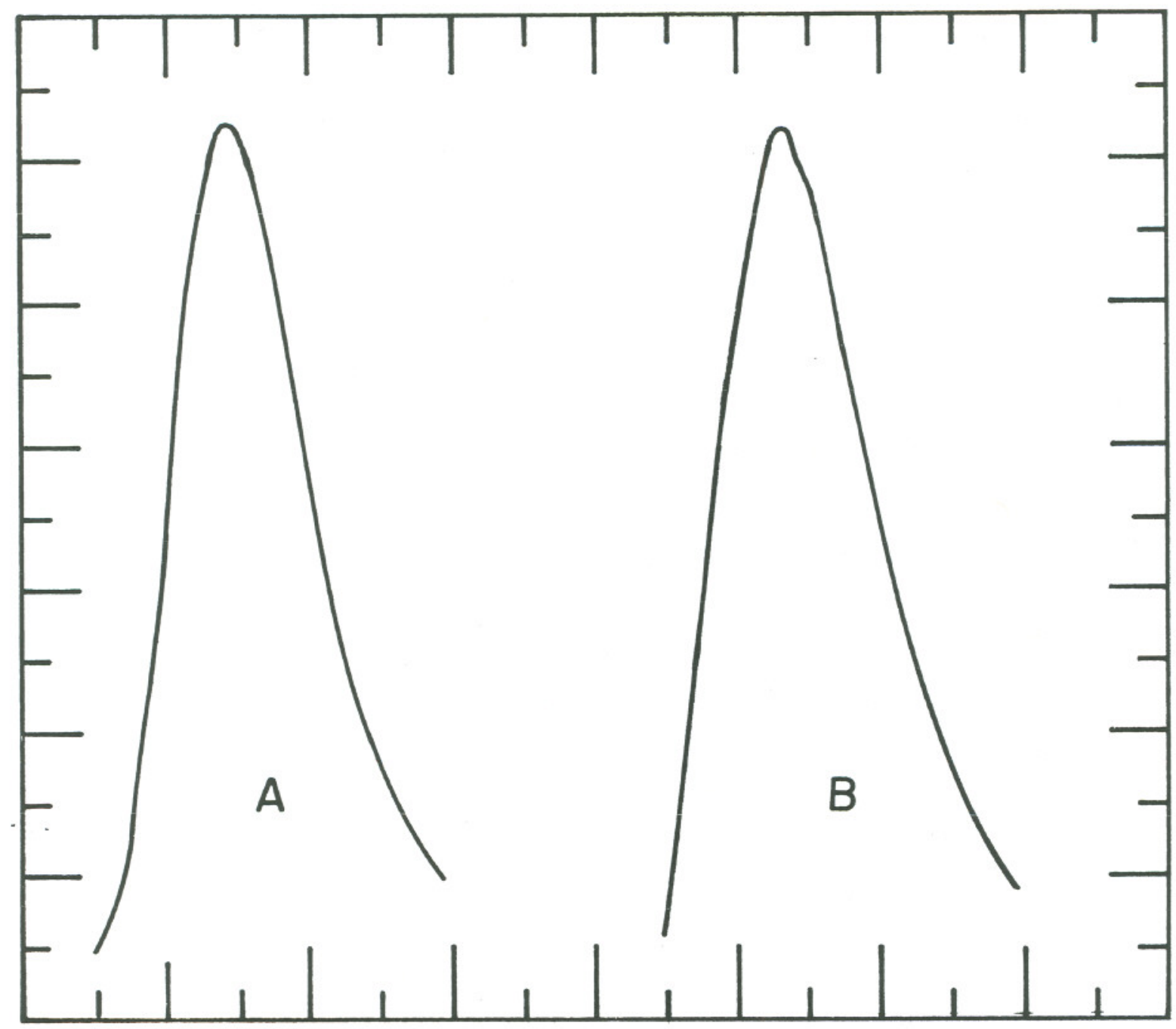

TIME

XBL 7210-1983

Fig. 5. Comparison of experimental and theoretical curves for the density of mercury atoms in the light path as a function of time. 

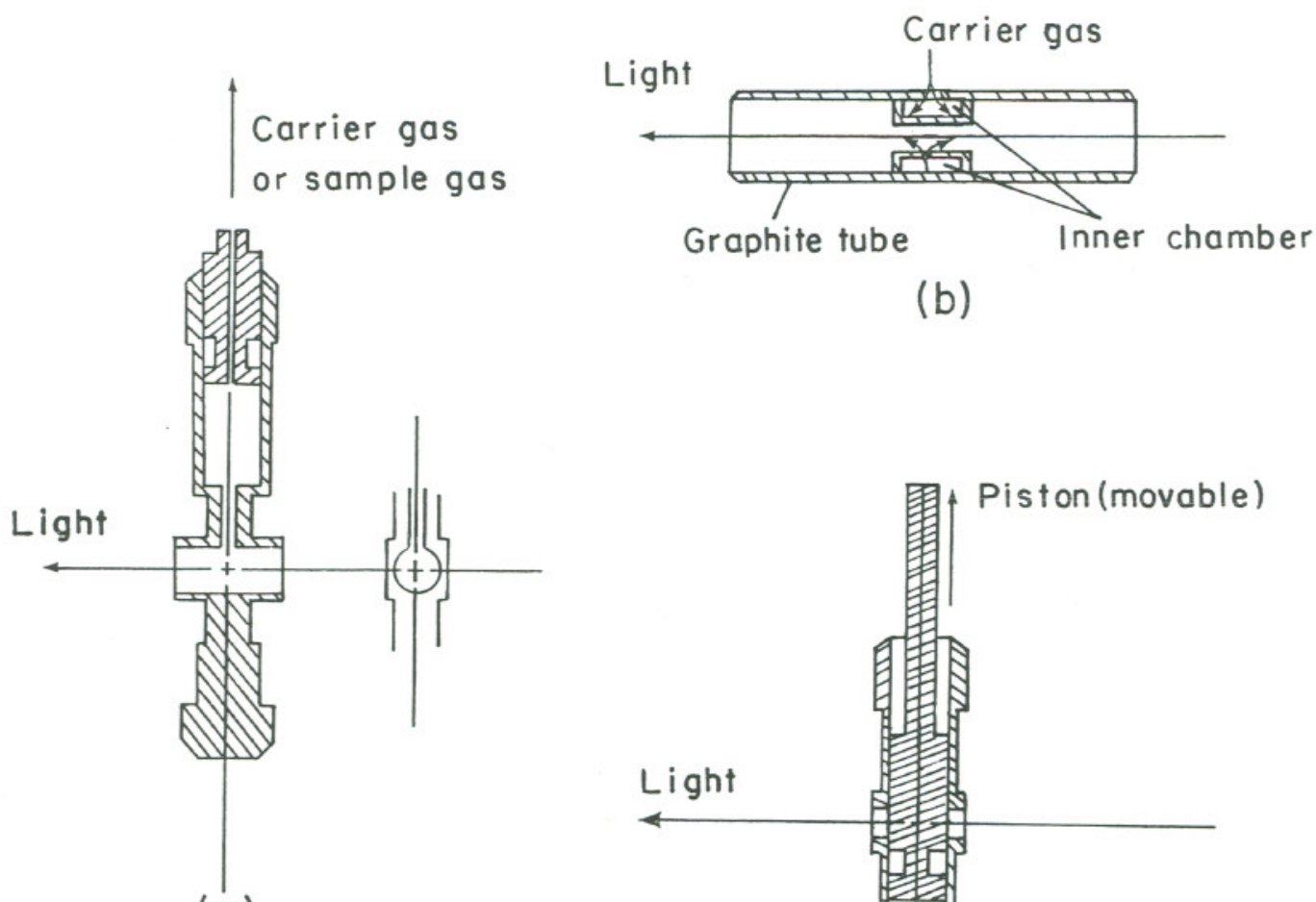

(b)

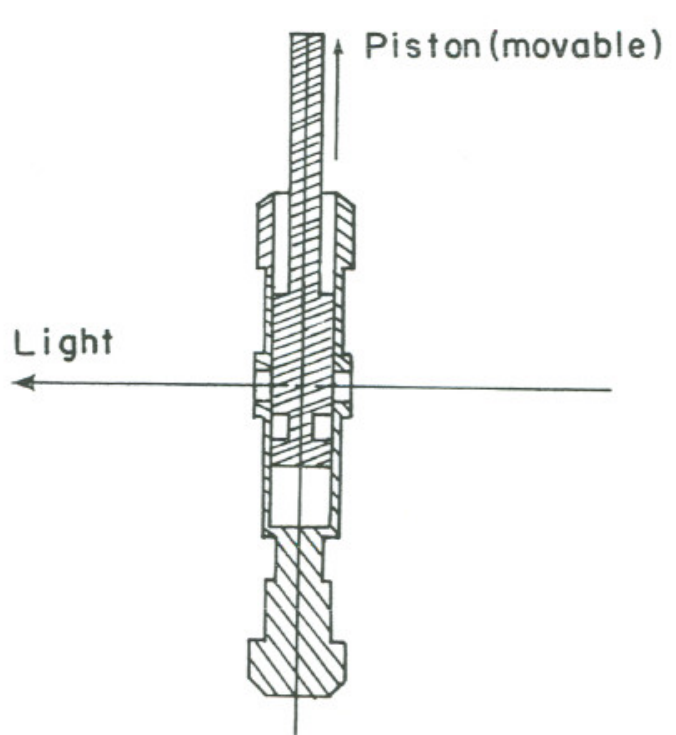

(c)

XBL $787-1213$

Fig. 6. Furnace designs to achieve high gas temperature. 
after the piston passes by the openings an absorption measurement is made. The properties of this cuvette are now being investigated. The circular cavity furnace, ${ }^{16}$ which was recently developed by L'vov, is also a kind of high gas temperature furnace.

Figure 7 depicts the furnace that has given the best results so far. ${ }^{7}$ This furnace was capable of analysing such diverse samples as gasoline (lead present as tetraethyllead), solutions containing $5000 \mathrm{ppm}$ of $\mathrm{MgCl}_{2}$, and crude oil with no previous treatment of the sample. Of course, when the sample matrix is known and it has been experimentally verified that a standard reference value is obtained with direct injection into the Massmann type furnace, the conventional furnace can be used. This is true because of the superior background rejection capability of this instrument compared to conventional atomic absorption spectrometers. 


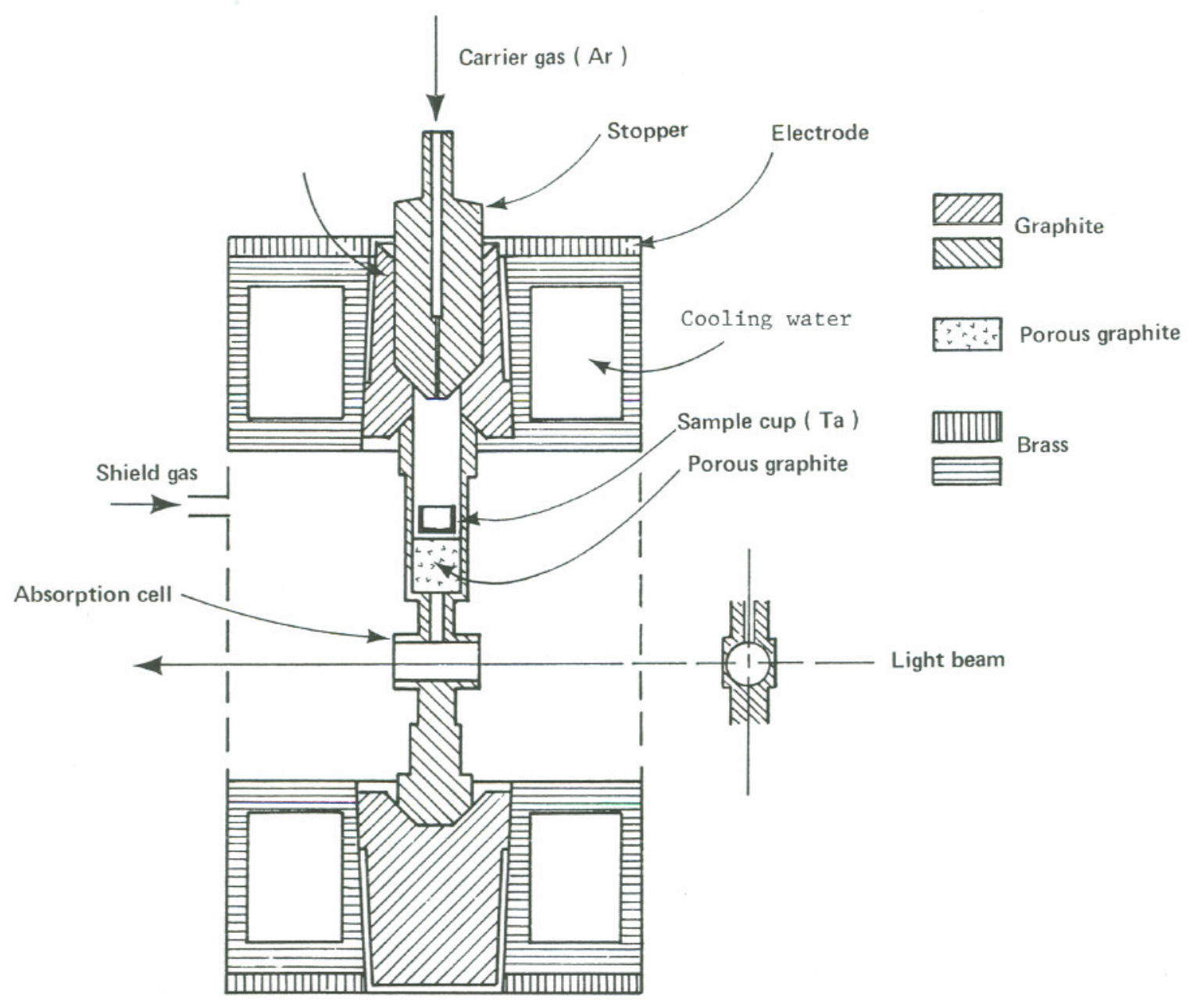

XBL 785-927

Fig. 7. The high gas temperature furnace presently in use. 
V. Magnetically Confined Lamp (MCL)

\section{A. Description}

The MCL is pictured in Fig. 8. It consists of a plasma that is powered by both $\mathrm{a} D \mathrm{DC}$ and RF field at a pressure of between 1 and 10 Torr. The low pressure region is maintained in a stainless steel cross (made of Sagelok fittings) that fits inside a 1 cm gap between the poles of a permanent magnet (field strength of about 10 kgauss). The carrier gas is argon and the vacuum is maintained by interposing a needle valve between the argon tank and a vacuum pump.

The spectrum of most elements can be excited by placing the metal in the cap of the cathode. After the cathode is bolted to the MCL body, the system is evacuated by opening the vacuum pump while the needle valve is closed; after the base pressure of the system is reached the RF power is turned on at full power and the DC power. is increased until the spectrum of the cathode material appears. The plasma tends to be confined to a plane perpendicular to the magnetic field direction. This results because electrons that are moving perpendicular to the magnetic field follow a cycloidal path caused by the interaction of a magnetic force with a moving electric charge, whereas electrons moving parallel to the field do not experience this force. The probability of collisions with gaseous atoms (before completing the transit from the cathode to the anode or wall) is greater for electrons following a cycloidal path than those moving in a straight line. For this reason more excited and ionized atoms (light emitting atoms) are produced in a plane perpendicular to the field. 


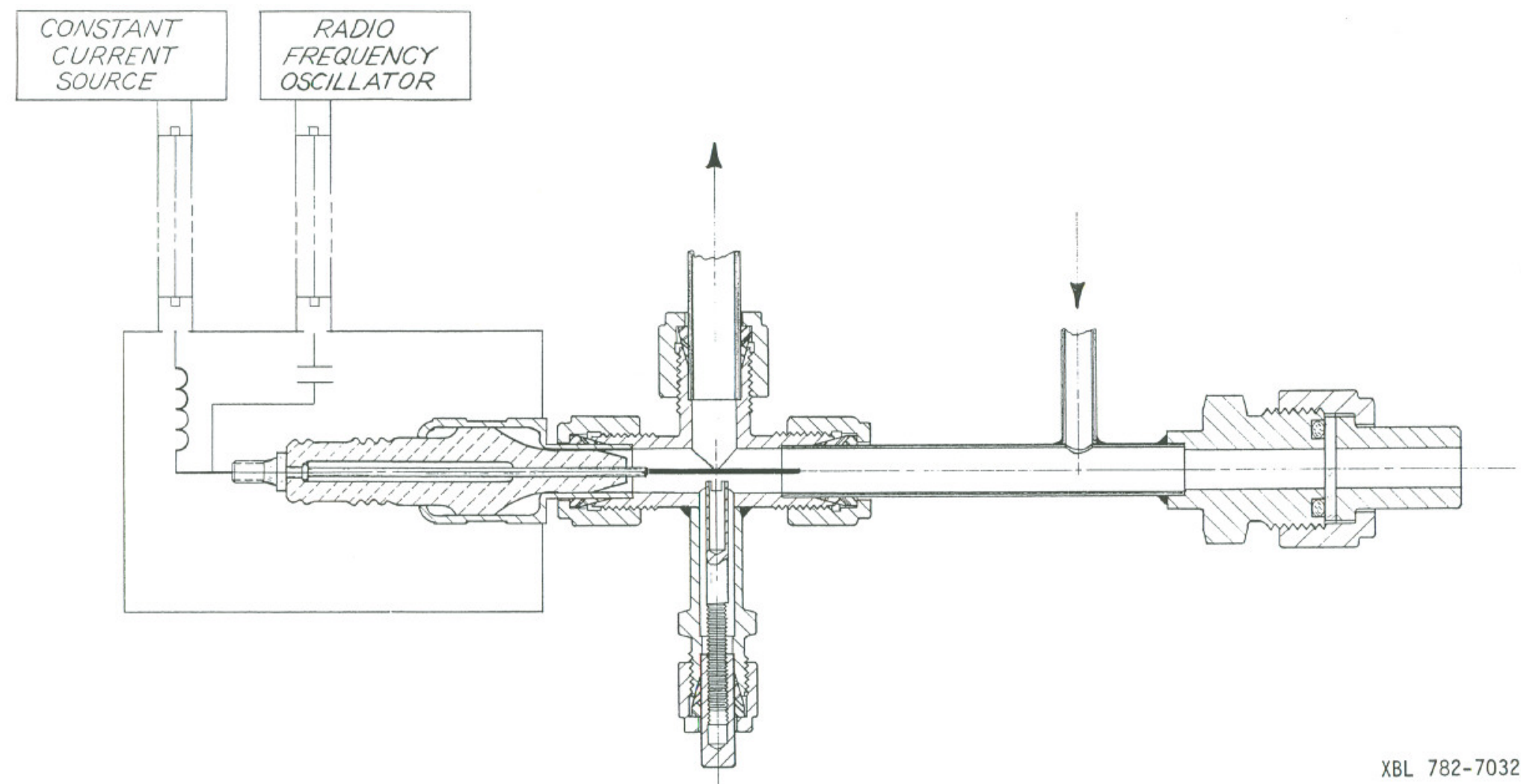

Fig. 8. The magnetically confined lamp. 
As the pressure is increased inside the MCL, the discharge loses its confined planar appearance. This is because at high pressure the mean free path of the electrons becomes so short that the distinction between a cycloidal path and a linear path is lost.

Most cathode materials are introduced into the discharge region by a sputtering process. The more volatile materials (i.e., As, Se, Cd) diffuse into the discharge because of their high vapor pressure. In some cases "thermally assisted" sputtering may be occurring. High intensity spectra of the cathode material can usually be obtained at pressures of 1 to 10 Torr. Good spectra may also be obtained at higher pressures but if it is necessary to use high pressure a dirty or leaky vacuum system may be indicated. The low pressure discharge tends to be more stable than the high pressure. The voltage current measurements demostrate that this is a glow discharge (current 10$100 \mathrm{ma}$, voltage $\sim 200 \mathrm{v}$ ). However an arc discharge (high current and low voltage) may establish itself at high pressures ( $>30$ Torr). In addition, if enough RF power is coupled into the discharge, a unique low DC current, low voltage discharge is obtained (1-100 ma at $\sim 60 \mathrm{v}$ ) In this case the DC power is mainly responsible for exciting the spectrum.

\section{B. Construction}

The importance of a clean vacuum system cannot be overemphasized. It is possible to spend many hours searching for stable MCL operating conditions if the system is dirty or leaky. Running the discharge does clean up the system but the dirtier the system the longer it takes. To minimize these problems keep the lines between the needle valve and MCL, also between the MCL and vacuum pump, as short as possible. 
Use copper lines and Swageloc fittings. Outgassing by heating the copper lines and liquid nitrogen trapping the vacuum pump might also help. If a leak is suspected because of repeated use of the connectors test the system with a helium leak detector. Clean the MCL frequently (daily if volatile materials are used) to remove build up on the anode or body.

The MCL is made by welding a $1 / 4$ inch Swageloc reducer to a $3 / 8$ inch union tee so that a cross results (see Fig. 3) (a 3/8 inch union cross does not fit between the magnetic pole pieces). Before welding, the sides of the tee are milled so that it will fit in a $1 \mathrm{~cm}$ pole gap. The window and gas inlet section are made by holding a quartz (suprasil) window onto a $3 / 8$ inch Cajon vCO 0 -ring vacuum coupling and welding a $1 / 4$ inch tube onto the body of this coupling. This assembly is held onto the MCL body with a $3 / 8$ inch Swageloc connector. The automabile spark plug is machined down so that it can be held onto the MCL body with a $3 / 8$ inch Swageloc fitting. (The electrical continuity of the spark plug should be checked since some will transmit a high voltage spark but not a low voltage DC current.) The $1 / 4$ inch reducer that was welded on the MCL body is used to hold the cathode and the other leg is attached to the vacuum pump through a shut off valve. C. Preparation of Cathode

If the element of interest is available in rod form, the MCL cathode can be simply fabricated by cutting a $3 / 8$ inch diameter rod with a 10-32 thread to such a length that a $1 \mathrm{~mm}$ gap exists between the anode and cathode after they have been mounted in the Swagelok cross. The 10-32 screw can be held in position by screwing into the 
Swagelok end piece and will not be disturbed by attraction of the magnetic feld. $\mathrm{Cr}, \mathrm{Cu}, \mathrm{Fe}$, and $\mathrm{Zn}$ have been excited this way. A stainless steel rod was used for $\mathrm{Fe}$, and $\mathrm{Cr}$ and a brass rod was used for $\mathrm{zn}$. If the metal is available only in powder or pellet form, a cup must be formed in a $3 / 8$ inch stainless steel electrode by drilling a hole parallel to the long axis of the rod. This cup type cathode is also mounted with a $2 \mathrm{~mm}$ electrode gap. The following procedures were used for the elements listed below.

$\underline{\mathrm{Ag}}$

Ag spectrum was obtained by tamping the foil into the cup that resulted when a hole was drilled into a 10-32 stainless steel screw. It is very easy to obtain a quiet steady discharge of this element even with vacuum systems that are not particularly clean.

\section{Al}

Al spectrum was obtained by using a $3 / 8$ inch $A l$ rod as the cathode. This material forms a very stable oxide that interferes with the sputtering process. To overcome this tendency the discharge is $r$ un at high DC power $(100-200 \mathrm{ma}$ at $\sim 200 \mathrm{v})$ so that enough $\mathrm{Al}$ vapor is produced to react with any source of oxygen or water in the system (i.e., outgassing of walls and lines) and still leave some vapor that can be excited to give off spectrum. System cleanliness and tightness from leaks is especially important for this element.

\section{$\underline{\text { As }}$}

As spectrum was obtained by drilling a $1 / 2$ inch deep hole in a 10-32 stainless steel screw. (The threads of the upper $1 / 2$ inch length had been turned off on a lathe.) As metal powder was tamped 
into the lower half of the hole. Copper turnings (J. T. Baker and Co.) were hammered on top of the As until the hole was filled. Because of the high vapor pressure of this material the D.C. power should be kept low $(20-100 \mathrm{ma}$ at $\sim 200 \mathrm{v})$. In fact it is believed the main function of the $\mathrm{Cu}$ is to provide a thermal mass such that the vapor pressure will be constant. The appearance of the cathode after running suggests that a Cu As alloy has formed. This alloy may also act to help deliver the As into the plasma at a constant rate. If the discharge is unstable after the initial set up, this may be due to outgassing of $\mathrm{As}$ and $\mathrm{Cu}$ in the cup. In this case $\mathrm{r}$ un at higher power for about 5 minutes to hasten the process, then lower the power for routine operation. $\mathrm{P}$ and Se cathodes are prepared in the same way.

$\underline{\text { Au }}$

Au spectrum was obtained by tamping the foil into the cup that resulted when a hole was drilled into a 10-32 stainless steel screw (the threads had been cut off with a lathe). It is very easy to obtain a quiet steady discharge of this element even in a vacuum system that is not particularly clean.

$\underline{B}$

B spectrum was obtained by placing a sliver of the metal in the cathode cup such that the edge of the metal extended about $2 \mathrm{~mm}$ above the electrode. The distance between the edge of the $\mathrm{B}$ and the anode was also $2 \mathrm{~mm}$. Although this worked well, an attempt to obtain the spectrum by filling the cathode cup with the power was unsuccessful. The improvement obtained by this arrangement may be due to "thermally assisted" sputtering, the electric field distortion caused by a sharp 
edge on the cathode, or both. Other metals that showed improvement from a similar approach were $\mathrm{Si}, \mathrm{Ni}$ and $\mathrm{Sb}$.

$\underline{\mathrm{Ca}}$

The calcium spectrum was obtained by placing a Ca-Ag alloy into the cathode. This was prepared by heating Ca metal in a stream of hydrogen to remove the oxide coating. The Ca was on top of a Ag sheet in a quartz tube. After the hydrogen treatment the gas stream was changed to argon and the heat increased until the $\mathrm{Ca}$ and $\mathrm{Ag}$ flowed together in a molten mass whose composition was about 50:50. A portion of this material was placed in the cathode and a steady Ca discharge was obtained.

The discharge was very unstable if $\mathrm{Ca}$ metal was used directly. This probably resulted from the oxide coating or the metal. After running the discharge Ca was deposited on the body of the MCL. and anode. This must be cleaned off before attempting to excite another element since the presence of $\mathrm{Ca}$ in the discharge tends to lower the electron temperature.

$\underline{\mathrm{Cd}}$

The Cd spectrum was excited from a stainless steel cathode with a hole drilled to form a cup. Metal shavings were tamped into this hole. Cd is a very easy element to excite but the intensity is difficult to control. This probably results because of the high vapor pressure at the cathode temperature combined with the large oscillator strength of the Cd $228.8 \mathrm{~mm}$ transition. Stabilize the discharge by operating in a very clean vacuum system and check for drift by periodically running standards. 


\section{$\underline{\mathrm{Cr}}$}

The MCL was made from $\mathrm{Cr}$ power placed in a stainless steel cup cathode. Dischrge was run at $\sim 300$ microns, (as measured by the gage at the vacuum pump) $400 \mathrm{v}$, and $150 \mathrm{ma}$. Either the $359.3 \mathrm{~mm}$ or $357.9 \mathrm{~nm}$ may be used after discharge is established. Slowly reduce RF power until it is finally off. A blue coloration should occur above cathode. If MCL is run at low pressure $(100 \mu)$, the $358.8 \mathrm{~mm}$ Ar line will be mistaken for the $\mathrm{Cr}$ line and the $\mathrm{Cr}$ sensitivity will be poor or nonexistent.

Generally lamps must be $r$ un at high power a few minutes for clean up before a good $\mathrm{Cr}$ line can be obtained. Do not run the MCL at greater than a $400 \mathrm{~V}$ setting on the power supply.

\section{Cuㅡ}

The Cu spectrum was excited by using a $3 / 8$ inch $\mathrm{Cu}$ rod that had 10-32 threads on one end. The spectrum is very easy to obtain.

$\underline{F e}$

The Fe spectrum was excited from a $3 / 8$ inch $10-32$ stainless screw. This cathode had the upper threads machined off and a hole drilled into the upper section so tht it could also be used for other materials as well.

Ga

Ga spectrum was excited by tamping Ga chips in a cup of a stainless steel cathode (made from 10-32 screw).

$\underline{\mathrm{Hg}}$

The Hg light source is an electrodeless discharge tube (EDL) that is excited using only the RF power supply. It fits into a coil 
that couples the RF power into the EDL. This coil is part of an assembly that contains a brass block that fits between the pole pieces of the magnet. It is important to cool the EDL, using the hole in the block, while running the discharge. These tubes last for several months of continuous operation.

$\underline{\text { In }}$

In spectrum was made by tamping In strips into the cup of a stainless steel cathode (made fram a 10-32 screw).

$\underline{\mathrm{Na}}$

Na spectrum was excited by placing the metal in the cup of a stainless steel electrode (made from a 10-32 screw). After discharging the metal had coated the anode and the body of the MCL. Because of this coating it was difficult to excite other elements in this MCL. This results because the ionization potential of $\mathrm{Na}$ is much lower than most other elements. The presence of $\mathrm{Na}$ from the walls lowers the average electron temperature of the discharge so that the probability of excitation of other elements is reduced. Because of this effect extra care should be taken to clean out the MCL after exciting Na.

$\underline{\mathrm{Ni}}$

The $\mathrm{Ni}$ spectrum was obtained by spot welding a $\mathrm{Ni}$ sheet to the top of a stainless steel cathode. See the description for preparation of B cathode for an explanation of why that was necessary.

$\underline{\mathbf{P}}$

This spectrum was excited in the same way as the As spectrum. See description under As for details. 
$\underline{\mathrm{Pb}}$

$\mathrm{Pb}$ spectrum was excited by tamping the metal (powder or shot) into the cup of a stainless steel cathode (made from 10-32 screw).

$\underline{\mathrm{Pt}}$

This spectrum was excited by tamping the foil into the cup of a stainless steel cathode (made from 10-32 screw).

$\underline{\mathrm{Sb}}$

The spectrum of $\mathrm{Sb}$ was excited both by tamping the powder into the cathode cup and by mounting a metal chunk so that it projected above the cathode. The metal chunk gave better results since the intensity kept fading when the powder was used.

Se

This spectrum was excited in the same way as the As spectrum. See description under As for details.

$\underline{\text { Si }}$

This spectrum was excited in the same way as the B spectrum. See description under B for details.

$\underline{\mathrm{Zn}}$

The $\mathrm{Zn}$ spectrum was excited by making the cathode out of a brass rod $(\sim 708 \mathrm{Cu}, \sim 308 \mathrm{zn})$. This was done by machining 10-32 threads on a $3 / 8$ inch rod.

As is the case in atomic absorption analysis, the shape of the analytical curve (and hence the sensitivity) is affected by the amount of self reversal of the resonate line. (Self reversal refers to the absorption of the center of the analytical line by atoms in the ground state that are between the emitting atom and the detector). In most 
cases this effect is so small that it can be ignored. However if a volatile element with a high oscillator strength is excited (i.e., Cd or $\mathrm{Zn}$ ) periodic checks should be made to see if changing amounts of self reversal are changing the sensitivity. This is done by noting if a variation occurs in the signal intensity when the same standard is periodically run during the time required to obtain the signals from samples. If this does occur the data obtained from the standard allows the approprite corrections to be made.

When the discharge is first initiated the spectrum of argon or the stainless steel electrode may dominate. Confusion may result if the analyte emission line and stainless steel lines are both passed by the monochromater. So far, this has only occurred when the $\mathrm{Pb}$ 283. $3 \mathrm{~nm}$ line was used. The problem was solved by running the DC power high enough so that $\mathrm{Pb}$ lines dominated the spectrum. 
VI. Sensitivity

The ultimate measure of performance of the ZAA instrument is its lower limit of detection. The lower level of detection value that appears in Table 1 is the amount of metal producing absorption equal to twice the background fluctuation. This quantity is determined by the noise in the light source, noise in the electronics and is affected by the optical alignment.

For comparison the quantity of metal required to produce 18 absorption with atomic absorption instrumentation is also tabulated. ${ }^{17}$ These values were chosen because they were obtained from a furnace that closely resembles the one used in the ZAA work.

It should be pointed out that the definition of sensitivity that is recommended by the International Union of Pure and Applied Chemistry differs fram the quantitites defined above. ${ }^{18}$ However these measures of sensitvity are in common use in the field of atomic absorption spectroscopy. 
Table I. Comparison of ZAA and AA Sensitivity

\begin{tabular}{|c|c|c|}
\hline Element & $\begin{array}{c}\text { ZAA } \\
\text { LLD (pg) } \\
\end{array}$ & $\begin{array}{c}\text { AA } \\
\text { Sensitivity } \\
\end{array}$ \\
\hline $\mathrm{Ag}$ & 5 & 8 \\
\hline As & 100 & 160 \\
\hline $\mathrm{Au}$ & 20 & 60 \\
\hline B & 1,000 & -- \\
\hline $\mathrm{Ca}$ & 20 & 3 \\
\hline $\mathrm{Cd}$ & 10 & 2 \\
\hline $\mathrm{Cu}$ & 5 & 70 \\
\hline $\mathrm{Cr}$ & 100 & 30 \\
\hline $\mathrm{Fe}$ & 30 & 50 \\
\hline $\mathrm{Ga}$ & 50 & 1,200 \\
\hline $\mathrm{Hg}$ & 100 & 15,000 \\
\hline In & 500 & --- \\
\hline $\mathrm{Ni}$ & 300 & 200 \\
\hline $\mathrm{Pb}$ & 30 & 80 \\
\hline Pt & 1,500 & 750 \\
\hline $\mathrm{Sb}$ & 400 & 500 \\
\hline $\mathrm{Se}$ & 300 & 3,000 \\
\hline $\mathrm{Zn}$ & 7 & 0.7 \\
\hline
\end{tabular}


VII. Sample Manipulation

A. Introduction

In this section procedures for sample manipulation will be described that have particular application to the Zeeman Atomic Absorption Method. Before going into specifics, however, there are certain general aspects of chemical analysis that should be emphasized.

1) Sampling

In almost every case the sample presented to the analyst is of value only because it provides information about a larger collection of material. If the sampling procedure is such that this condition is not valid then the results of the analysis are of no value. It is the responsibility of the analyst to determine if the results will be of value before running the sample. Sampling procedures are discussed elsewhere. ${ }^{19-21}$

\section{2) Testing the analytical method}

There are many pitfalls in any analytical procedure that will result in an incorrect analysis. These can occur during the operations that precede insertion of the sample into the instrument (i.e., grinding, dilution, concentration, ashing, transfer) as well as at later stages in the analysis (i.e., changing instrument sensitivty, incorrect standard, molecular formation).

There are four procedures that will minimize the possibility that the results will be subject to these pitfalls: 
a) analyze a known sample.

b) have the sample analyzed by different techniques (i.e., NAA, XRF) .

c) add a known amount of analyte and determine the recovery.

d) utilize the standard additions method. Samples of known composition can be obtained from the National Bureau of Standards ${ }^{22}$ and are referred to as standard Reference Materials (SRM). The known sample should be as similar as possible to the unknown. Fortunately the Bureau of Standards has a large variety of sample types, i.e., coal, coal fly ash, bovine liver, orchard leaves, oil and trace elements in water. If analyses of the known sample gives the correct result the pitfalls described above have been avoided (provided the analyte is in the same form in the unknown) .

If the recovery of a known amount of analyte is to be determined care must be exercised to see that the analyte added is as much as possible identical with that in the sample. This presents few problems if aqueous solutions are being analyzed. However if oils, for example, are being analyzed directly, and it is believed that the metals exist as soluble metal organics in the oil, then metal organics should be added to the oil in order to determine the recovery. The same approach should be followed if the standard additions 
method is used. ${ }^{23}$ oil soluble metal organics can be obtained fram a number of sources. 24

The Zeeman atomic absorption (ZAA) method represents the best technique that has yet been devised for background correction. It has long been recognized that background interferences are the most serious cause of error in the atomic absorption technique. ZAA has greatly lessened this source of error. However other sources of error still remain. Since the essence of the technique is to compare the signal from a sample with that from a standard, any interaction that causes there to be a difference betwee these two signals (when the same amount of analyte is present) is a source of error. A description of most of these sources of error and how to correct for them is given in what follows. However, it may be necessary to carry out the procedures described above in order to discover that a problem exists.

\section{B. Matrix Modification}

It is the presence of the sample matrix that ultimately limits $A A$ or ZAA analysis. Even if the matrix is very pure water a sensitivity limit is imposed by the amount of water that is possible to transfer to the furnace. The more common limitation is imposed because of light scattering and absorption by the volatilizaton of salts or organic matter in the sample. This has led to the practice of dissolving the sample to destroy the organic matter or solvent extraction to separate the analyte from the unwanted salts. A summary of these techniques is presented by Kirkbright and Sargent. ${ }^{23}$

Because of the superior background correction capabilities of the ZAA instrument, this section will describe methods that do 
not require such extensive matrix modification. Minimizing sample manipulation minimizes possibilities of sample contamination, loss of analyte and other errors that result from the carrying out of a complex series of operations. Also, less demand is made upon the time of the chemist. However, some matrix modification procedures were utilized that required a minimum of manipulation.

In some cases the sample had to be diluted so that quantities of analyte that were within the working range of the instrument could be introduced into the furnace. A graphite powder formulated for use in diluting emission spectrographic samples was used for solid samples (Ultra U.C.P.-2 325 mesh). 25 A Wig-L-Bug mixer was used to blend the sample with the graphite. In most cases blank runs indicated no detectable signal from the analyte contained in the amount of graphite used in running the sample $(\sim 10 \mathrm{mg})$. However, a small Cu signal was obtained. This could be removed by heating the graphite diluent in the Massmann furnace before using. Other workers have also used this technique. 26

Sample dilution was also required with some shale oil samples. In this case methyl isobutyl ketone (MIBK) or xylene was used as a diluent. To avoid pipetting errors with the viscous liquids, weight measures were made of the container before and after transfer of the oil for dilution. It was also necessary to monitor the change in weight after dilution in order to correct for the evaporation of the solvent during the time the analysis was carried out.

Matrix modification was also effected by the addition of a $508 \mathrm{NH}_{4} \mathrm{NO}_{3}$ solution to salt water samples and a $2 \% \mathrm{Ni}\left(\mathrm{NO}_{3}\right)_{2}$ solution 
to samples for the determination of arsenic. ${ }^{27}$ (The $\mathrm{NH}_{4} \mathrm{NO}_{3}$ causes $\mathrm{NaCl}$ to volatilize at a low temperature by the formation of $\mathrm{NH}_{4} \mathrm{Cl}$ and $\mathrm{NaNO}_{3}$. The $\mathrm{Ni}$ prevents loss of arsenic during sample charring by the formation of the refractory nickel arsenide.) However, it was found that the effect of these additives differed depending upon the sample matrix and recovery experiments should be carried out to determine their usefulness.

\section{Furnace Effects}

There are a number of variations in the signal that are caused by interactions of the analyte with the furnace. The analyst should be aware of these effects in order to minimize time spent in running samples.

The furnace heating element becomes more porous as it is

used. This occurs because small channels are formed because of reaction of the carbon with the sample (or oxygen that has diffused into the furnace) to produce $\infty$. Evidence of this effect can be seen by placing a drop of water on a new graphite tube and also on a tube that has been used many times. The drop will sit as a hemisphere on the new tube but will be rapidly sucked into the old tube. If the solution is evaporated to dryness the residue will be on the surface in the first case and will be embedded inside the tube walls in the second case. This results in a difference in signal because in one case the analyte is volatilized off of the surface and in the other case it must diffuse through the walls. Correction for this effect can be made by running frequent standards and replacing tubes periodically. 
The tubes will deteriorate much faster if salty samples are run or if a high temperature is needed to volatilize the analyte. If large volumes of solutions are used deterioration is hastened because water condenses on the furnace walls during the drying cycle. When the tube temperature is increased this water vaporizes and contacts the tube while it is at a high temperature. This causes $C O$ to be produced because of the reaction of the water and carbon at high temperature. This problem can be lessened by using low temperatures and high gas flow rates during drying.

Differences in porosity have also been found to effect the signal obtained from MIBK solutions. In this case the aqueuous standards gave a larger signal than standards prepared in MIBK solutions if the tube were porous. This resulted because the MIBK penetrates the tube to a greater extent. No such effect was observed if a new graphtie tube were used.

Furnace contamination can also occur. This will result if samples containing large quantities of salts are volatilized. Some of the salts settle as powders on the furnace wall. Although the analyte will be there in small quantity there may be large quantities of other salts. If later other samples are analyzed where the analyte is the same element as existed in large quantity in the previous sample, contamination may result. This occurs because the powder is knocked off the wall as the sample is inserted. Generally the signal will vary greatly from $r$ un to $r$ un if this is the problem. This contamination can be removed by disassembly of the furnace followed by a thorough scrubbing. Contamination can also result from the sample transfer spoon 
if solid samples are being analyzed. To distinguish between these two sources simply tap the outside of the furnace several times, then raise the temperature to see if a signal is obtained. No signal indicates contamination from the transfer spoon.

Two modifications were made in the furnace tube that were valuable aids to the analysis. As originally designed, the hole in the tube that was intended for injection of the pipette tip was too small to allow the tip to reach the side of the tube opposite the hole. This led to lack of reproducibility because at times a droplet of liquid would remain on the tip and not get transferred. This problem was solved by slightly enlarging the transfer hole such that the pipette tip rested against the side of the tube opposite the hole. This resulted in more reproducible transfer of liquids since most of the liquid rolled to the bottom of the tube upon ejection and the small amount of liquid that had formally hung up on the tip now was transferred to the tube wall.

When making arsenic determinations it was noticed that a sensitivity increase of about 5 was obtained by drilling 4 large holes $\left(1 / 8^{n-3 / 16 ")}\right.$ in both ends of the graphite tube. This sensitivity increase occurs because the gas flowing through the ends of the tube does not push the As vapor out so rapidly. Work by L'vov ${ }^{28}$ indicates that this configuration will also lessen scattering of light by salt vapor. However, no measures were made of this effect. Blackbody emission from the furnace can have an effect on the signal obtained. This results because of the characteristics of the logrithmic amplifier. This device converts linear input currents 
( $i_{i n}$ ) to output currents ( $i_{\text {out }}$ ) according to the following relationship

$$
i_{\text {out }}=\operatorname{klog}_{10} i_{\text {in }} / i_{\text {ref }}
$$

where $\mathrm{K}=\mathrm{a}$ constant and $\mathrm{i}_{\text {ref }}=$ an internal reference current. The ZAA instrument generates an AC signal that depends on the intensity of the $\pi$ and $\sigma$ component that rides on top of the DC signal from the light source. If the intensity of the DC signal increases the value of the ratio $\left(i_{\sigma}-i_{\pi}\right) / i_{\text {ref }}$ will decrease since the difference between the DC current and $i_{\text {ref }}$ becomes larger.

This effect is more serious at high wavelengths because of the shape of the blackbody curve. It is also dependent on the slit width of the monochromater and the amount of stray light passed by the monochromator. Because of the high intensity of the MCL light source, change in DC level is rarely a problem. (It takes much more furnace light to have an appreciable effect when the PMr is kept at the low voltage used with these light sources.) If this effect occurs optimize the alignment of the furnace and increase MCL intensity.

One other furnace effect should be mentioned. This applies only to the dual chamber furnace used for mercury. It was noted when running the standard reference material coal sample that low results were obtained if the sample weight exceeded $24 \mathrm{mg}$. It had already been observed that the signal size depends upon the oxygen flow rate through the furnace. If gas evolved by the sample is sufficient to increase the flow rates while the mercury is moving through the furnace a low result will be obtained because of the same effect. The presence 
of this effect can be detected by comparing Hg values obtained from large sample weights with those obtained from small sample weights.

D. Experience with Specific Elements

Because of the superior background correction capability of the ZAA instrument, the possibility of sample analysis without first going through a dissolution step is attractive. The results of direct determination of specific elements in a variety of sample matrices are presented below.

Solid samples were tared in a transfer spoon made by holding a pipette tip to a stainless steel wire with epoxy cement. A hole was drilled through a brass bar such that the snug fit was obtained with the wire. The bar, which was held by a lens mount, could be positioned on the optical bench such that the spoon would be on the optic axis of the instrument. The spoon was moved half way into the furnace, rotated $90^{\circ}$ and tapped so that the sample would fall of $f$. Standard errors of replicate $r$ uns indicated that sample transfer was reproducible to 108 .

Oil samples could not be transferred in a reproducible manner without first diluting with MIBK or xylene. The method of diluting both the oils and the solids has already been described in the matrix modification section.

Stock solutions containing 1000 ppm of the analyte were used for the preparation of the more dilute working standards. When an element was to be determined, working standards were used that were prepared on the same day. 
As

Arsenic was determined in orchard leaves, coal, coal fly ash, potery, oil shale and crude oil.

LLD: $-0.1 \mathrm{ng}$

Wavelength: $193.8 \mathrm{~nm}+197.3 \mathrm{~nm}$

Discharge: Magnetically Confined Lamp

Preparation of Standards: Add $0.1320 \mathrm{gm}$ arsenious oxide, $\mathrm{As}_{2} \mathrm{O}_{3}$, to a $100 \mathrm{ml}$ volumetric flask. Dissolve $\mathrm{As}_{2} \mathrm{O}_{3}$ in con $\mathrm{HCl}$ and dilute to $100 \mathrm{ml}$ with distilled water. This results in a $10 \% \mathrm{v} / \mathrm{v}$ $\mathrm{HCl}$ solution that contains 1000 ppm arsenic. Dilute this stock solution to the required concentrations with $18 \mathrm{HNO}_{3}$.

In order to prepare a standard arsenic solution in an organic solvent, add to a $100 \mathrm{ml}$ volumetric flask $50 \mathrm{ml}$ of ethanol and $5 \mathrm{ml}$ of concentrated $\mathrm{HCl}$. After the $\mathrm{As}_{2} \mathrm{O}_{3}$ dissolves add ethanol until final volume is $100 \mathrm{ml}$. This results in an $1 \mathrm{mg} / \mathrm{ml}$ solution. Dilute this stock solution to the required working solution concentration with methyl isobutyl ketone (MIBK).

Furnace: 1) Use four large (1/8" -3/16") holes in each end of a graphite heating element. This "gas window" increases sensitivity by about a factor of 5 . 2) Use center hole large enough to allow pipette to touch floor of graphite tube. 3) Use a pryolytic coating $^{29,30}$ since this lessens the penetration of the liquid into the graphite wall, and thus, solutions vaporize in a fashion similar to solids. Obtain pryolytic coating by heating furnace tube to $2200^{\circ} \mathrm{C}$ while flowing argon at $1.41 / \mathrm{min}$ and methane at $0.251 / \mathrm{min}$. Continue deposition for 10 minutes. 4) Mask the furnace to minimize the black 
body radiation from the pile of sample (diluted with graphite) on the floor of the tube. If radiation is not blocked off a low result will be obtained for the run.

Program: Three steps are involved, dry for 30 seconds, char for 30 seconds, and atomize until signal disappears.

1) Dry at 0.3 amps on auxiliary furnace power supply. This value was chosen by finding a setting that hastens evaporation but does not cause liquid to spray. At this setting the light intensity should fall to zero and return as vapors are given off. If drying is carried out at too high a temperature, the solution drop will spray or roll away from the center region resulting in a low signal. 2) Char at 0.6 amps on auxiliary power supply. This setting corresponds to 100 amps on the furnace power supply. Charing aids reproducibility even with liquid samples by insuring that there is no condensed liquid on the cool walls of the furnace that will come out during the atomizing step and react with the hot furnace. 3) Atomize at 3.0 amps (450 amps on furnace power supply). To make the heating cycle as reproducible as possible, first, turn off the furnace, then set the current on the auxiliary supply at 3.0 amps, then press the green start button. Turn off the furnace when the signal returns to zero.

Sample Preparation: The samples must be diluted in order to bring the amount of arsenic within the range of the linear portion of the analytical curve. This is accomplished, with solids, by mixing with spectrographically pure graphite using the Wig-L-Bug mixer. Usually it is necessary to dilute to a $18 \mathrm{mix}$. This should be done 
in two steps (10\% then 18$)$ in order to insure homogeneity. A sample spoon was used to transfer a weighed amount of $\mathrm{mix}$ into the furnace. For oils, MIBK was used to prepare dilute samples. It was found that the MIBK solutions were rapidly absorbed by the graphite heating elements, unlike the aqueous solutions that formed a non-wetting ball. In order to allow for the difference in solution penetration, arsenic standards were prepared in ethyl alcohol. The alcoholic stock solution was diluted with MIBK to prepare the working solutions. Since these solutions penetrated the heating element, it was possible to omit the drying step. The samples (and standards) were held at the charring temperature for 1 minute to lessen the smoke from the oil.

On some occasions there were differences in the signals obtained from the aqueous and the MIBK standards, and not on other occasions. It is believed that this effect depends on the condition of the graphite heating element.

Nickel addition: $5 \mu \mathrm{l}$ of $28 \mathrm{Ni}$ solution in $1.8 \mathrm{HNO}_{3}$ was added to the furnace before putting in either solid sample, liquid sample or liquid standard. This lessens the likelihood of sample loss during charring because of nickel arsenide formation.

The results of arsenic determinations in a variety of sample types is presented in the Table. The errors are twice the standard deviation. NBS values are results from the National Bureau of Standards. XRF values result fram $\mathrm{X}$-ray fluorescence determinations and NAA values result from neutron activation analysis determination. 
SAMPLE

$\mathrm{ZAA}$

OTHER METHOD RESULT (PPM)

\begin{tabular}{lcc}
\hline SRM 1571 Orchard Leaves & $10 \pm 2$ & $\begin{array}{l}14 \pm 2 \text { NBS } \\
\text { XRF }\end{array}$ \\
SRM 1632 Coal & $5.8 \pm 1$ & $5.9 \pm 0.6 \mathrm{NBS}$ \\
SRM 1633 Coal Fly Ash & $73 \pm 8$ & $61 \pm 6 \quad \mathrm{NBS}$ \\
Standard Pottery & $38 \pm 7$ & $30.8 \pm 2.2 \mathrm{NAA}$ \\
Raw Oil Shale & $83 \pm 16$ & $87.9 \pm 5.6 \mathrm{NAA}$ \\
\hline
\end{tabular}

$\underline{\text { Cr }}$

Lower Limit of Detection: $0.1 \mathrm{ng}$

Wavelength: $359.4 \mathrm{~nm}$

Discharge: Magnetically Confined Lamp

Preparation of Standards: Dissolve $0.3735 \mathrm{gm}$ potassium

chromate in $100 \mathrm{ml}$ volumetric flask with distilled water. Prepare working solutions as needed from this $1000 \mathrm{ppm}$ stock solution by diluting with $18 \mathrm{HNO}_{3}$.

The solid sample was diluted and transferred as already described in the matrix modification section and the arsenic section. The result is presented in the table.

\section{N.B.S = National Bureau of Standards}

\begin{tabular}{ccc}
\hline SAMPLE & ZAA & OTHER METHOD \\
RESULT (PPM) & RESULT (PPM) \\
\hline SRM 1632 Coal & $20 \pm 3$ & $20.2 \pm 0.5$ NBS \\
\hline
\end{tabular}


$\underline{\mathrm{Cu}}$

Lower Limit of Detection: $0.05 \mathrm{ng}$

Wavelength: $324.8 \mathrm{~nm}$

Discharge: Magnetically Confined Lamp

Preparation of Standards: Dissolve $0.1000 \mathrm{gm}$ of Cu metal in minimum volume of $1: 1 \mathrm{HNO}_{3}$ in a $100 \mathrm{ml}$ volumetric flask. Add distilled water to $100 \mathrm{ml}$ mark. Prepare working solutions as needed from this 1000 ppm stock solution by diluting with $18 \mathrm{HNO}_{3}$.

The solid samples were diluted and transferred as al ready described in the matrix modification sections and the arsenic section. The results are presented in the table. The error is 2 times the standard deviation. N.B.S. = National Bureau of Standards. $X R F=x-r a y$ fluorescence.

\begin{tabular}{lcc}
\hline SAMPLE & $\begin{array}{c}\text { ZAA } \\
\text { RESULT (PPM) }\end{array}$ & $\begin{array}{c}\text { OTHER METHOD } \\
\text { RESULT (PPM) }\end{array}$ \\
\hline SRM 1632 Coal & $15 \pm 5$ & $18 \pm 2 \mathrm{NBS}$ \\
Oil Shale & $40 \pm 7$ & $40 \pm 3 \mathrm{KRF}$ \\
\hline
\end{tabular}

$\underline{\mathrm{Hg}}$

Lower Limit of Detection: $0.1 \mathrm{ng}$ Wavelength: $253.7 \mathrm{~mm}$

Discharge: Electrodeless Discharge Lamp

Preparation of Standards: Dissolve $0.1080 \mathrm{~g}$ of $\mathrm{HgO}$ in a minimum volume of $1: 1 \mathrm{HCl}$ in a $100 \mathrm{ml}$ volumetric flask. Dilute with 
distilled water. Prepare working solutions as needed from this $1000 \mathrm{ppm}$ stock solution by diluting with $18 \mathrm{HNO}_{3}$.

Because of the difference in furnace configuration, the introduction of sample procedures are different for mercury than for any other element. In this case the solid sample is tared on a cup, the cup is placed into a long probe and the probe is inserted into an al ready heated furnace. A liquid sample is pipetted onto the cup already in place on the probe and inserted into the furnace. Extensive work as demonstrated that results are independent of the form of the mercury in the sample. ${ }^{31}$

Results are given in the table for National Bureau of Standards (NBS) samples. In addition one neutron activation analysis (NAA) result is given. The error is 2 times the standard deviation.

\begin{tabular}{lccc}
\hline SAMPE & $\begin{array}{c}\text { ZAA } \\
\text { RESULT (PPM) }\end{array}$ & $\begin{array}{c}\text { OTHER METHOD } \\
\text { RESULT (PPM) }\end{array}$ \\
\hline SRM 1571 Orchard Leaves & $1.5 \pm 0.6$ & $1.55 \pm 0.15$ NBS \\
SRM 1577 Bovine Liver & $0.016 \pm 0.006$ & $0.016 \pm 0.002$ NBS \\
Seal Kidney & $13 \pm 2$ & 10.5 & NAA \\
SRM 1632 Coal & 0.093 & $0.12 \pm 0.02$ NBS \\
SRM 1633 Coal Fly Ash & 0.14 & $0.14 \pm 0.01$ NBS \\
\hline
\end{tabular}


$\underline{\mathrm{Pb}}$

Lower Limit of Detection: $0.05 \mathrm{ng}$

Wavelength: $283.3 \mathrm{~nm}$

Discharge: Electrodeless Discharge Lamp or Magnetically Conf ined Lamp

Preparation of Standards: Weigh $0.1589 \mathrm{gm}$ of $\mathrm{Pb}\left(\mathrm{NO}_{3}\right)_{2}$ into a $100 \mathrm{ml}$ volumetric flask. Dissolve and dilute to $100 \mathrm{ml}$ with 18 of $\mathrm{HNO}_{3}$.

The solids were transferred and diluted as already described. A dual chamber furnace ${ }^{32}$ was used for the blood samples because of the tendency for the liquid to form into a large ball during charring and block off the light beam. The blood samples were round robin samples circulated by the California State Department of Public Health. N.B.S. = National Bureau of Standards. XRF $=\mathrm{X}-\mathrm{ray}$ fluorescence. The error is two times the standard deviation.

\begin{tabular}{|c|c|c|c|c|}
\hline SAMPLE & $\begin{array}{r}\text { ZA } \\
\text { RESULT }\end{array}$ & ( PPM) & $\begin{array}{l}\text { OTHER METHOD } \\
\text { RESULT (PPM) }\end{array}$ & \\
\hline SRM 1633 Coal Fly Ash & 65 & 13 & $70 \pm 4$ & NBS \\
\hline SRM 1577 Bovine Liver & 0.34 & \pm 0.08 & $0.345 \pm 0.08$ & NBS \\
\hline Oil Shale & $23 \pm$ & 6 & $23.6 \pm 1.9$ & $\mathrm{XRF}$ \\
\hline Blood CDC-5-21 & 0.35 & \pm 0.03 & $0.40 \mathrm{CSDPH}$ & \\
\hline Blood YB & 0.21 & \pm 0.04 & $0.26 \mathrm{CSDPH}$ & \\
\hline Blood G & 0.27 & \pm 0.02 & 0.33 CSPHD & \\
\hline Blood w & 0.04 & \pm 0.02 & 0.06 CSPHD & \\
\hline Blood B & 0.86 & \pm 0.2 & 0.92 CSPHD & \\
\hline
\end{tabular}


VIII. Conclusions

It seems fair to say that this project has bern eminentiy successful. In a very short period of time a new analytical technique was developed and was shown to be the solution to a major problem in environmental science (i.e., the determination of mercury in foodstuffs). This technique was next modified and extended to the determination of many elements in many sample types and was shown to have application to fossil energy research. Work now in progress has demonstrated that this technique also has application to the detection of molecules and has potential in speciation research.

Two technical breakthroughs were achieved (i.e., variable phase retardation plate, magnetically confined AC-RF light source) for which patents have been issued or are pending.

The technology has been transferred to the commercial sector to the degree that two major instrument manufacturers are investigating this technique in their laboratories and a third manufacturer has sold over 100 instruments based on this principle.

During the 6 years this project has been funded by the National Science Foundation the following accomplishments have been realized:

1) The feasibility of zeeman atomic absorption spectrometry was demonstrated for the determination of mercury in biological samples. A patent was obtained on this concept. ${ }^{3}$

2) A mercury determination method was developed that is fast and is not affected by the sample matrix.

3) The ZAA technique was extended to the same elements now determined by conventional atomic absorption spectrometry. 
4) Various optical designs and electronic processing systems were tried until an instrument of high sensitivity was constructed. A patent was obtained for this development. 4

5) A variable phase retardation plate was developed and patented. ${ }^{5}$

6) An intense light source that operates in the small space between the poles of a magnet was developed (patent pending).

7) A furnace was developed that will atomize most elements and that greatly reduces sample matrix effects.

8) The potential of forward coherent scattering as an atomic detection method was determined.

9) Methods of applying ZAA to the detection of molecules were developed (NO, HCHO, $\mathrm{I}_{2}$ ).

10) Molecular line shapes were determined using magnetic scanning techniques.

11) Molecular pressure broadening was measured.

12) Analytical methods that have application to a variety of sample types were developed.

13) The ZAA technique was modified to determine mercury in off gases from an oil shale retort.

14) A ZAA instrument was used in conjunction with a high pressure liquid chromatograph for speciation studies.

An important part of this project has been the transfer of this technology so that it will be used in the solving of environmental and energy research problems. This has involved contact with people from private industry who are in the process of producing a commercial 
instrument and work with people in environmental science on their particular analytical problems.

Three outside groups have been mainly involved: the Environmental Protection Agency Laboratory at Las Vegas, Nevada, the U. S. Corps of Engineers Waterways Experimental Station at Vicksberg, Mississippi and the N. S. I. Company at Mountain View, California. The Las Vegas laboratory is interested in using the Zeeman atomic absorption (ZAA) technique for the determination of mercury, lead and cadmium in biological samples; the Vicksberg laboratory is interested in the determination of mercury, selenium and arsenic in water with high salt content, and the N. S. I. Company is interested in producing a ZAA machine commercially. Work with these groups resulted in the sale of prototype instruments to both of these laboratories and the determination of selenium in some 250 samples at the Lawrence Berkeley Laboratory. In addition samples were analyzed for groups at the University of Alaska, the University of California at Irvine, and two different groups at the Lawrence Berkeley Laboratory.

Additional activities that resulted in hastening technology transfer resulted because of a consultant relationship that existed between the PI and Hitachi Instrument Co. of Japan. A commercial ZAA instrument has been produced and so far over 100 units have been sold. Neither NFS on LBL funding was used for these activities. During the past year representatives of the two largest producers of $\mathrm{AA}$ instruments in the United States have visited this laboratory. Both companies are carrying out research on the ZAA technique. 
There is no question that this work, funded by NFS-RANN, will. have considerable impact on analytical techniques that have application to environmental and energy related science. Without this funding the technique might never have been developed. The continued funding, for 6 years, was essential in order that the applications of this technique could be expanded and that technology transfer could occur. This work was supported in part by the U. S. Department of Energy. 


\section{Refer ences}

1) T. Hadeishi and R. D. McLaughlin, Science 174, 404 (1971).

2) T. Hadeishi, Appl. Phys. Lett. 21 (9), 438 (1972) .

3) T. Hadeishi, United States Patent 3, 811, 778 issued May 21, 1974 assigned to the U.S.A.E.C.

4) T. Hadeishi, United States Patent 3,914,054 issued Oct. 21, 1975 assigned to the U.S.E.R.D.A.

5) T. Hadeishi, United States Patent 3,957, 375 issued May 18, 1976 assigned to the U.S.E.R.D.A.

6) H. Koizumi - Lawrence Berkeley Laboratory, University of Calif. Berkeley, CA, private communication.

7) Hideaki Koizumi, Ralph D. McLaughlin and Tetsuo Hadeishi "High Gas Temperature Furnace for Species Determination of Organometallic Compounds with HPLC-ZAA System," LBL 8036 to be published in Anal. Chem.

8) P. Zeeman, Philos. Mag 43, 226 (1897).

9) W. Heisenberg and P. Jordan, Z. Physik 37, 263 (1926).

10) E. U. Condon and G. H. Shortley "The Theory of Atomic Spectra," Cambridge University Press, 1957, pg 378.

11) ibid, pg 379

12) ibid, pg 388

13) ibid, pg 383

14) ibid, pg 382

15) Hideaki Koizumi, Kazuo Yasuda and Mikio Katagama, Anal. Chem. $\underline{49}(8), 1106$ (1977).

16) B. V. L'Vov, Talanta 23, 109 (1976). 
17) J. W. Robinson, ed., "Handbook of Spectroscopy," Vol I, CRC Press, Cleveland, OH, 1974, pg. 804.

18) I.U.P.A.C. Information Bulletin No. 27, "Appendices on Tentative Namenclature, Symbols, Units and Standards," Nov. 1972.

19) L. Erdey, "Gravmetric Analysis," Vol 1, Pergamon Press, Oxford, 1963.

20) N. H. Furman, "Scott's Standard Methods of Analysis," Van Nostrand, New York, 1939.

21) Willard and Diehl, "Advanced Quantitative Analysis," Van Nostrand, New York, 1945.

22) Office of Standard Reference Materials Room B31l, Chemistry Building

National Bureau of Standards

Washington, DC 20234

23) G. F. Kirkbright and M. Sargent, "Atomic Absorption and Fluorescence Spectroscopy," Academic Press, San Francisco, 1974.

24) Conostan Division

Continental oil Company

P.O. Box 1267

Ponca City, OK 74601

25) Utra Carbon Corp., P.O. Box 747, Bay City, MI 48706.

26) F. J. Langmyhr, J. Find, J. Jonsen, Anal. Chim. Acta. 80(2), 297 (1975).

27) R. D. Ediger, At. Absorption Newlett 14 (5), 127 (1975).

28) B. V. L'vov, "Atomic Absorption Spectrochemical Analysis," Adam Hilger Ltd., 1970, pg. 231-237. 
29) S. A. Clyburn, J. Kantor and C. Veillon, Anal. Chem. 46, 2213 (1974).

30) D. C. Manning, R. D. Ediger, At. Absorption Newlett. 15(2), 42 (1976) .

31) D. A. Church, T. Hadeishi, L. Leong, R. D. McLaughl in and B. D. Zak, Anal.Chem. $46(9), 1352$ (1974).

32) T. Hadeishi and R. D. McLaughlin, Anal. Chem. 48(7), 1009 (1976). 
ZAA

INSTRUCTION

MANUAL 
I. Introduction ...................

II. Theory of Operation . . . . . . . . . . . . .

III. Discussion of Component Functions . . . . . . . .

A. Optics

1) Light Source... . . . . . . . . . . .

2) Phase Retardation Plate ...........

3) Furnace ..................

4) Photomultiplier Assembly . . . . . . . .

B. Electronics ..................

1) Automatic Gain Control ............

2) Lock-In Amplifier ..............

IV. Operating Instructions . . . . . . . . . . . .

A. Turn On Procedure ................

1) Light Source . . . . . . . . . . . .

2) Variable Phase - Retardation Plate . . . . .

3) Electronics

4) Furnace ..................

B. Operating Techniques ..............

1) Running a Sample... . . . . . . . . .

2) Smoke Problems ................

c. Typical Performance ...............

D. Shut Down Procedure ...............

v. Adjus tment . . . . . . . . . . . . . . . . .

1. Optical Alignment ................

2. Phase-Retardation Plate Tune Up............

3. Lock In Amplifier Tune Up............... 
VI. Maintenance ....................

A. Optics ....................

1) Light Source ................

2) Phase-Retardation Plate ...........

3) Furnace . . . . . . . . . . . . . .

4) Photomultiplier ..............

B. Electronics...................

1) Automatic Gain Control ............

2) Lock In Amplifier . . . . . . . . . . .

C. Hazards ................... 


\section{Introduction}

The Zeeman atomic absorption ( $Z A A$ ) technique represents a solution to what has been, for years, the most serious problem in atomic absorption (AA) spectroscopy; namely background correction. Because this method of correction is so superior to any method yet devised, it opens up the possibility of direct sample analysis for many sample types that until now have been impossible. This ability has been extensively exploited in the determination of mercury in a large variety of sample types that include fish, coal, oil shale, oil, orchard leaves and bovine liver. In the application of this technique to the determination of other elements a Massmann furnace of the type used in AA spectroscopy is utilized. Although the background correction capability is not altered, molecular formation and loss of analyte during ashing present problems not encountered with mercury. New furnaces have been developed to minimize these problems but it is still necessary to verify that a given procedure will work with a particular sample type.

II. Theory of Operation

The instrument proper consists of three major components. (1) the optical component with a high temperature furnace, (2) the electronic signal processor and (3) the furnace power supply. The component system is used to minimize the electronic interference and for easy future expansion of the system.

The operation of the instrument will be described in terms of the determination of mercury. The principles are the same for other elements, however the furnace is different. A block diagram of the optical component of the ZAA spectrometer is presented in Fig. 1. 


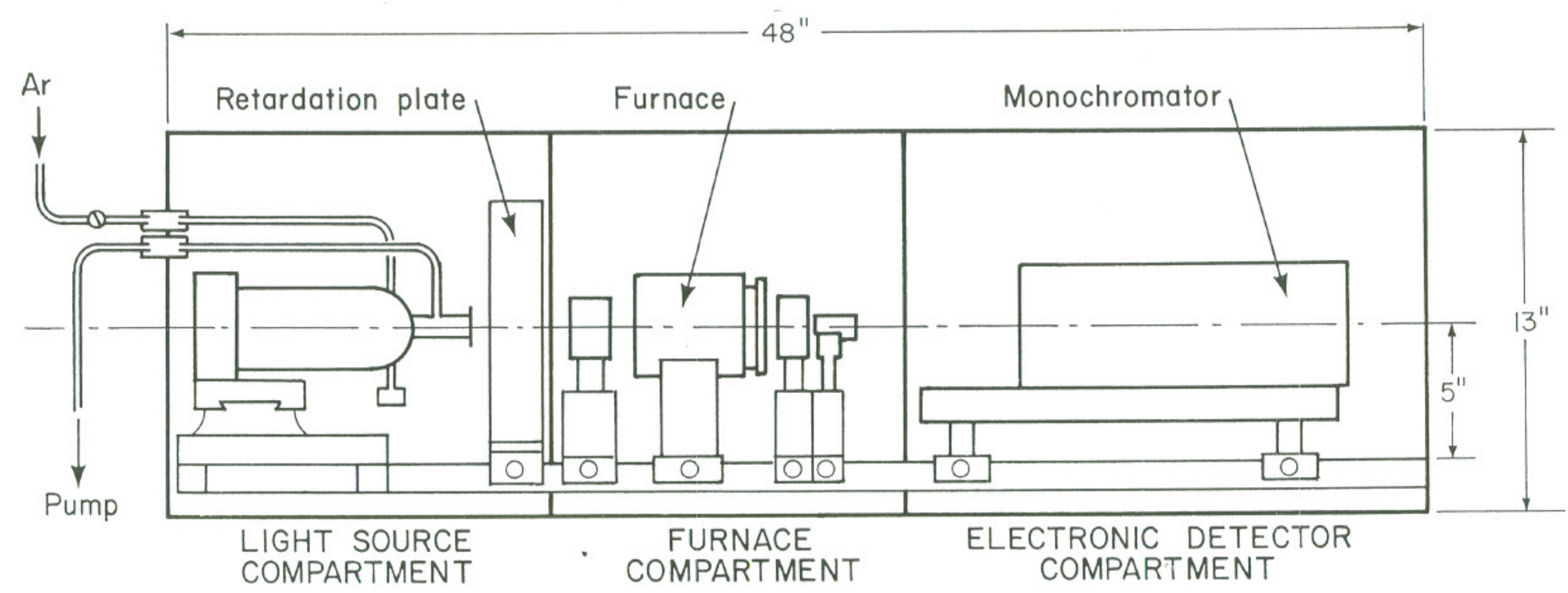

Fig. 1. Block diagram view of ZAA instrument. 
This figure depicts the magnetically confined lamp (MCL) and the Massmann furnace used for the determination of elements other than mercury. The MCL is replaced by an electrodeless discharge tube (see III-A-1.) and the Massmann furnace by a dual chamber furnace (see III-A-3) for mercury determinations.

The sample is thermally decomposed and combusted in a furnace maintained at a temperature of about $900^{\circ} \mathrm{C}$. The combustion and decomposition products are then swept into a heated absorption tube by a stream of oxygen. Here they are probed with a light beam consisting of two constituents; one has a wavelength centered on the absorption profile of natural mercury in air, while the other is slightly displaced (less than $1 \mathrm{~cm}^{-1}$ ) fram the mercury absorption line. The absorption of the centered constituent is due to mercury vapor as well as to non-mercury decomposition products - smoke, and any thermally stable molecular species present; the absorption of the displaced constituent is due only to the non-mercury background. In the vicinity of $2537 \AA$ line of mercury, this background absorption does not change significantly over $0.05 \AA$. Consequently, by taking the difference in the absorption of the two constituents, one measures the absorption due to mercury alone.

The heart of the technique lies in the mode in which the probe and the reference constituents are generated, and the method used to distinguish them from each other. In this instrument (see Fig. 2) both constituents are supplied by a single ${ }^{204} \mathrm{Hg}$ lamp operated in a 15 kilogauss magnetic field. When such a lamp is viewed perpendicular to the applied magnetic field, the Zeeman effect splits the $2537 \AA$ 


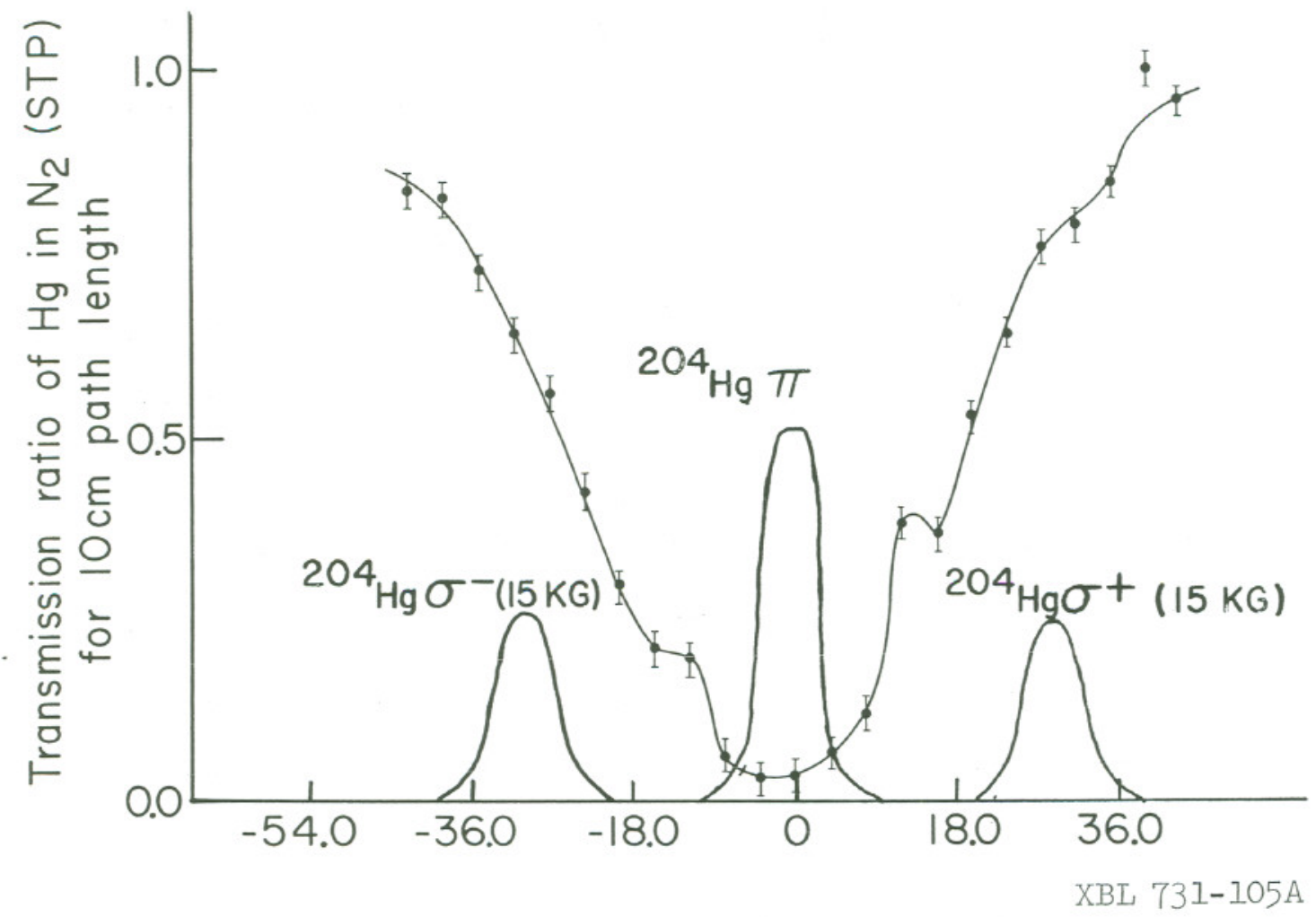

Fig. 2. The continuous curve is a plot of the absorption profile of naturally occurring mercury in $\mathrm{N}_{2}$ at S.T.P. The lower, superimposed curve gives the emission spectrum of the ${ }^{204} \mathrm{Hg}$ lamp operated in a 15 kilogauss magnetic field. The units on the abscissa are Ghz. At this wavelength $18 \mathrm{Ghz}=0.038$ and $36 \mathrm{Ghz}=0.076$. 
line into three components: a $\sigma^{-}$component shifted to longer wavelength, a $\sigma^{+}$component shifted to shorter wavelength, and an unshifted $\pi$ component. Now the mercury present in the absorption tube consists of a naturally occurring mixture of several stable isotopes. Since the absorption tube is operated at one atmosphere, the absorption 1 ines of each isotope are pressure broadened, and shifted slightly towards longer wavelength. In Fig. 2 we plot the resulting total absorption profile due to naturally occurring mercury in one atmosphere of $\mathrm{N}_{2}$; superimposed upon this profile is the Zeeman-split emission spectrum of the ${ }^{204} \mathrm{Hg}$ lamp. Note that the $\pi$ component corresponds accurately to the peak of the absorption profile of natural mercury, while the $\sigma$ components are both well off on the wings of the profile. Consequently we may use the differential absorption of the $\pi$ and $\sigma$ components as a measure of the quantity of mercury present in the absorption tube--the $\pi$ component becomes the probe beam, and the $\sigma$ components taken together become the reference beam. Although the details of the Zeeman patterns will differ, this background correction technique can be applied to any element.

Another feature of the Zeeman effect provides a convenient means of separating the $\pi$ and the $\sigma$ components. At right angles to the magnetic field, both $\sigma$ components are linearly polarized perpendicular to the field, whereas the $\pi$ component is polarized parallel to the field. Consequently, either component may be viewed independent of the other with a properly aligned linear polarizer.

In order to optimally utilize the ZAA technique, one must employ some method of alternately allowing the probe and the reference beams 
to fall upon a detector after being transmitted through the absorption region. The variable phase-retardation plate accomplishes this beam switching; it exploits the optical qualities of fused quartz. When fused quartz is stressed, it becomes birefringent--that is, light polarized along the stress axis propagates through the quartz at a different velocity than light polarized perpendicular to the stress axis. If a plate of fused quartz is oriented so that the stress axis makes an angle of $45^{\circ}$ with the plane of polarization of incoming light, the birefringence of the quartz introduces a phase shift proportional to the applied stress between the two perpendicular components of the light. By appropriately choosing the stress, the quartz can be made to function as a half-wave plate which causes the plane of polarization to be rotated by $90^{\circ}$.

In the present instrument, such a plate is oriented at $45^{\circ}$ with respect to the magnetic field applied to the light source. The quartz is mounted within a C-frame pulse-transformer core on which is wound a driver coil. Since the length of the quartz plate is chosen to leave an air gap of $0.5 \mathrm{~mm}$ on one side of the split core, varying the current in the driver coil varies the stress on the quartz plate; we have, in effect, a magnetic clamp.

After traversing the quartz plate of the variable phase-retardation plate described above, the light passes through the absorption tube, and falls upon a linear polarizer oriented parallel to the light-source magnetic field. When the current applied to the magnetic clamp is zero, the polarizer passes only the $\pi$, or mercury probe component of the light; when the current is adjusted so that the quartz is a 
half-wave plate, the quartz rotates the plane of polarization of both the $\pi$ and the $\sigma$ components by $90^{\circ}$, so now the polarizer passes only the $\sigma$, or reference components.

The light which passes the linear polarizer next encounters the monochromater which passes all components of the $2537 \AA$ line equally well, but which discards spurious light. After the monochromater the light finally reaches the photomultiplier, where it generates an electrical signal proportional to its intensity. If no mercury is present in the absorption tube, the probe and reference beams are absorbed and scattered identically by the non-mercury background. Hence, as they alternately fall upon the photomultiplier, the light intensity does not change, and the photomultiplier output signal remains constant. In the presence of mercury, however, the probe component will be more strongly absorbed than the reference component, and so the photomultiplier output will vary at the audio frequency at which the switching from one beam to the other takes place. This audio component of the phototube output is extracted and amplified by a lock-in amplifier.

In practice, in order that the lock-in amplifier output be proportional to the quantity of mercury present in the absorption tube, two additional devices are necessary. The first is an amplifier with electronically-controlled gain following the photomultiplier; its gain is automatically adjusted to compensate for the attenuation of the transmitted light by the non-mercury constituents, and for variations in the intensity of the light source. The second is a logarithmic 
amplifier which effects a more linear relationship between the number of atoms and the absorption signal than would a linear amplifier. III. Discussion of Component Functions

\section{A. Optics}

1) Light Source. The light source for elements other than mercury are depicted in Fig. 3. The resonant lines of the analyte are produced by a plasma that is excited by both $\mathrm{DC}$ and radiofrequency power. The plasma is maintained in a stainless steel tee that fits between the poles of a permanent magnetic field of about $13 \mathrm{KG}$. Since the magnetic field tends to confine the plasma to a plane perpendicular to the field, the light source is called the magnetically confined lamp (MCL). The discharge occurs in a flowing stream of argon. By use of a needle valve on the argon tank and a vacuum pump the pressure is maintained at between 1 and 10 Torr. An automotive spark plug functions as the anode and the cathode is a 10-32 screw with a cup drilled in the end to contain the element whose spectrum is to be excited. In order to obtain the spectrum of a different element, it is only necessary to change the cathode. In addition to the light source with its surrounding magnet, the radio frequency oscillator and the variable phase retardation plate are located in the light source compartment (Fig. 1). In order to determine mercury the MCL is replaced by an electrodeless discharge lamp (EDL) which is mounted in a block that facilitates air cooling. It is important that 


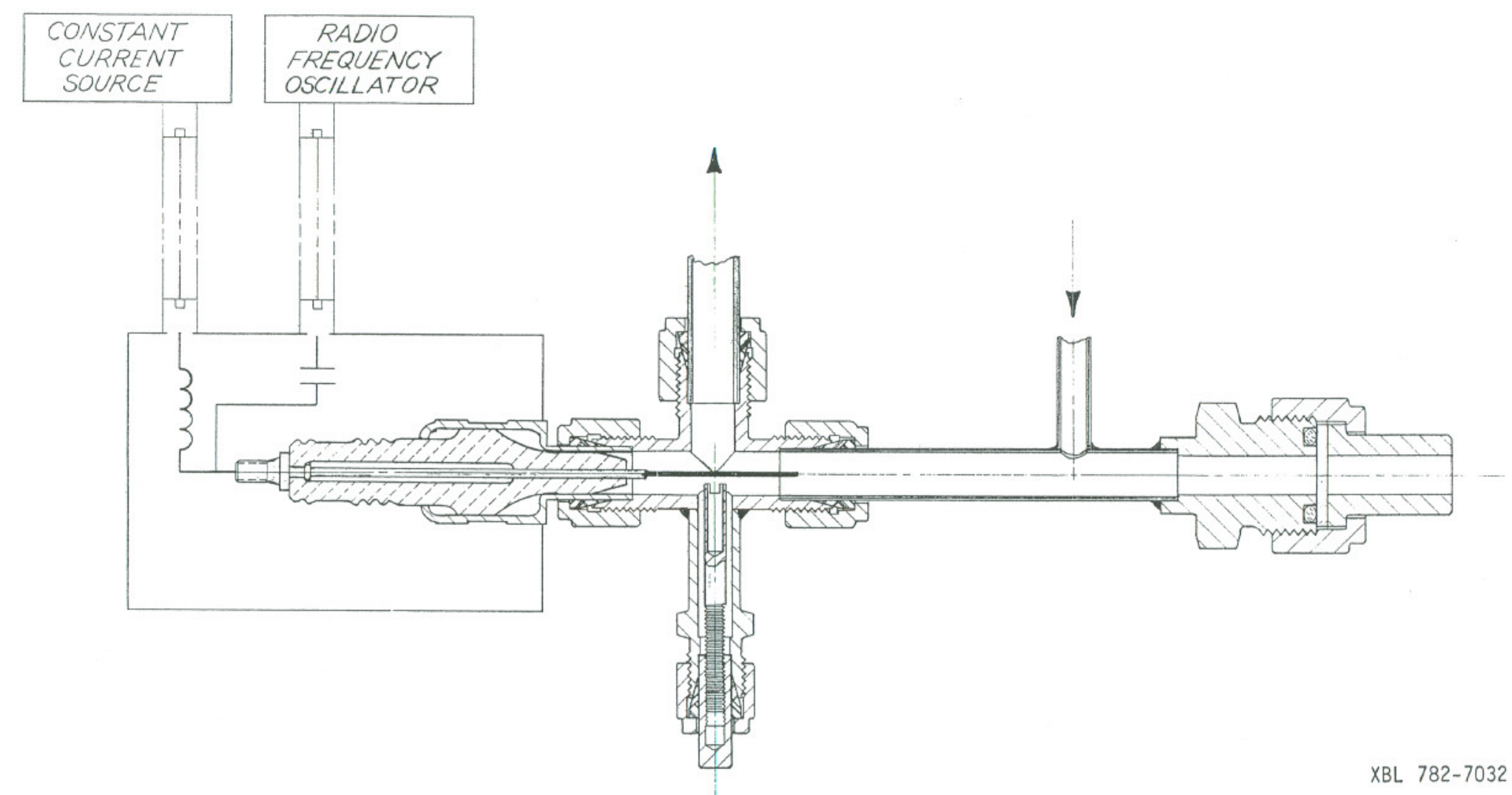

Fig. 3. Magnetically Confined Arc Discharge Lamp 
the EDL does not get too hot. If this occurs the mercury $253.7 \mathrm{~nm}$ line will become reversed. (This is caused by the absorption of the center of the emission line by mercury vapor in the discharge tube.) If the line is reversed the sensitivity deteriorates. The EDL cooling arrangement is pictured in Fig. 4. The brass block also restricts the region from which light is transmitted into the instrument to a small volume between the pole pieces. This is important since non Zeeman split mercury light that enters the system will decrease the sensitivity.

2) Phase-Retardation Plate. Figure 5 is a schematic of the retardation plate assembly. It contains a magnetic clamp which applies a sinusoidally varying stress to a plate of fused quartz. The quartz slab mounted in the magnetic clamp is approximately 1 in. long, $0.5 \mathrm{in.}$ wide, and $0.5 \mathrm{in.}$ thick. The light beam penetrates the quartz at the center of the slab. A mask blocks all light which does not pass through the quartz.

In order that the magnetic clamp function as described in section II, it is necessary to drive the clamp with an A.C. current superimposed upon a D.C. bias current. By adjusting the magnitude of the D.C. current, and the amplitude of the A.C. current, one can bring about the condition that at one extreme, the stress is just adequate to make the quartz function as a half-wave plate, while at the other extreme, the stress is near zero. It is important 

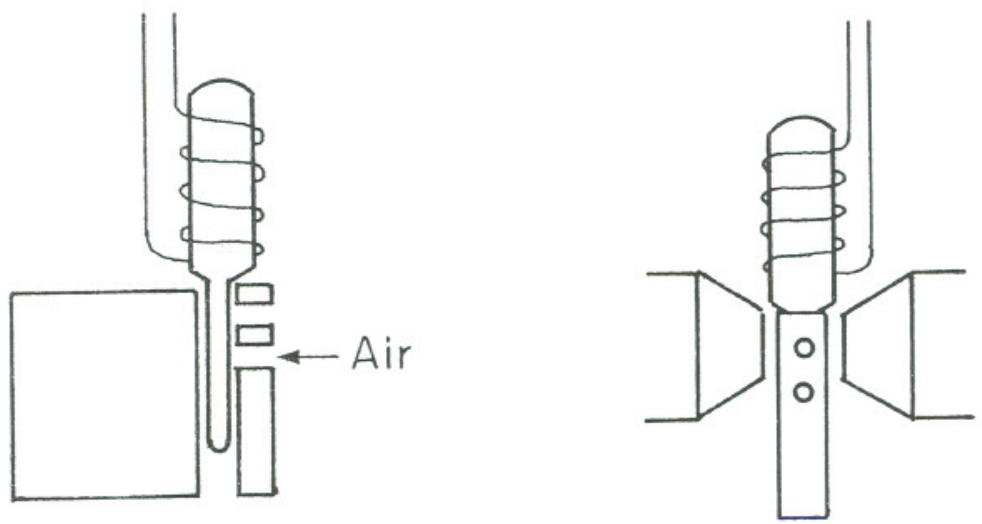

XBL 787-1397

Fig. 4. EDL Cooling Block 


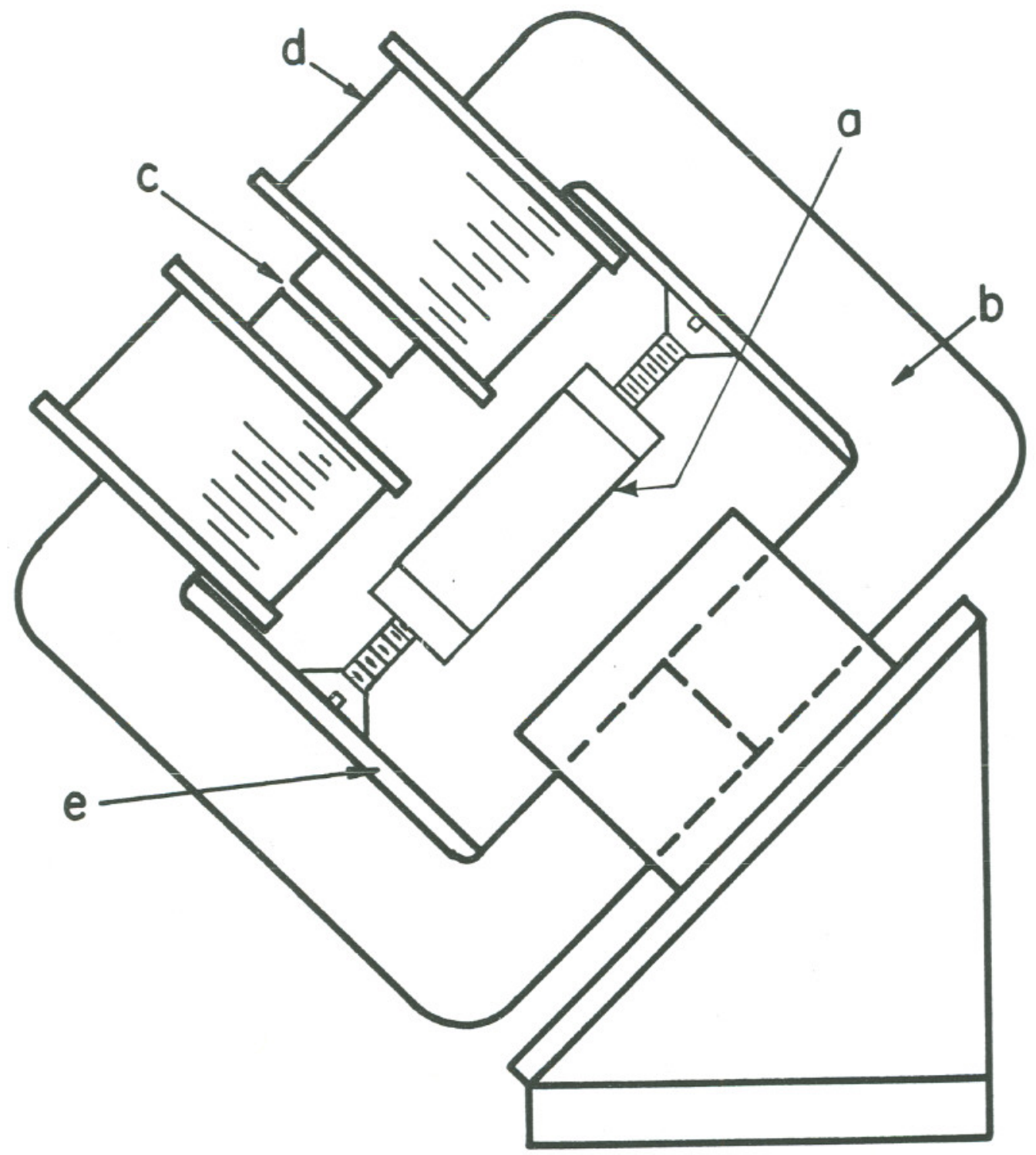

XBL 748-3969A

Fig. 5. Diagram of current-controlled phase retardation plate.

$a$, Plate of fused quartz; b, laminated pulse transformer core (Arnold Engineering AL 98 or Westinghouse L98);

c, 0.5-mm gap; d, drive coils (about 200 turns each;)

e, stiffener plates epoxied to the transformer core. 
that at no time the stress on the quartz actually pass through zero. If it does, the clamp loses contact with the ends of the quartz slab, causing chattering (clearly audible) and unstable operation. A block diagram of the electronics for driving the magnetic clamp is given in Fig. 6. Detailed instructions for adjusting the magnetic clamp will be given in section IV.

The adjusting controls for the varable phase retardation plate are contained in four NIM modules. One module supplies the DC clamp current. The second module is an audio frequency generator with variable frequency and amplitude controls. This unit determines the rate of modulation between the probe and reference wave-length in addition it supplies a reference frequency to the lock in amplifier. The third module contains a 100 Watt audio-power amplifier. The DC clamp current and the output of the audio power amplifier are mixed in the fourth module. The summed AC and DC current is then supplied to the variable-phase retardation plate. 3) Furnace. The function of the furnace is to convert the analyte in the sample to atoms and to minimize light scatter by volatilizing as much of the nonanalyte portion of the sample as possible during the drying and charring steps. For mercury determination this is accomplished in one step by inserting the sample into a high temperature region (Fig. 7) in an oxygen atmosphere. For biological 


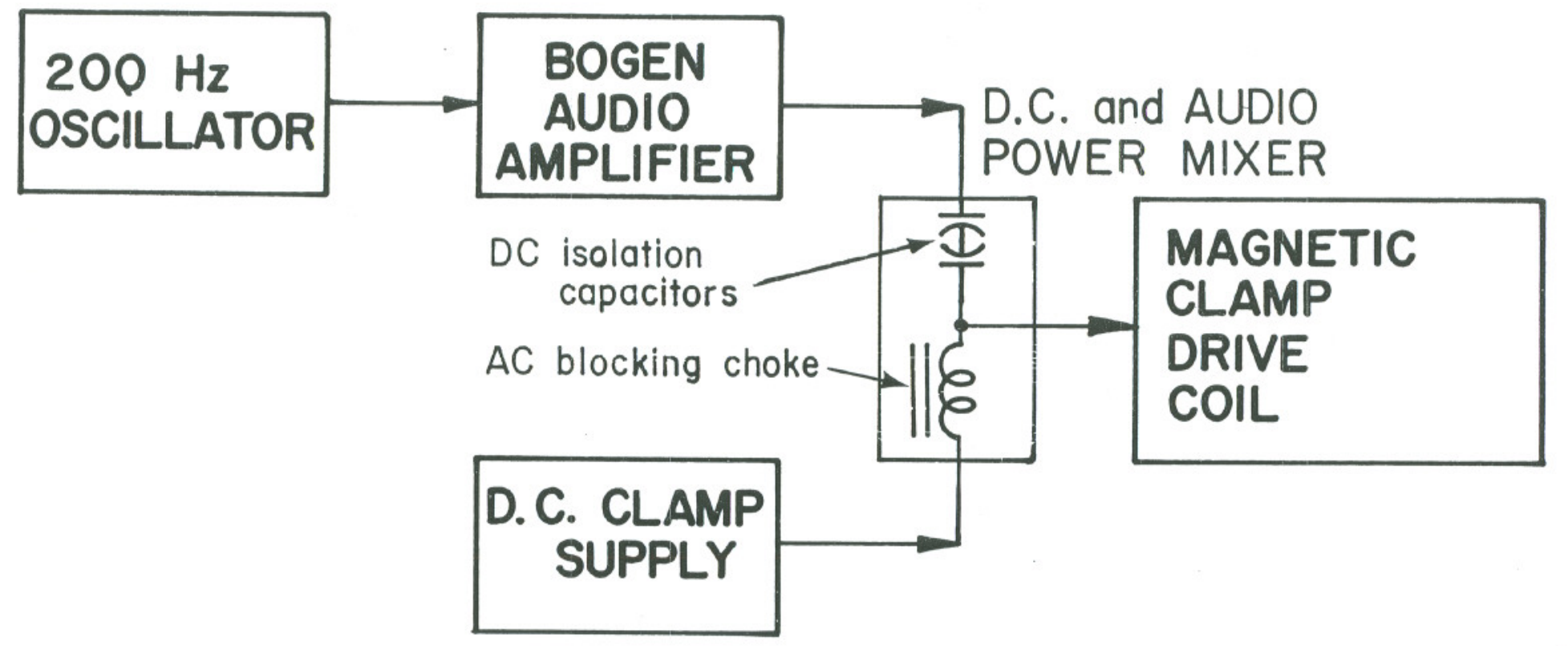

Fig. 6. Block Diagram of Magnetic Clamp Drive System. 


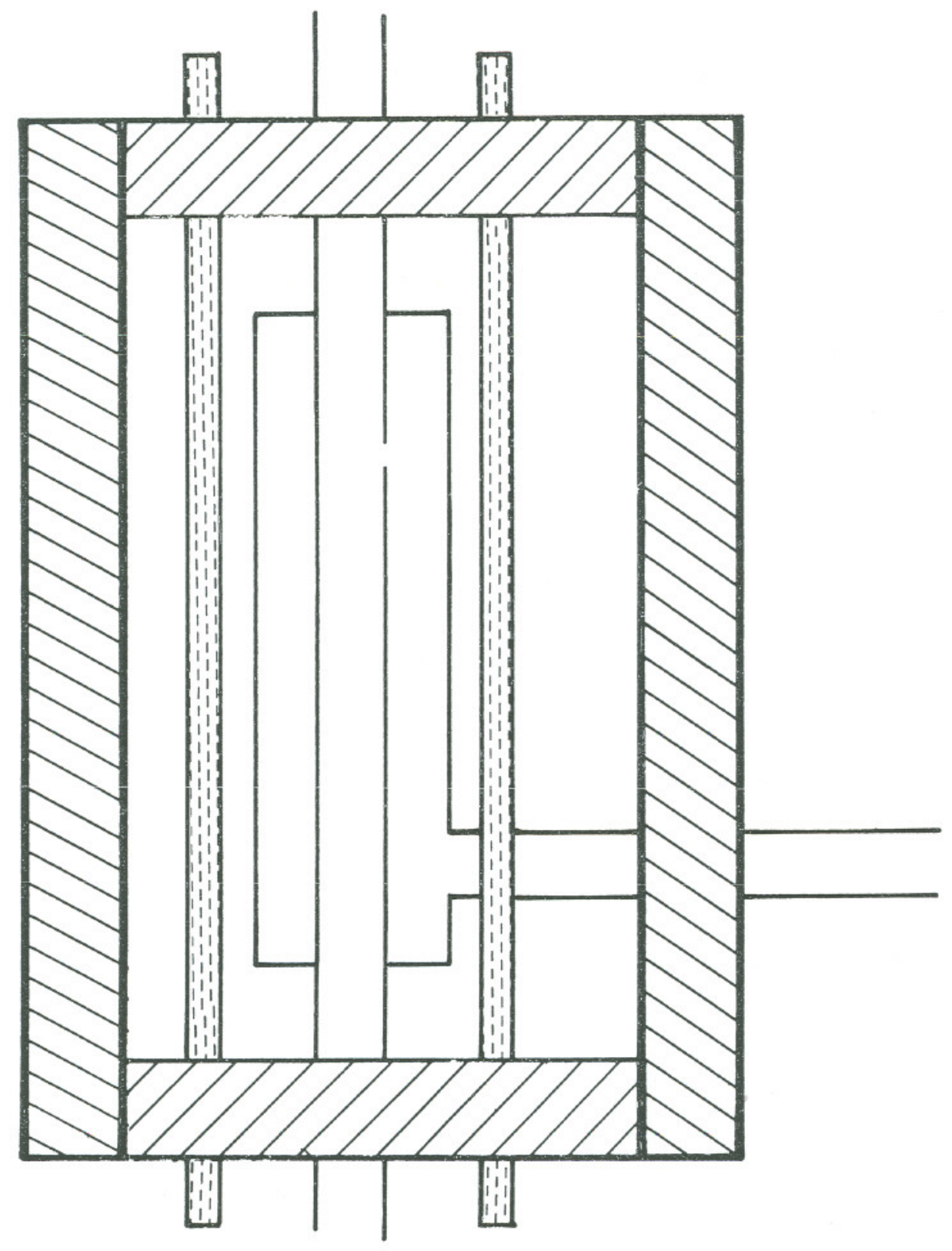

$X B L 755-2971$

Fig. 7. Furnace for Mercury Determinations. 
samples the organic material is converted to carbon dioxide and water and the mercuric oxide is decomposed into atomic mercury at the temperature of the furnace.

For all other elements it is necessary to raise the sample to higher temperatures to produce atoms. For this reason the conventional Massmann furnace is used (Fig. 8). It was found that PbS molecular formation could be avoided by using a dual chamber Massmann furnace also illustrated in this figure.

4) Photomultiplier Assembly. The photomultiplier assembly is mounted on the exit slit side of the monochromator in the right compartment of the main instrument. It consists of a DC-DC converter for the photomultiplier power supply, buffer amplifier for the photomultiplier signal output, and the photomultiplier itself.

The control unit for the DC-DC converter for the PMT power supply is one of the NIM modules in the NIM bin. The power supply control unit changes the DC-DC converter output voltage from 0 to 1000 volts. The photomultiplier should operate at about $500 \mathrm{~V}$ with buffer amplifier output of about $0.8 \mathrm{~V}$ for the best signal to noise ratio.

\section{B. Electronics}

The signal processing electronics consist of a photomultiplier tube, automatic gain control, logarithmic amplifier, lock-in amplifier and a read out device (i.e., two pin recorder or integrator) (Fig. 9). These components will be described in this section. 


\section{Oven assembly}
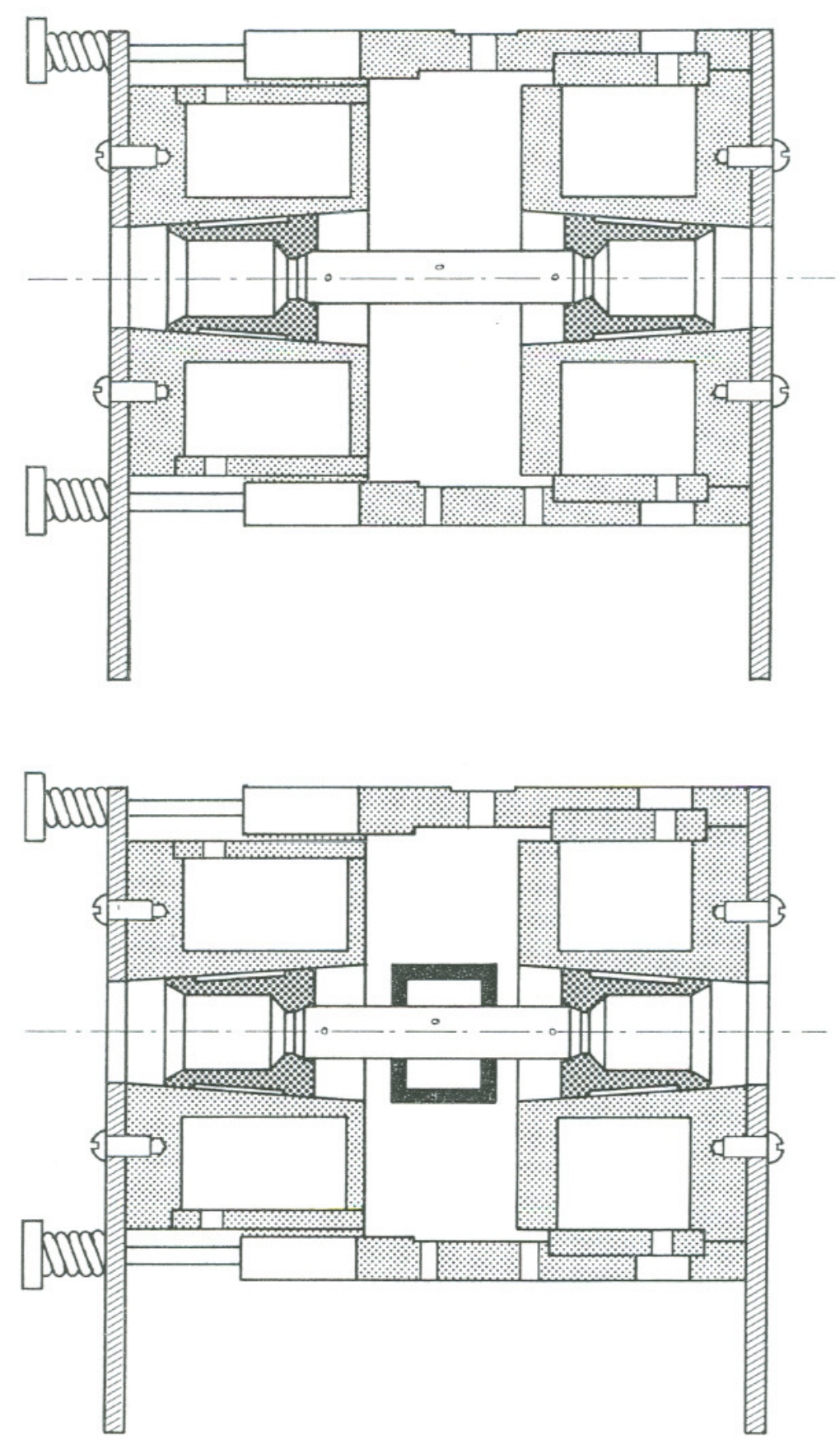

XBL 759-4117

Fig. 8. Above: Conventional Massmann Furnace. Below: Massmann Furnace modified for dual chamber operation. 


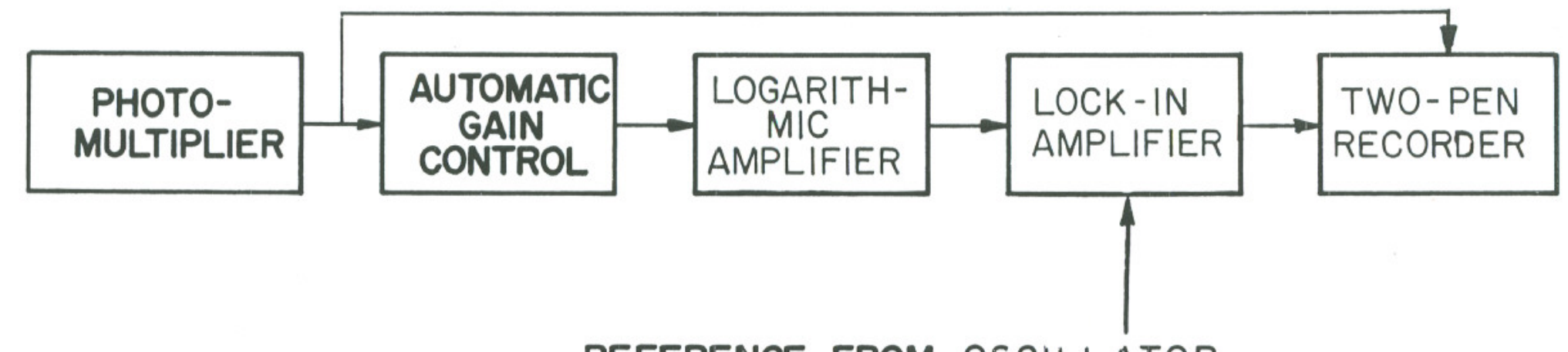

REFERENCE FROM OSCILLATOR

XBL $736-856 \mathrm{~A}$

Fig. 9. Signal Processing Electronics. 
1) Automtic Gain Control (AGC). The first element following the photomultiplier tube is the AGC. Its function is to correct for fluctuations in the light output of the lamp, and to correct for attenuation of the light beam by smoke. However it will not correct for emission from the furnace. The automatic gain control is followed by a logarithmic amplifier. The purpose of the $\log$ amplifier is to convert a linear signal into a logarithmic signal so that a signal proportional to the concentration of the atomic vapor in the absorption tube is obtained. When the light source intensity is very high, as is the case with the magnetically confined lamp, the AGC is not necessary. A toggle switch is provided to bypass the $A G C$ and use the log amp directly after the PMP, or, if desired, the AGC-log amp combination may be used.

2) Lock-in Amplifier. The purpose of the lock-in amplifier is to extract and amplify the audio frequency signal contained in the output of the photomultiplier due to the presence of the analyte in the absorption tube. In effect, the amplifier measures the signal level corresponding to the analyte plus background absorption, the signal level which corresponds to background absorption alone, and then generates a voltage proportional to the difference between the two. Since the "background", and the "analyte plus background" signals are presented to the lock-in amplifier alternately at the same terminal, the amplifier must also be provided with 
information as to when one signal is coming in, and when the other is. This information is provided by the reference signal from the audio oscillator. This signal, after amplification, is the signal used to drive the magnetic clamp. Consequently, it contains information as to when the clamp is applying stress and when it is not. This is equivalent to information as to when the background, and when the analyte plus background signals are being supplied to the lock-in ampl if ier.

The front panel of the lock-in amplifier contains many connectors and controls. To initially adjust these controls so that the amplifier is functioning properly requires one to go through a procedure outlined in section IV. Here we enumerate the controls and sketch their functions.

As soon as the signal enters the lock-in, it encounters a tuned audio frequency amplifier. The controls for this amplifier are grouped immediately above the input connector. This device selectively amplifies that portion of the input signal which corresponds to some chosen frequency; it suppresses components of the input signal which differ substantially from this frequency. The dial marked freq is the means used to set the frequency which will be amplified. The gain of the amplifier is set to one of the three values $1,0.1$, and 0.01 by the gain switch. For a variety of reasons, the stress on the quartz plate may not reach a peak at exactly the same time that the audio 
reference signal reaches its maximum value; that is, there may be a phase difference between the two. To correct for this, the phase of the audio reference signal may be shifted electronically relative to the input signal by adjusting the phase controls.

The circuit that takes the difference between the background, and the analyte plus background signals is called the mixer. It is this circuit that requires the audio reference signal in order to discriminate between the two. When the frequency and phase adjustments are properly made, this circuit provides a D.C. output voltage which is proportional to this difference; it is followed by a D.C. amplifier. Its gain is controlled by an appropriately labelled switch. When the difference between the two signals is small, the voltage output from the mixer and hence the amplifier may fluctuate quite a bit due to random instabilities in the system. What is significant is not the instantaneous, but rather the average voltage output of the amplifier. Means are provided to electronically average (damp) the output for a selection of averaging times. A toggle swi.tch allows one to change the averaging time from one second to 2 seconds. This time-averaged output is then presented to the meter at the upper left, and to the connector marked "D.C. out" (also duplicated on rear panel). It is this voltage which serves as the measure of the quantity of analyte in the absorption tube at any given time. 
The voltage presented to the meter and to the output connectors may be zeroed, or a constant voltage added or subtracted by use of the "zero offset" controls at the upper left. Finally, the sensitivity of the meter may be chosen to be either 1 volt or 10 volts full scale with the switch at the top right.

This completes the description of the lock-in controls with the exception of the tuned amplifier and mixer connectors. These connectors monitor the output of various points in the circuit as is required to check the operation of the lock-in and to "tune it up."

IV. Operating Instructions

The ZAA instrument for which this instruction manual is written is a research-prototype; as such, it makes greater demands upon operator knowledge and skill than would a commercial instrument. Consequently, a person is well advised to become thoroughly familiar with sections I, II, and III of this manual as well as section IV before attempting to operate the instrument. The location and function of the controls referred to here are described in the earlier sections.

It will be assumed in this section that the machine is in working order, and does not require extensive alignment or tune-up. Appropriate checks will be included, however, and, when required, the reader will be referred to a later section in which the necessary tune-up procedure is described. 


\section{A. Turn On Procedure}

Before proceeding, it is necessary to plug the instrument into a 115 VAC wall outlets.

At several points, the checkout procedure requires the use of an oscilloscope. The soope must be of the D.C. type, so it can display constant, as well as time varying voltages.

1) Light Source. The first step is to ignite the magnetically confined lamp. First, turn on the electronics bin; the power switch is at the lower right. Next, turn on the radiofrequency power supply (in the electronics bin). Wait two or three minutes. During this time, you may check the milliammeter on the radiofrequency power supply. It displays the emission current for the oscillator tubes that drive the lamp. As the tubes warm up, the current should rise to $60 \mathrm{ma}$ or so. Then turn on the constant current DC power supply with minimum current setting. The voltage should read maximum value and current value zero. Should voltage read zero, there is a short in the lamp; immediately turn off the power supply, disassemble lamp and correct shorting problem. Next turn on the argon carrier gas for the MCL. With gas on, the dc voltage should drop and dc current should read $20 \mathrm{~mA}$. Turn on the oscilloscope, put it in D.C. mode, and connect it to the P.M.T. Monitor. Adjust wavelength of monochromater to coincide with line of interest. One should observe a negative potential between 0.5 and 2 volts as dc current is slowly raised. This voltage should decrease 
to zero if the light path is blocked. If the mercury electrodeless discharge tube is to be used, it is only necessary to turn on the radiof requency power supply as described above. The discharge is started by use of a Tesla coil. 2) Variable Phase-Retardation Plate. Before starting this adjustment set the monochromator at the wavelength of the resonance line of the element in the lamp. Turn on the DC clamp power supply with the control knob at about the 0.5 position. Next turn on the audio-frequency signal generator and audio-frequency power amplifier; you should hear a gentle hum coming from the retardation plate module. If you hear a loud chatter, turn off the amplifier section and go to section $\mathrm{V}$. Next, place a linear polarizer supplied with the instrument in the front of the light source. The orientation of the polarization direction of the polarizer should be either parallel or perpendicular to the direction of the magnetic field of the lamp. You should observe a large sinusoidal wave in the PMI output on the scope. Adjust DC current supply and audio amplifier output for maximum amplitude at the fundamental frequency. It is possible to drive the retardation plate so hard that second harmonic occurs. This situation should be avoided. 3) Electronics. Set the gain of the lock-in amplifier at 0.01 . Remove the linear polarizer and adjust the zero off set to read zero on the meter. Now place the linear polarizer in the front of the lamp again as was done in 
instruction 2 above. Connect the scope to the connector marked tuned amp monitor. Adjust the control knob marked Freq. (frequency) to obtain a maximum amplitude. This adjusts the frequency response of the lock-in amplifier to be the same as the variable retardation plate modulation frequency for synchronous detection.

Next, connect the scope to the mixer output on the lockin amplifier. Set the lock-in amplifier gain to 0.01 . The pattern displayed on the scope (after its gain has been properly adjusted) should look like Fig. 10. If it does not, adjust the phase control until it does. Note that the meter reading is at a maximum when this condition obtains. Note down the meter reading with the polarizer in the beam for further reference. If you know what reading is displayed when everything is operating properly, putting the polarizer in the beam at a later time provides a quick check of proper instrument operation. If this test of the instrument indicates a loss of sensitivity, it is adviseable to go through the lock-in tune-up procedure given in IV-A.3. If that doesn't suffice to restore the lost sensitivity, one should also go through the retardation plate tune-up of IV-A.2. 4) Furnace. Turn on the 240 Volt power breaker. Set the control switch in the control box to internal. Set the toggle switch on the main power supply to $2000 \mathrm{sec}$ position. Set the current control knobs at T1 and T2 to minimum. Before pushing the furnace start button, turn on water cooling 


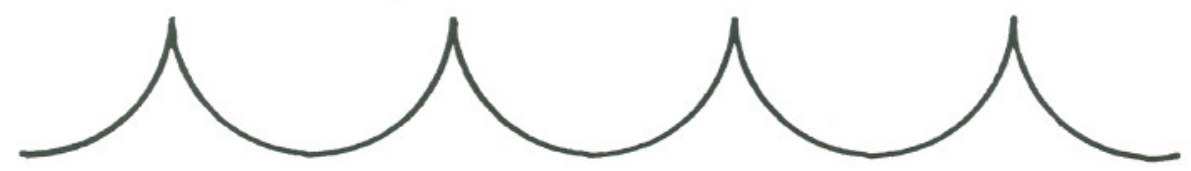

XBL $787-1398$

Fig. 10. Mixer output if phase is properly adjusted. 
and gas flow (described below). Next turn on the START button. You should hear a large click. This indicates that the solenoid switch is closed. Rotation of the knob (TI) should show a corresponding variation on the current meter 0-5 amp which is a measure of the reactor control current. At the same time, depending on the setting of the knob, a large current is now flowing through the graphite atomization tube. Use about 0.3 amp reactor current for drying for about 20-30 seconds and about 0.6 amp for ashing. The atomization current is dependent on the mobility of the element. For most elements, 2 amp should vaporize to the atomic vapor phase. For external automatic control, place the toggle switch on the control box to external control and go through a programming ( $d r y, a s h$, atomize) sequence. With the automatic control unit, the entire sequence of drying, ashing and atomization is done electronically. However, to determine the optimum program the manual control is recommended.

The furnace for mercury determinations is powered by 110 volts and controlled by a varac. Regulate the power such that the furnace reaches a temperature of $800-900^{\circ} \mathrm{C}$. Maintain this temperature as long as samples are being analyzed.

Oxygen is used in the furnace for mercury determination. The flow rate and adjustment procedure is the same as described for argon. 
5) Gas System. The gas system consists of an argon tank, a regulator, a solenoid valve, a flowmeter ( 1.6 liters/min full scale), and a flow-controlling needle valve at the flowmeter. Normally, it is only necessary to turn on the main tank valve, and to set the regulator at $10 \mathrm{psi}$ to bring the system into operation. Then, adjust the needle valve at the flow meter to set the flow rate to about $30 \%$ for most elements. For extreme sensitivity, which is seldom required, the gas interrupt technique may be used. For this purpose, the instrument is fitted with an electronically controlled solenoid with a SCR switch that operates with low dc voltage.

B. Operating Techniques

After one has followed the procedure outlined above, the machine should be ready to run samples. Here one should rezero the lock-in output using the off set control knob since drift usually occurs somewhat during warmup. Then, set the lock-in amplifier gain to the 0.1 position and connect one channel of the Two Pen Recorder to the DC output of the lock-in amplifier. The photomultiplier buffer amplifier marked PMT Output should be connected to the second channel of the Two Pen Recorder with 1 Volt full scale. Adjust either the PMT voltage or DC current for the light source such that the PMI output reads about $0.8 \mathrm{~V}$. Now, the electronic system is ready to operate. 
1) Running a Sample. Before starting a series of measurements, it is recommended that you clean out any remaining debris from the furnace and its vicinity. The instrument is very sensitive and contamination may cause a problem. Next, outgas the furnace by heating it to relatively high temperature and holding the temperature for a minute or two. Now you are ready to calibrate the instrument. This requires that you run a known quantity of sample with a known atomic concentration. Standard solutions dispensed with an Eppendorf pipette are most convenient for calibration. To avoid any errors from possible non-linearities in the system, it is desirable to use a calibration standard that contains a quantity of element comparable to that contained in the sample to be measured. Then one can $r$ un the standard and the sample with the same instrumental gain settings. It is convenient to begin with 5 microliters of a 1 ppm solution. Deposit 5 microliters into the graphite tube of the furnace using the Eppendorf pipette. Using the Dry, Ash and Atomization program, record the signals on the Two Pen recorder. If you wish to stay on the linear region of the analytical curve, the attenuation of the light by atomic absorption (PMT output) must not exceed 75\%. Repeat the standard calibration run a few times. Dividing the average result by the quantity of the element added gives one the calibration constant for this particular flow rate of argon gas, e.g., five microliters of a 1 ppm solution contains 
$5 \mathrm{ng}$ of analyte. If the average reading for these standards is 1 volt, then the calibration constant is $0.2 \mathrm{v} / \mathrm{ng}$. If all is well, one will get a distribution of results with a spread about the mean of \pm 108 or less. If the sample gives a signal of 0.5 volts then its analyte content is $0.5 / 0.2=2.5 \mathrm{ng}$. The sample should be $\mathrm{r}$ un several times to determine the precision of the measurement.

Solid and liquid samples are $r$ un in similar manner. Solid samples, or liquid samples that cannot be dispensed from an Eppendorf pipette, are somewhat less convenient to run, because one must know the weight of the sample introduced into the furnace. This may be accomplished by using a sample container and weighing before and after transferring the sample. For mercury determination the most convenient technique is to make an inner cup that fits inside the probe cup, but which is removable. The inner cup may be easily weighed before and after the sample is loaded into it; then it is placed in the furnace and $r$ un. 2) Smoke Problems. When organic samples are run which undergo incomplete combustion, they produce smoke. This smoke is troublesome in two ways. Although it attenuates both the probe and the reference beams equally, it would cause the instrument to read incorrectly if it were not for the action of the AGC. The AGC, however, can only accur ately correct for about $90 \%$ attenuation. Higher attenuation results 
in low readings. If the smoke level is too high, it is necessary to reduce the sample size.

C. Typical Performance

The remarks below pertain to the determination of mercury. Special considerations that pertain to other elements will be given elsewhere. In general, at the $1 \mathrm{ppm}$ level, one can expect that the measurements will be very straightforward. One can use relatively small samples--10 to $15 \mathrm{mg--and}$ still get large signals with no particular problems from smoke.

At the $100 \mathrm{ppb}$ level, one will want to begin to pay attention to smoke evolution. In most cases, limiting the smoke evolution so that the light attenuation is less than $30 \%$ is adequate.

At the $10 \mathrm{ppb}$ level, it may take considerable experimentation before appropriate combustion techniques are found for smoky samples. It is best if one can find atomization conditions under which there is no noticeable attenuation of the light as observed on the scope monitoring the light level. However, usually $10 \%$ attenuation is tolerable. At the $10 \mathrm{ppb}$ level, there is no guarantee that appropriate conditions can indeed be found for every possible sample type; yet experience indicates that the probability of success is high.

Much below $10 \mathrm{ppb}$, it is simply a matter of luck if one can find appropriate conditions for measuring the mercury concentration. The ultimte limitation is set by the output noise of the system. Typically, the noise permits the detection of $0.2 \mathrm{ng}$ with a signal to noise ratio of unity with a flow rate 
of 40; for smokeless samples, one can cut the flowrate to one quarter of this value or so, with a resulting increase in sensitivity such that the minimum detectable quantity becomes $.05 \mathrm{ng}$ of mercury.

of course, as the concentration falls, the uncertainty in the results increases. The degree of uncertainty depends, in part, upon the severity of the smoke problem. Also, since the noise becomes a significant fraction of the signal size at the lower concentrations, human error increases in the choice of the base line. It is important that the base line be correctly chosen in order to determine the peak height.

As an example of what can be done with low level samples, Fig. 11 shows the chart recorder output for two measurements made on N.B.S. Bovine liver samples which are certified to contain $1.5 \pm 1.5 \mathrm{ppb}$ mercury. Bovine 1 iver is an extremely smoky--even sooty--sample type. The noise shown here is typical.

\section{Shut Down Procedure}

Turn off all the power supplies, gases and water valves. It is advisable to post a note indicating WATER OFF, GAS OFF in front of the furnace to minimize the possibility of accidental operation of the furnace without sufficient water cooling or without the inert atmosphere necessary to protect the graphite heating element.

v. Adjustment
1) Optical Alignment. It is only necessary to re-align the optics after the instrument has been disassembled. 


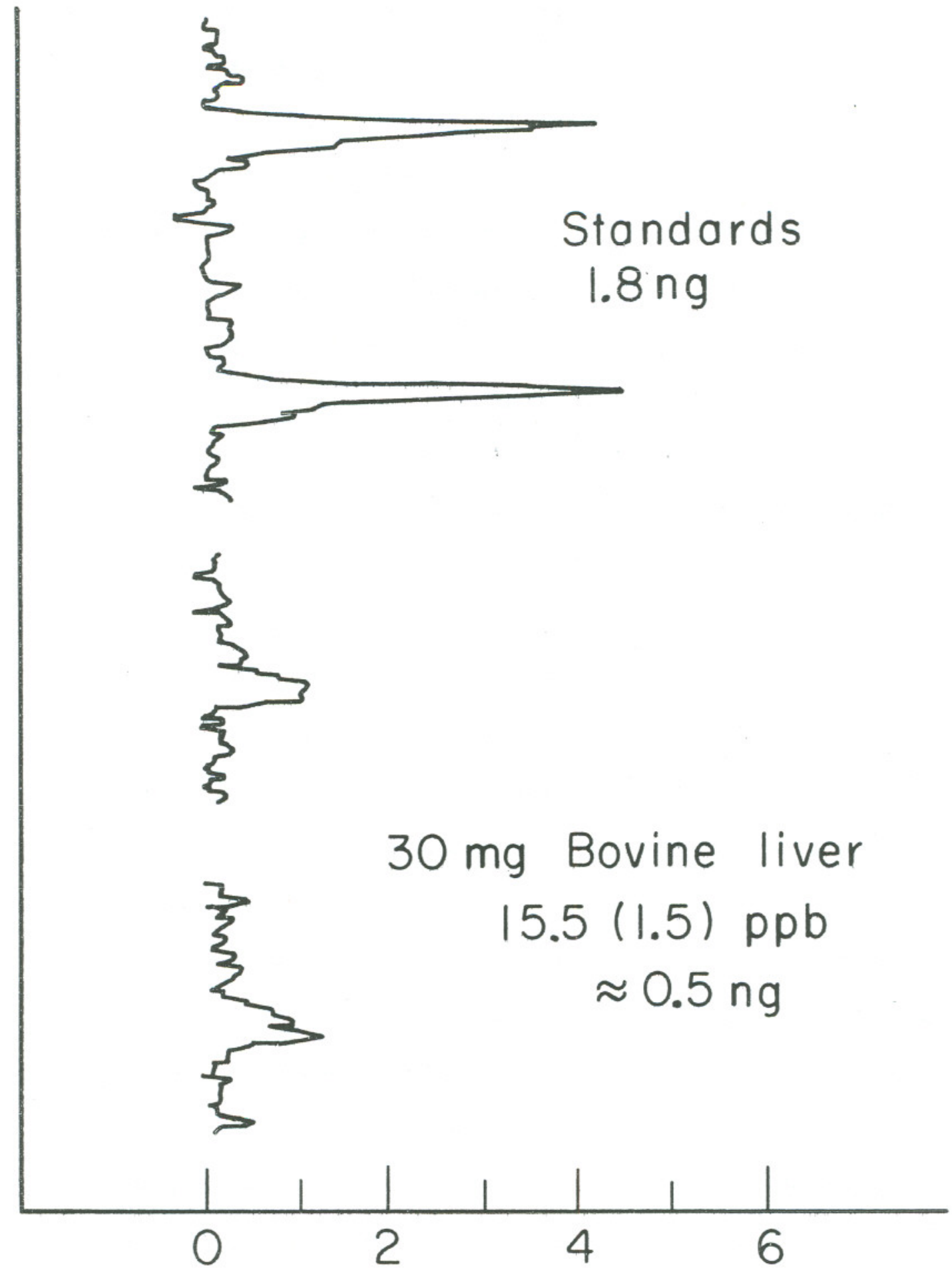

$X B L 737-3290$

Fig. 11. Two $15.5 \mathrm{ppb}$ bovine liver measurements plus two $1.8 \mathrm{ng} \mathrm{Hg}$ calibration runs. 
The optical alignment can be accomplished utilizing the small apertures furnished with the instrument. Define the center of the monochrometer by proper adjustment of a height gauge with respect to the center of the entrance slit. Replace the lens with the apertures. With the same height gauge, adjust the lens holders so that the apertures are on line with the center of the monochrometer. Now remove the apertures and place the lenses back into the holders. Now the optical system is perfectly aligned except the light source.

After placing the light source in the instrument, turn on and adjust height and later al position until a maximum light intensity is obtained on the scope or two pen recorder. Now the entire system is perfectly aligned.

2) Phase-Retardation Plate Tune-up. Insert a polarizer between the light source and the phase retardation plate. A sine wave will now appear on the scope if it is connected to the P.M.T. Monitor. Now increase the D.C. clamp setting and note that one side of the sine wave begins to flatten; decrease the clamp setting and note that the other side begins to flatten. Set the D.C. clamp to maximize the peak to valley voltage of the sine wave. This adjustment will maximize the output of the lock-in amplifier. Now increase the oscillator amplitude and note that the operation of the magnetic clamp gets louder, but also that the lock-in output increases somewhat. Note that eventually, the top 
and bottom of the sine wave flatten simultaneously, but that this happens when the operation is very noisy. Decrease the amplitude until the lock-in output has fallen $20 \%$ or so from its peak value. This amplitude should correspond to very quiet operation. The phase retardation plate is now properly adjusted.

3) Lock-in Tune-up. This procedure requires the lamp to be in operation. Connect the oscilloscope to the lock-in amplifier monitor. Next, place the linear polarizer furnished with the instrument immediately after the light source with the plane of polarization either parallel or perpendicular to the magnetic field.

A large sinusoidal wave should appear on the scope when it is connected to the P.M.T. Monitor. With the scope returned to the lock-in Monitor, rotate the signal channel Frequency control until the amplitude of the sine wave displayed on the scope is maximized. Finally adjust the phase control as described in section IV.A. 3 .

\section{Maintenance}

It is recommended that potential operators of the research prototype instrument do not wait until a malfunction occurs before reading this section; a general awareness of the types of malfunctions which can occur will improve the likelihood that trouble will be promptly recognized. It is the purpose of this section to prescribe remedies for the more common ills of the ZAA equipment. These occur for the most part in the optical, rather than in the electronic, section. The 
signal-processing electronics consists largely of solid-state integrated circuits; failure here is a rare occurrence. For this reason, detailed trouble-shooting of the electronics will not be considered. Nonetheless, all of the necessary diagrams for the equipment are included in section VI. With these diagrams, an electronic technician familiar with analog circuitry should be able to adequately service the equipment if the need arise.

\section{A. Optics}

1) Light Source. At its present stage of development it is best to replace the cathode of the MCL daily for the volatile elements (i.e. As, Cd and Se). The manner of producing these cathodes will be discussed under the headings of the individual elements. Maintenance is necessary even though material remains in the cathode. This is true because enough material is volatilized so that the discharge tends to jump to the walls where material has been deposited $r$ ather than being confined to the electrodes. The MCL should be disassembled and material cleaned from the walls and electrodes. Refill the cathode if necessary.

For successful MCL operation a clean system is essential. This means every effort should be made to keep organics out, to avoid leaks in the vacuum system and to minimize outgassing from the lines. If trouble is encountered in maintaining a stable discharge these are the most likely causes. 
The radio frequency power supply should be checked if it is not possible to initiate the discharge in either the MCL or the mercury lamp. First check the emission current shown on the lamp power supply meter. If the current is much less than $60 \mathrm{ma}$, either the power supply is mis-adjusted, or there is a fault in one or both of the oscillator tubes. Try increasing the lamp supply output voltage to bring the current up to 60-70 ma. If the lamp still does not light, shut off the lamp power supply, disconnect the power from the lamp module, and remove it from the bin; put the module on top of the bin and reconnect the power. Check to see that the filaments of both oscillator tubes light. If they do, rotate the voltage adjust on the lamp power supply full clockwise. The milliammeter should read over $100 \mathrm{ma}$. If a tube is not lit, or if the current will not exceed 100 ma, replace both tubes.

It should be noted that, after a few hundred hours of operation, the mercury lamp frequently begins to get "noisy;" that is, the random variations in the output of the lock-in amplifier grow larger. Particularly if one is making measurements at the limit of sensitivity of the instrument, this degradation in performance is troublescme. Therefore, it is best to replace the lamp when the increased noise becomes detrimental, rather than waiting for the lamp to fail totally. 
2) Phase-Retardation Plate. There are two faults which can occur involving the retardation plate. The device may chatter or the device may not operate at all.

The magnetic clamp chatters when the stress applied to the quartz plate goes to zero at some point in each stress cycle. The first thing to suspect is that either the D.C. clamp supply or the A.C. oscillator amplitude or both are mis-set. Check appendix IV.A2. If it is not possible to set the controls so that the chatter is eliminated, either the D.C. supply is non-functional, or there is a loose connection in the wiring. To check this, turn the audio amplifier off, turn the D.C. supply down to zero, and insert a D.C. ammeter in the drive circuit (at the Jones strip on the rear panel of the module). The D.C. supply should furnish at least 5 amps when it is turned up to full output. If the D.C. supply is functioning properly, but the chatter remains, disconnect the various leads from the back of the module, and remove the module from the bin. Examine the magnetic clamp itself to see if any parts have become loose. If there is no evidence of function of the retardation plate at all, refer to appendix IV.A2. If the procedure described there cannot be carried out, or does not solve the problem, it is probable that either the audio amplifier is not functioning, or a loose connection in the inter-module wiring is preventing the audio frequency power from reaching the magnetic clamp. Use the oscilloscope to trace the signal 
from the oscillator through to the magnetic clamp. If the problem resides in the audio amplifier consult the product literature for this device.

3) Furnace. The Massmann furnace will slowly change its apparent atomization efficiency because the graphite tubes become porous. A porous tube results in fewer atoms in the light path because of diffusion through the walls. In order to determine the efficiency notice how the signals from a standard solution change as a function of furnace use. When the efficiency becomes unacceptable, replace the furnace element.

If large amounts of an element are volatilized it is possible to contaminate the furnace with this element. This results because material has condensed on the walls which then falls on the heating tube during the insertion of the sample. This will cause erratic results to be obtained if a standard is run repeatedly. If this happens the furnace must be disassembled and scrubbed with an abrasive cleanser. The problems that may arise involving the mercury furnace all have a common cause; the furnace itself, its external heaters, and the sample probe inserted into the furnace all slowly oxidize. The higher the temperature, the faster the oxidation occurs. Consequently, low operating temperature favors long life for these components. Unfortunately, below some particular temperature, not all of the mercury compounds in the sample will be decomposed to atomic mercury vapor 
in the furnace. A suitable compromise seems to be operation in the $\mathrm{r}$ ange $800-900^{\circ} \mathrm{C}$.

Under normal conditions, the probe may have a practical life of a few hundred determinations; utimately, the probe begins to disintegrate, and so must be replaced. Similarly, the furnace itself or its external heaters may fail. However, if the furnace does not come up to operating temperature it is wise to check the fuse in the furnace Variac before proceeding further. If the problem does not lie with the fuse, disconnect the external heaters and the main furnace leads, and check the furnace and the heaters with an ohmmeter. If there is evidence that either the furnace shell or the external heaters are burned out, remove the furnace assembly from the furnace bin.

Remove the furnace and unwrap the insulation. The external heaters and the furnace itself should be examined to identify the point of failure. If it is the furnace shell itself which failed, the furnace will have to be replaced; if it is an external heater, all heater units should be replaced, and the furnace re-insulated. Then the furnace should be re-installed, its position determined by the alignment procedure described in appendix IV.Al. It is anticipated that in the near future more easily serviced versions of the furnace will become available for replacement purposes. 4) Photomultiplier. The only likely maintenance chore connected with the photomultiplier is the adjustment of 
the voltage applied to it. One would like to keep the D.C. level at the P.M.T. Monitor at about 0.8 volts when the lamp is thoroughly warm, and when the reference and the probe beams are properly balanced. If the D.C. level is above four volts, the P.M.T. will begin to saturate, and the instrument will give erroneous results. The adjustment on the P.M.T. panel should be used to establish this condition. The adjustment will have to be made periodically as the lamp ages, and also when a new lamp is installed.

\section{B. Electronics}

1) Automatic Gain Control. If there is doubt whether the $A G C$ is functioning properly, it is suggested that one check it using a dual channel oscilloscope. Connect one channel to the P.M.T. Monitor, and the other to the front panel lock-in amplifier input; this effectively monitors the AGC output. Next, slowly move an obstacle through the light beam. The P.M.T. Monitor will reflect the decrease in light intensity as more and more of the beam is intercepted. If the AGC is operating properly, however, the output of the AGC will show no change in D.C. level until approximately $90 \%$ of the beam is blocked; then the output voltage will rapidly approach zero.

Proper operation of the AGC depends upon proper adjustment of the "Sig. Level Adj.;" in normal operation, with no sample being run, this control should be set so that one observes about 1.5 volts at the "Feedback Monitor" connector. If 
the light level declines, this voltage rises; if the light level rises, this voltage declines. The suggested setting allows adequate dynamic range.

2) Lock-in Amplifier. If it is impossible to set the phase control so that one obtains the pattern shown in section IV.A.3, it is probable that the "Sq. Ref. Symmetry" control requires adjustment. If such is the case, follow the instructions given in that section, but adjust the phase control and the symmetry control simult taneously until one has obtained the required pattern. Only when parts have been replaced in the reference channel of the lock-in amplifier is it likely that this adjustment will have to be made.

\section{Hazards}

There are two hazards associated with servicing the ZAA instrument: the first is an eye hazard from the ultraviolet light generated by the light source; the second is an electric shock hazard from the high voltage (up to $300 \mathrm{~V}$ ) applied to the light source rf oscillator. Because the ultraviolet light does not pass through most glasses and plastics, a person wearing eyeglasses is most likely well protected. However, if it is necessary to look at the light source for a sustained period of time--for instance, while aligning the optics--a person not wearing glasses should look through a piece of window glass, or or dinary (non-UV transmitting) lucite. The shock hazard from the light source comes about when one is servicing the lamp module with the module removed fram the NIM bin, but with the 
power on. Note that the lamp drive electrodes may be at high voltage. Use an insulating tool to adjust them. 


\section{AUDIO-FREQUENCY \\ SIGNAL GENERATOR MODULE}

$1058 \times 103 M$

4.

W RESISTOR. 


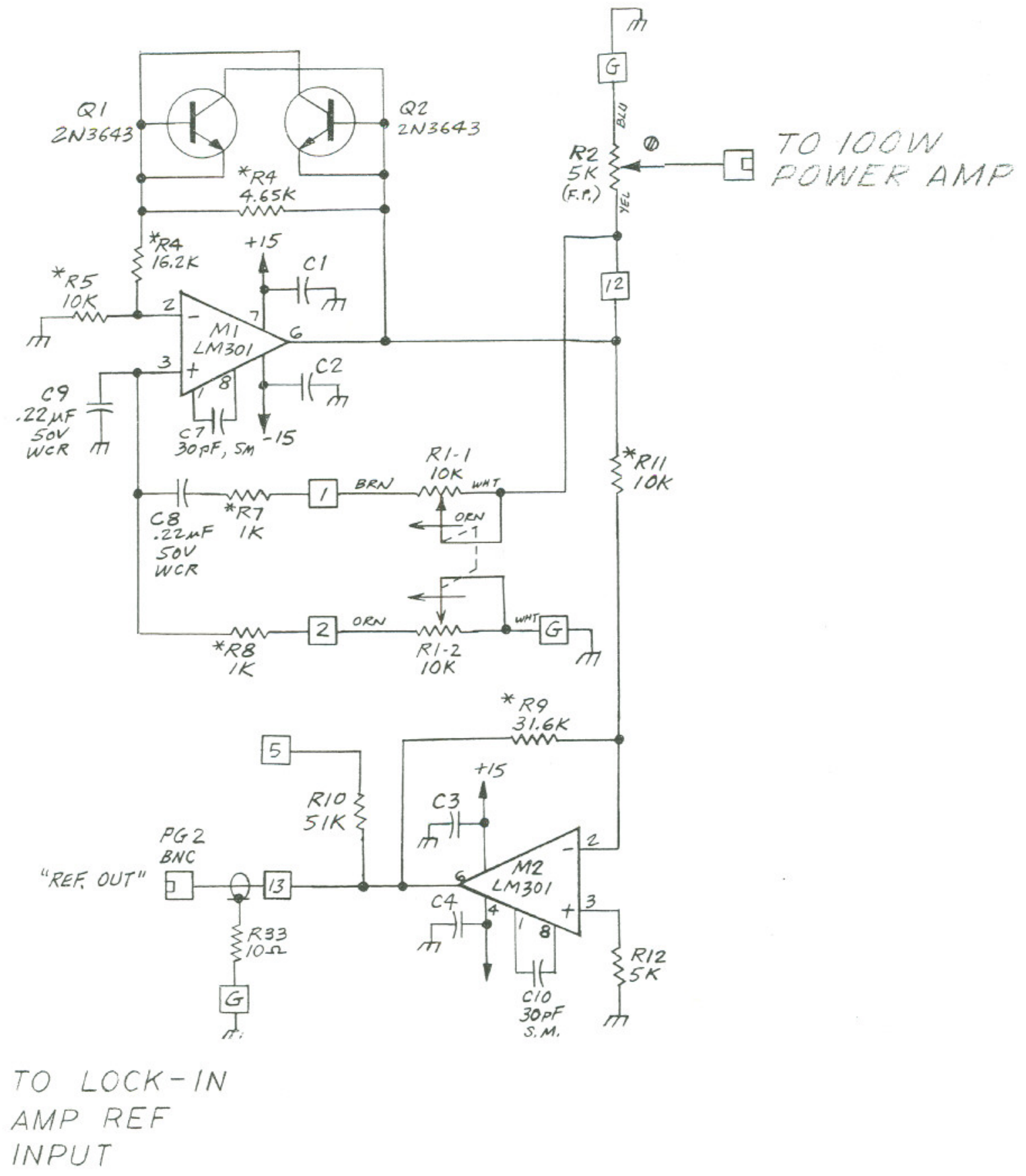

NOTES - INLESS OTHERWISE SPECIFIED

1. ALL RESISTORS 1/4W 5\% CARBON

* = $1 / 8 \mathrm{~W} / \%$ METAL F/LM T-O

2. ALL CAPACITORS . OIMF IOOV CERAMIC CKO5BX103M

3. USE 8-PIN SOCKETS FOR MI, 2 \& 3

4. USE TRANSISTOR SOCKETS FOR Q1, 2,3 \& 4 .

** 5. LI = 30 TURNS 2OAWG FORMVAR AROUND zOK $2 \mathrm{~W}$ RESISTOR. 


\section{AUDIO POWER AMPLIFIER D.C. CLAMP MIXER}

D.C. CLAMP INPUT

AUDIO AMP INPUT

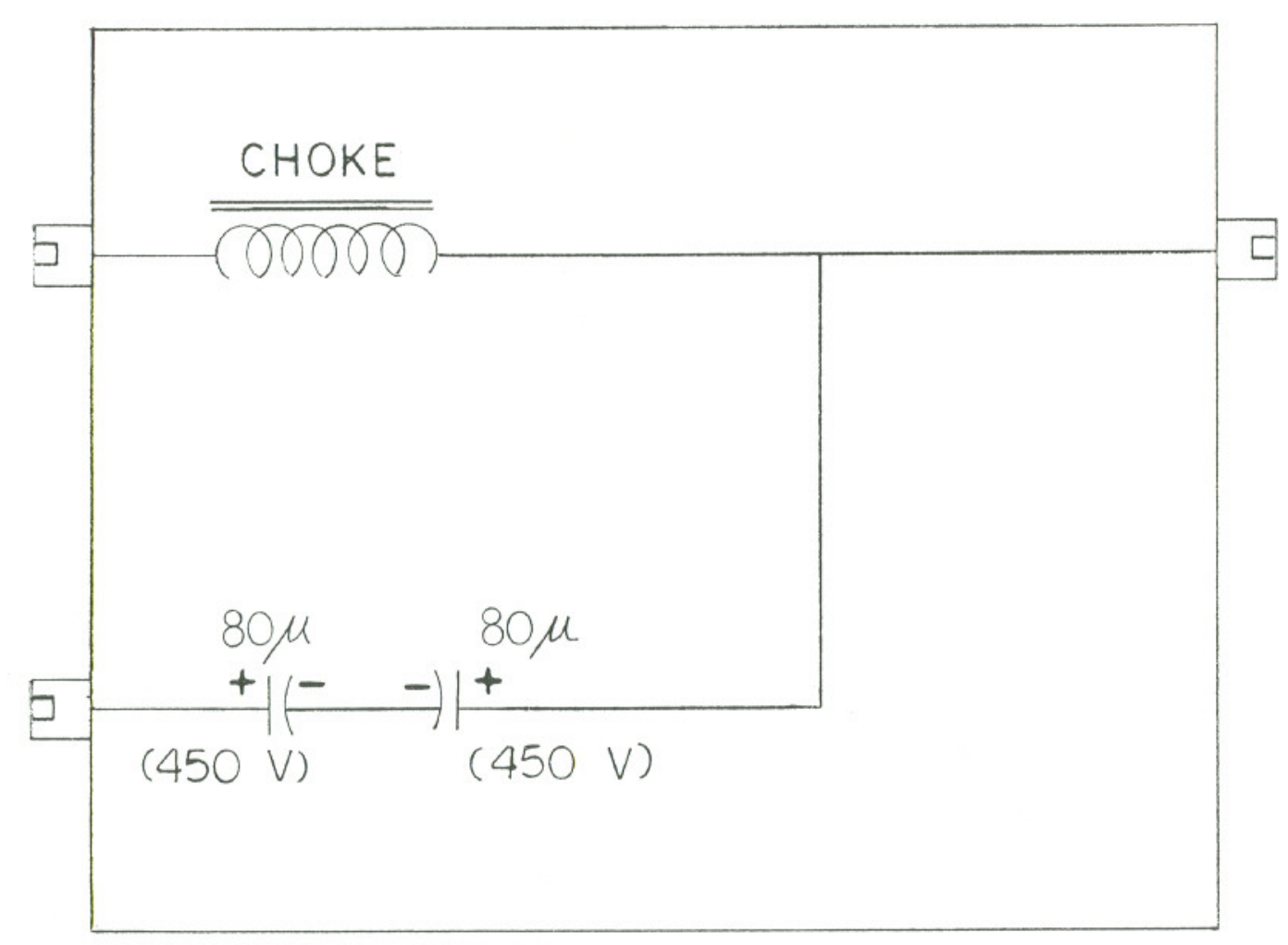

TO SQUEEZER 
POWER AMPLIFIER

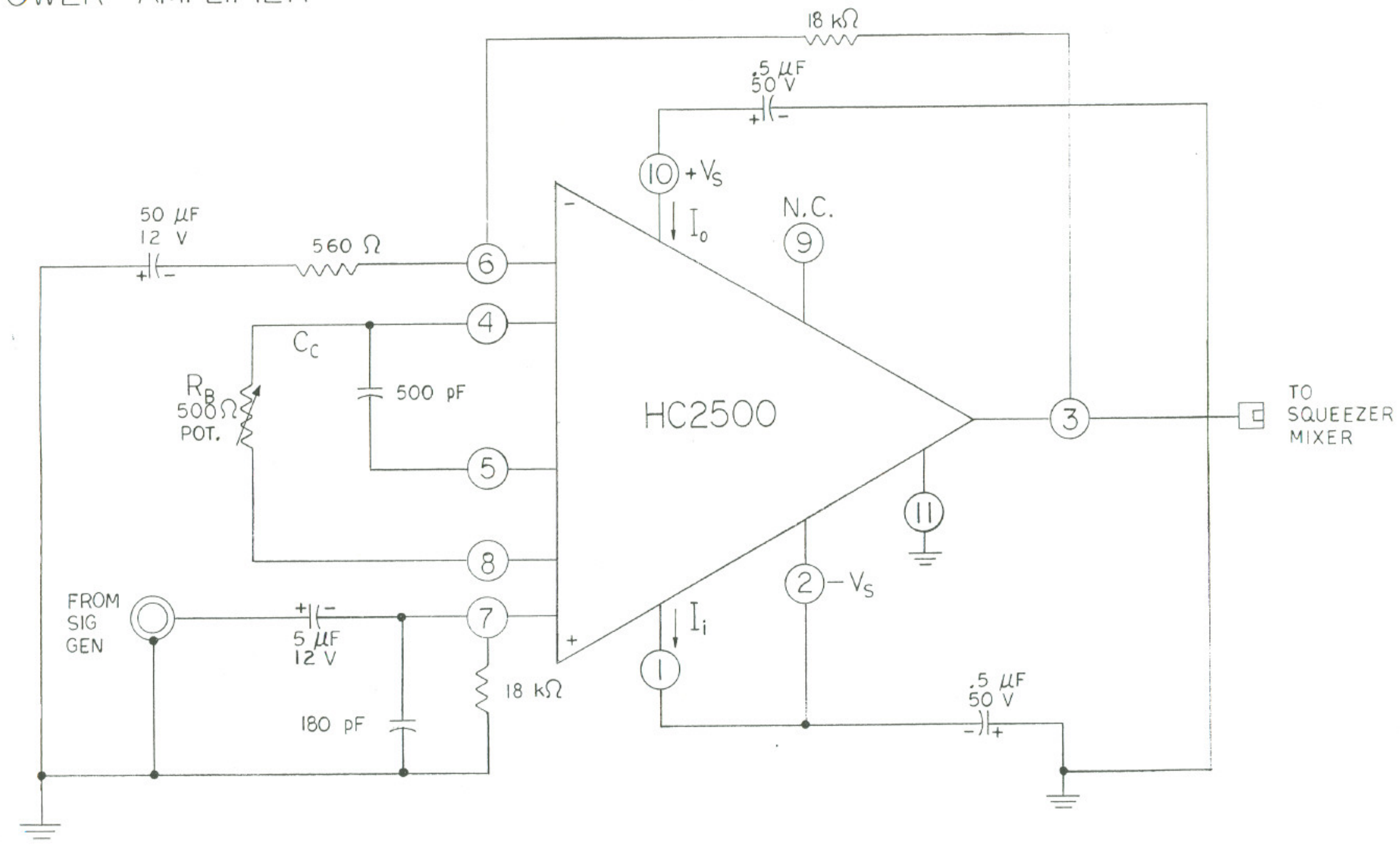




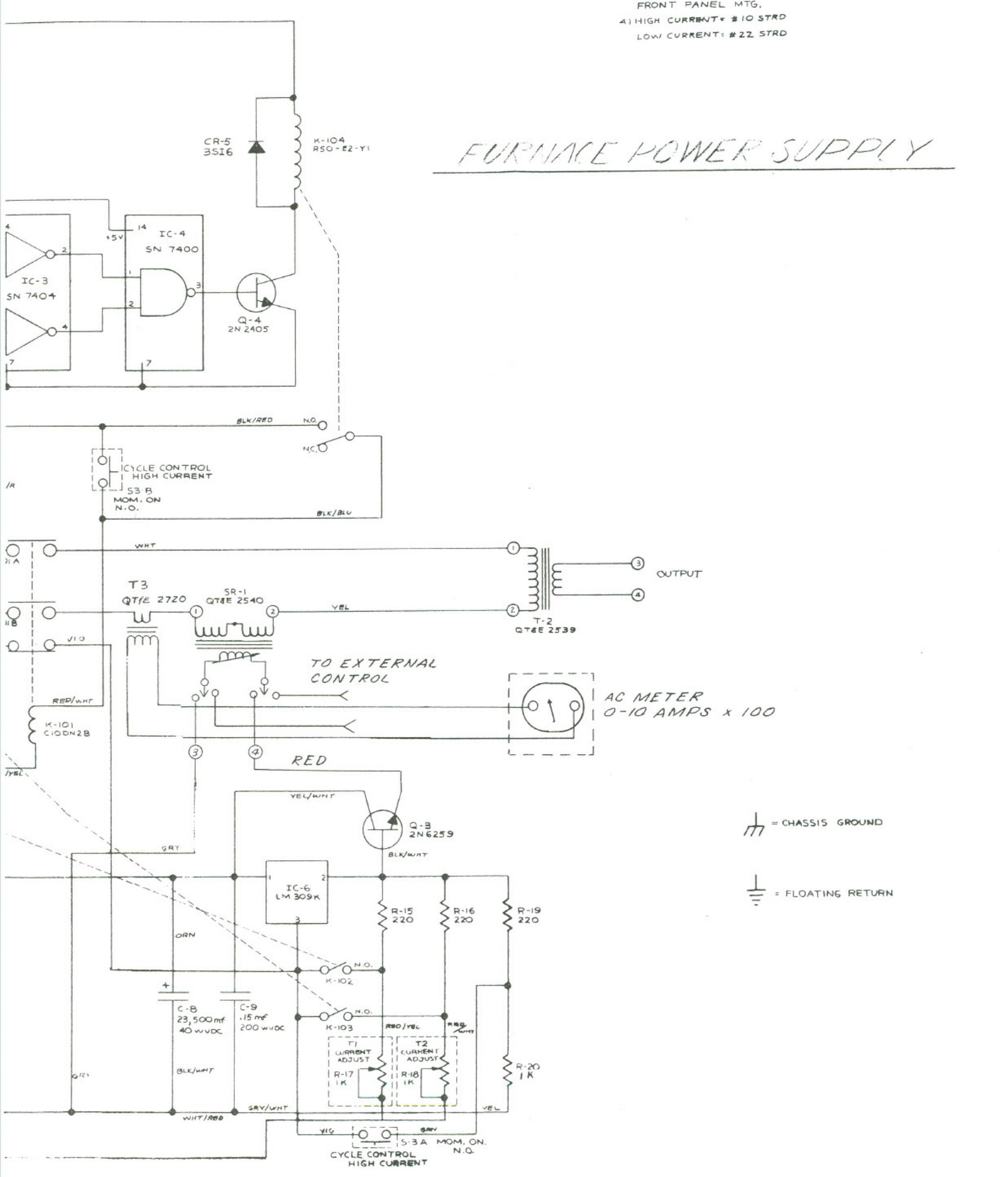




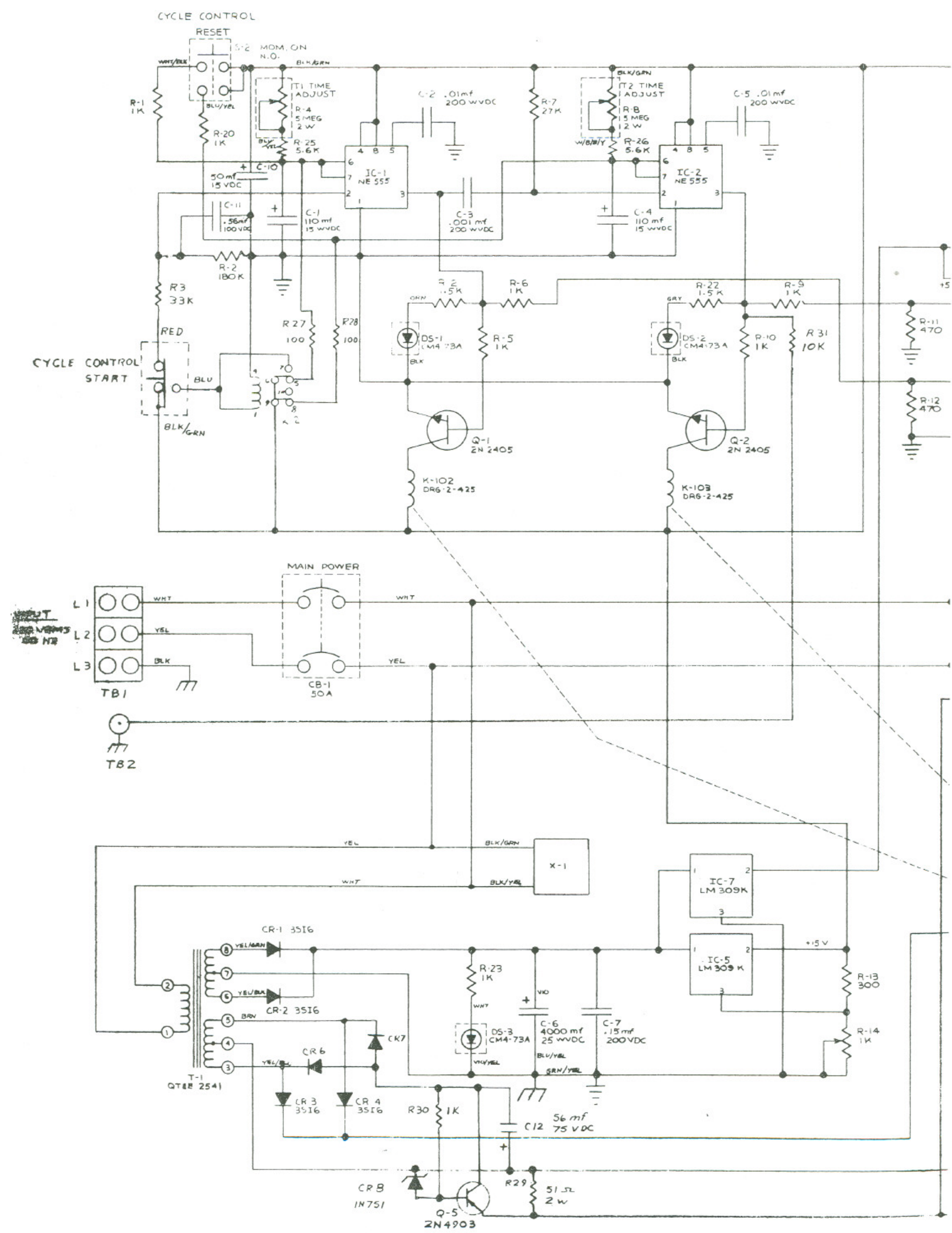


CONTROL LOGIC

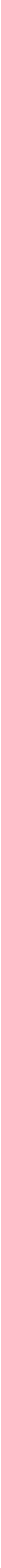


FURNACE

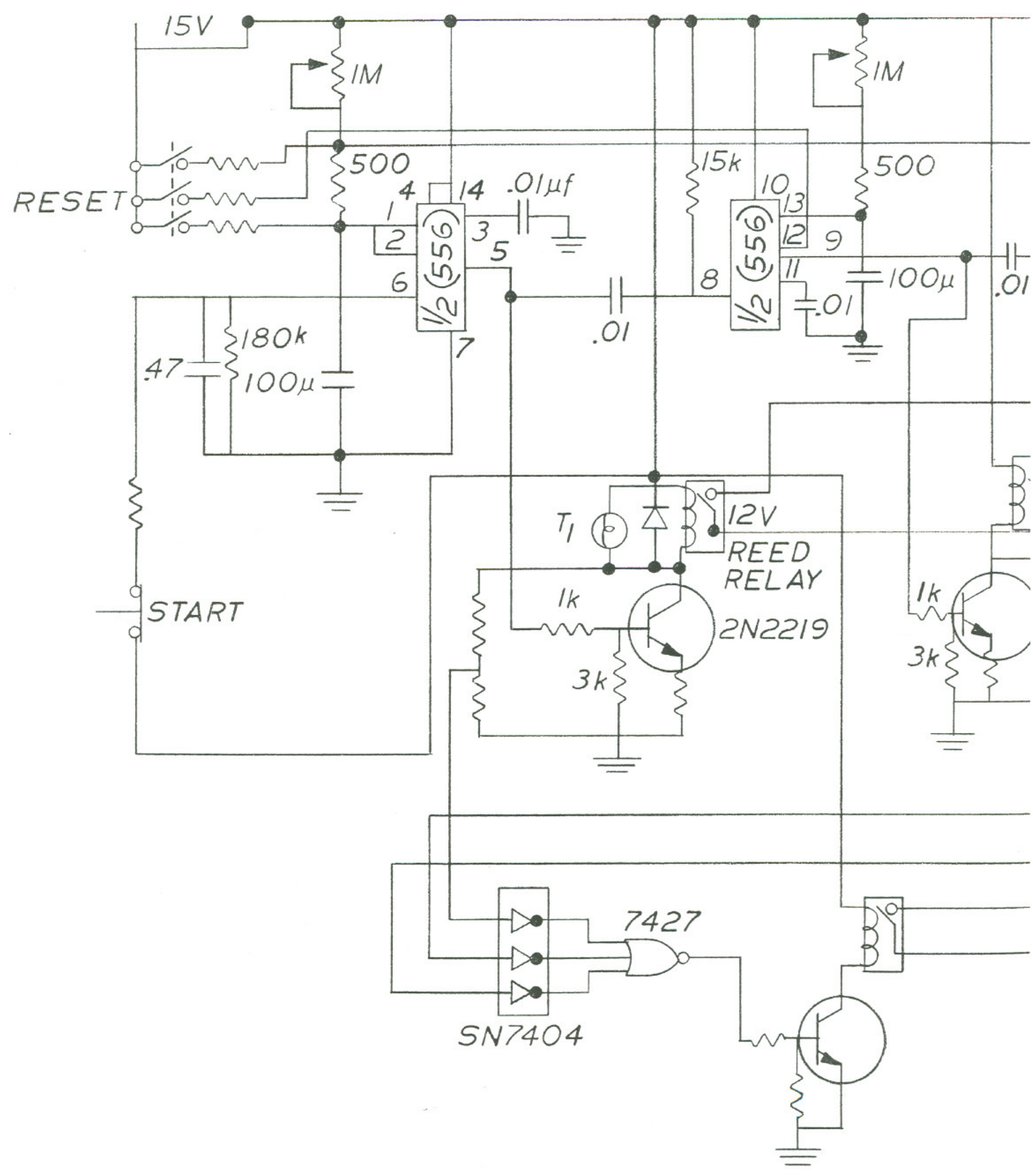


FURNACE CONT

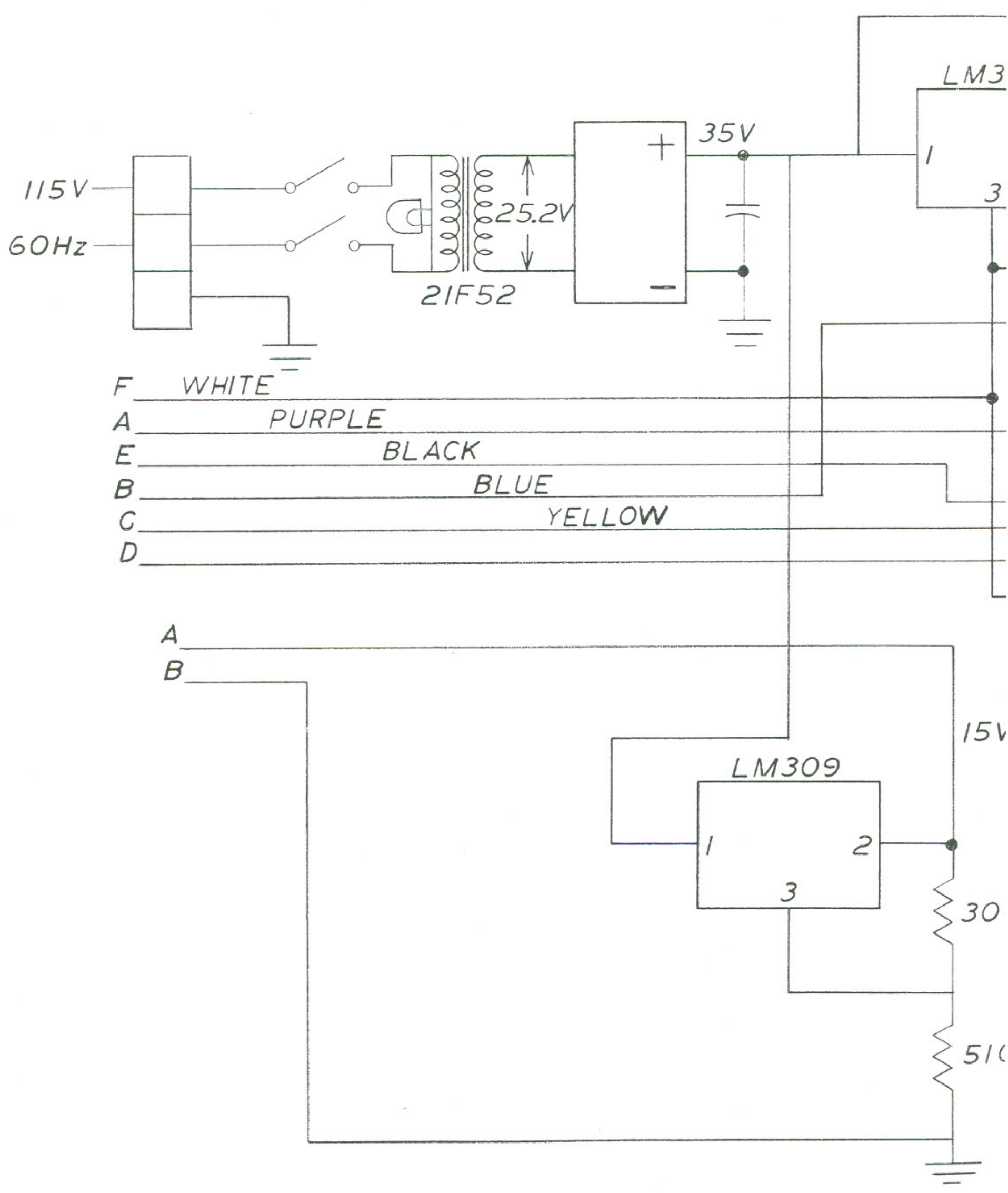




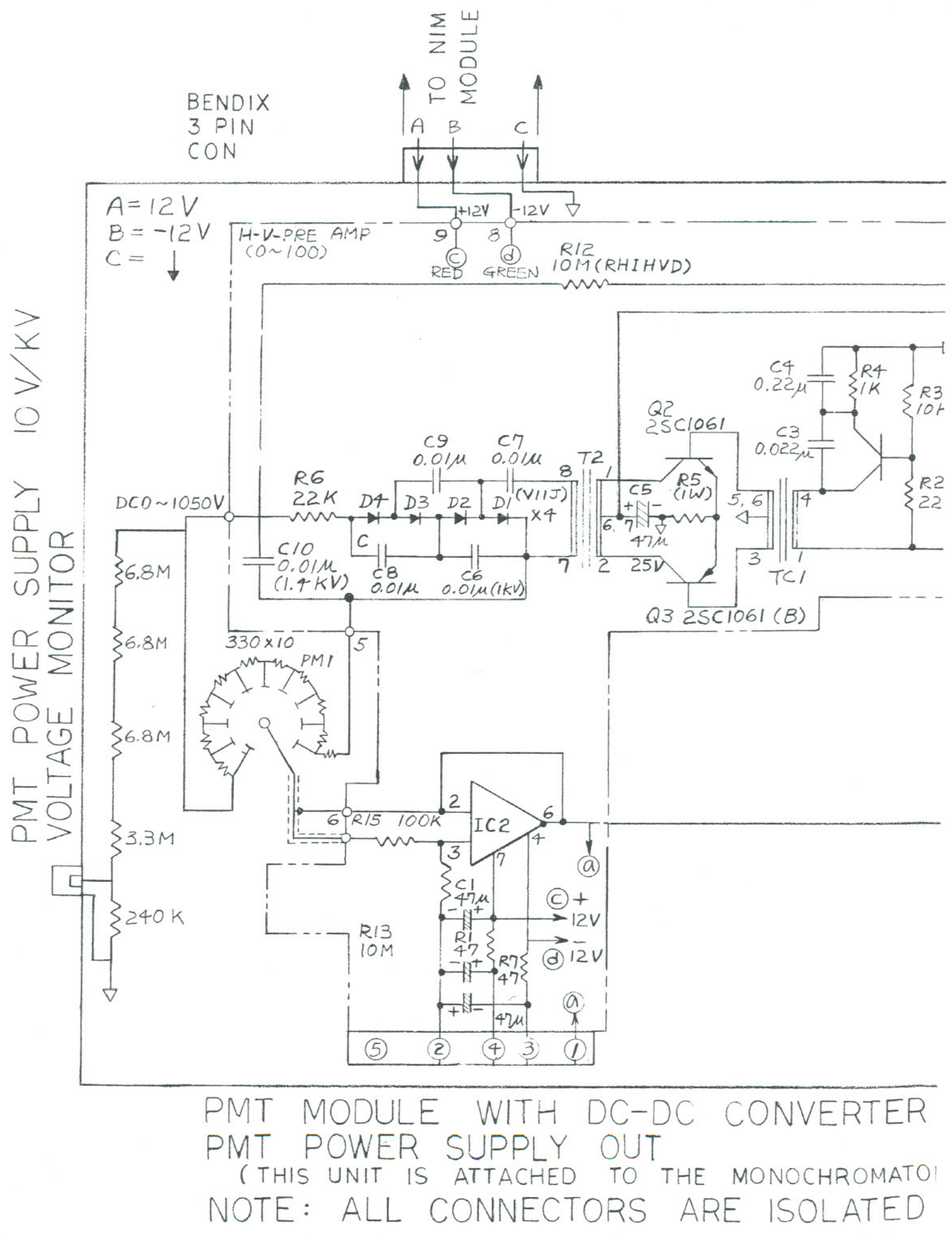




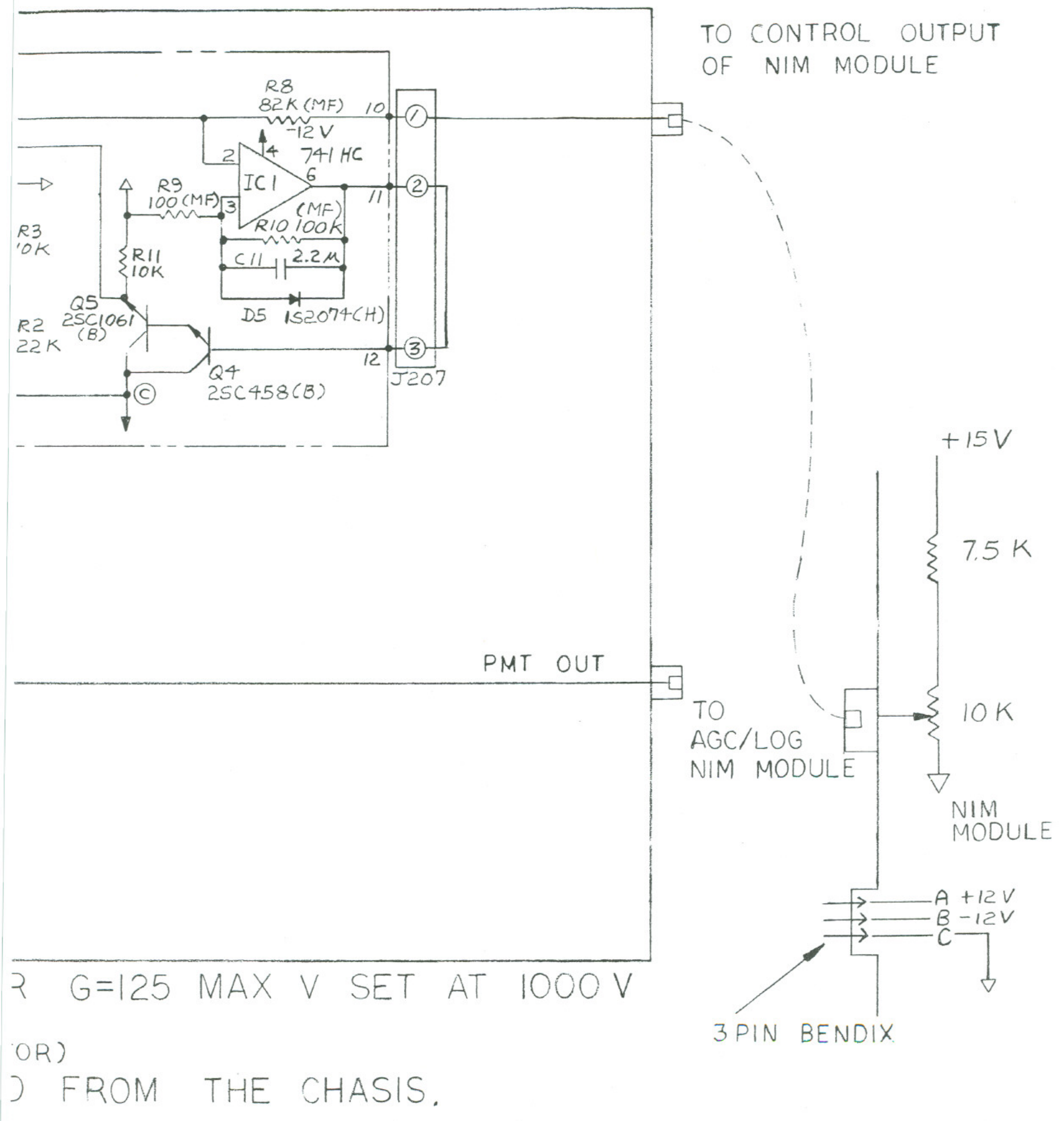




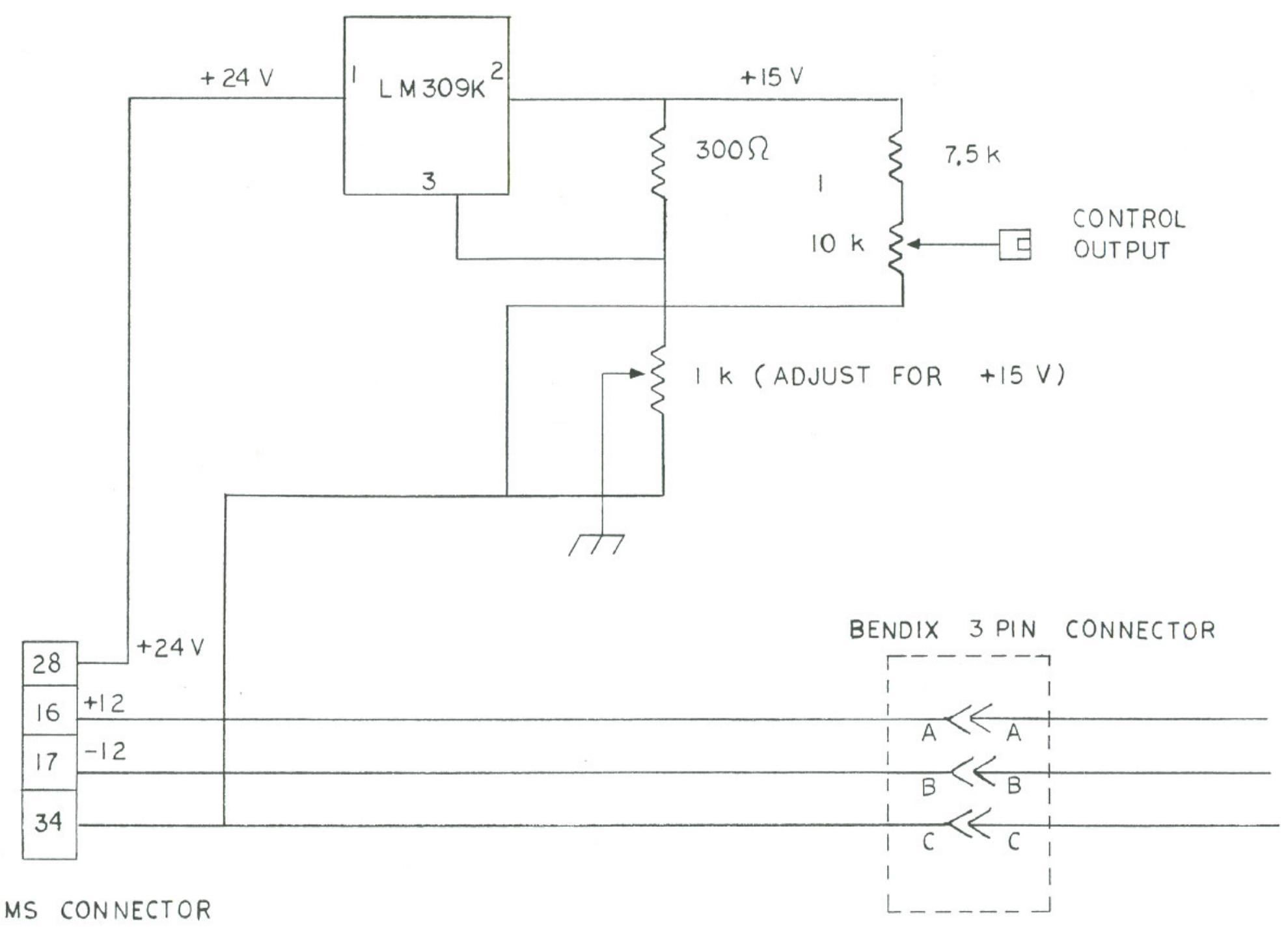




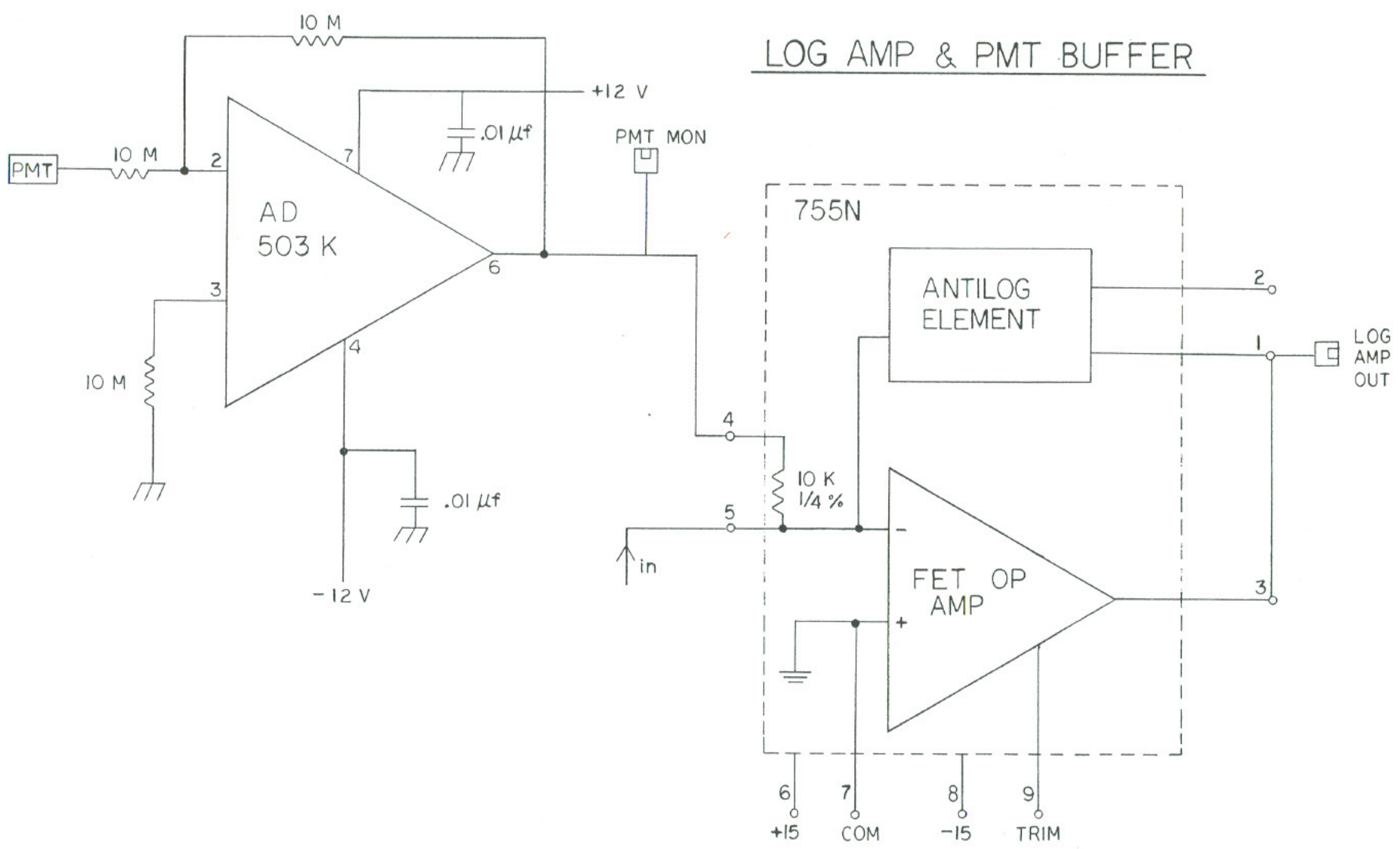



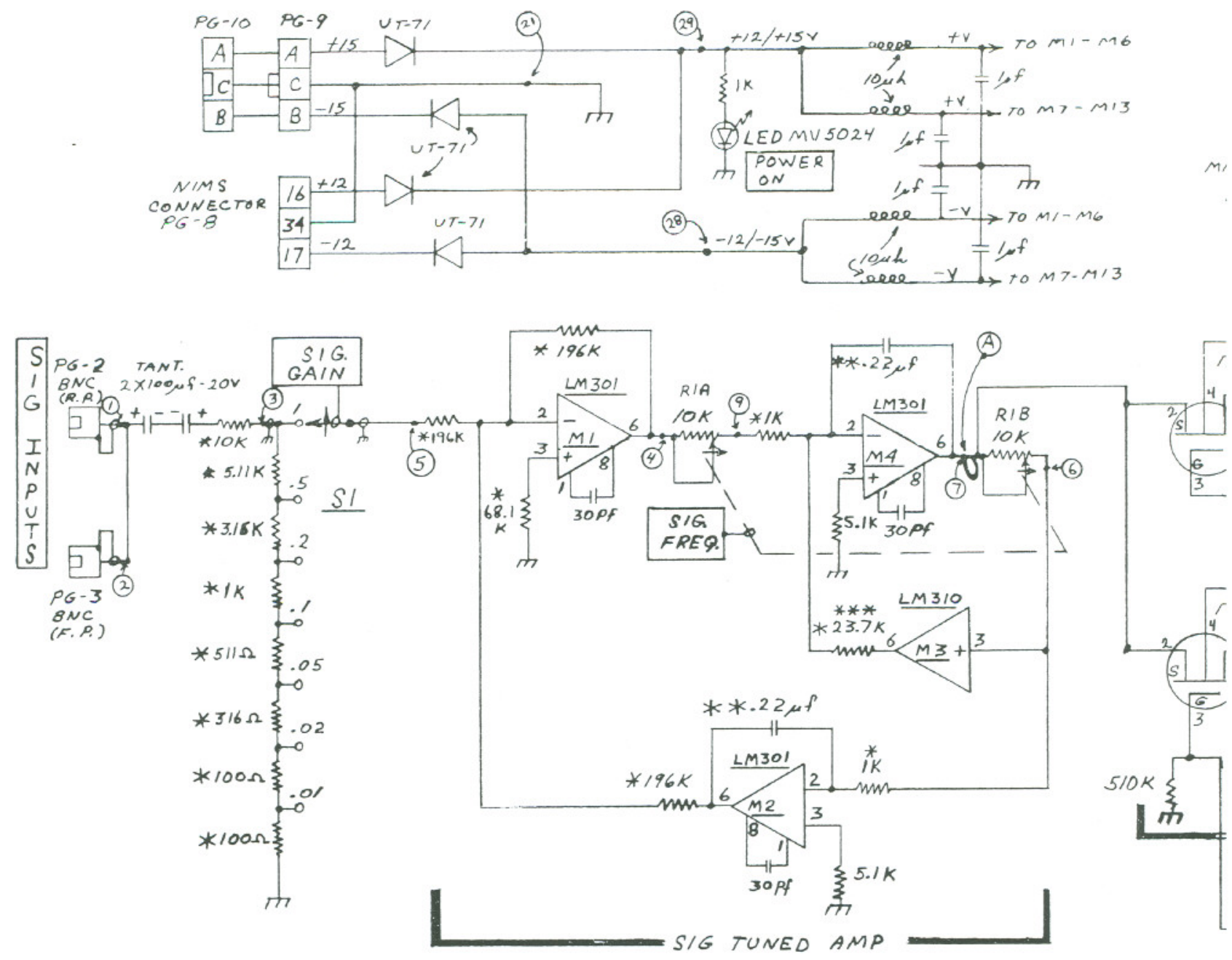

$\Gamma$ PHASE SHIFTER

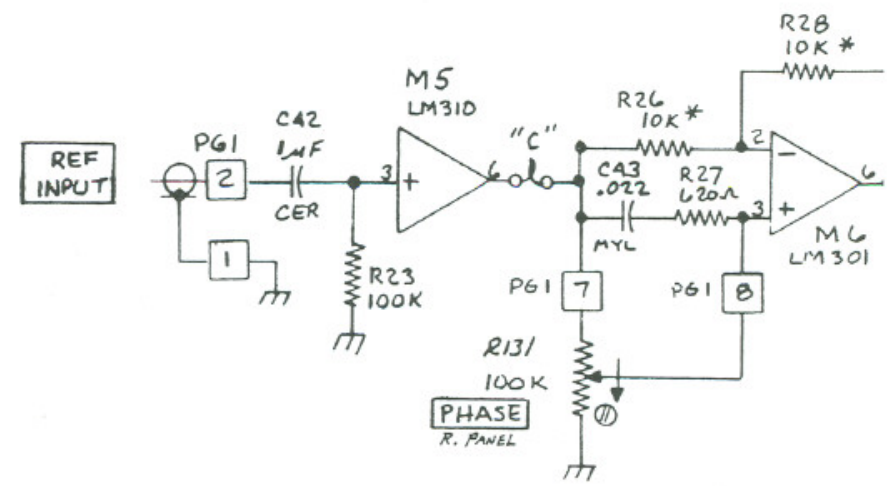




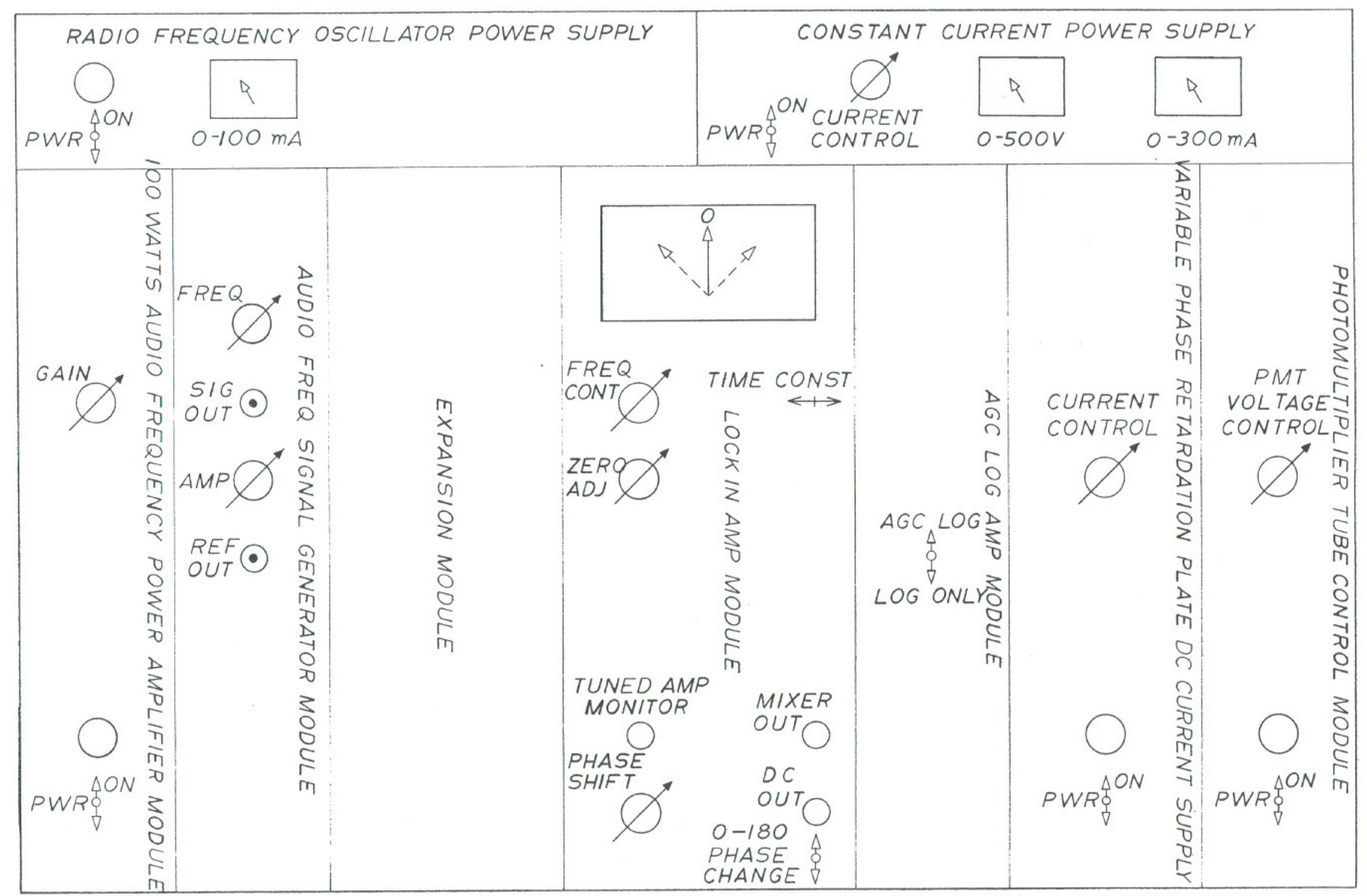

ELECTRONIC MODULE BIN - FRONT VIEW 


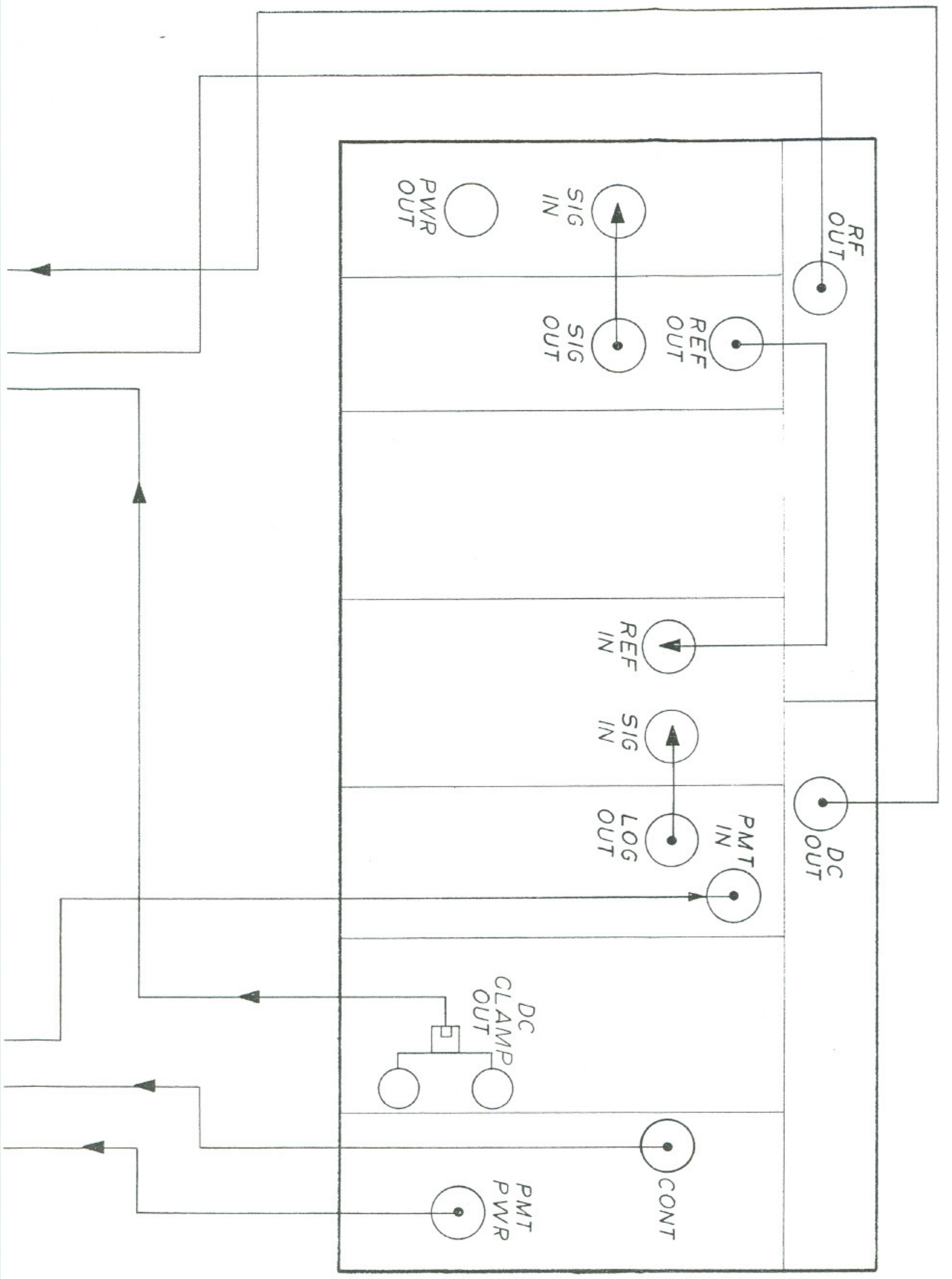

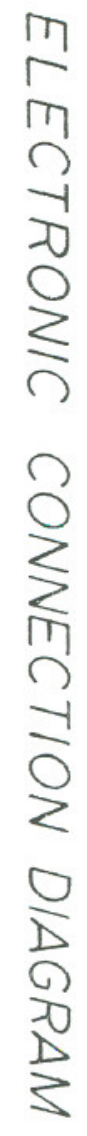




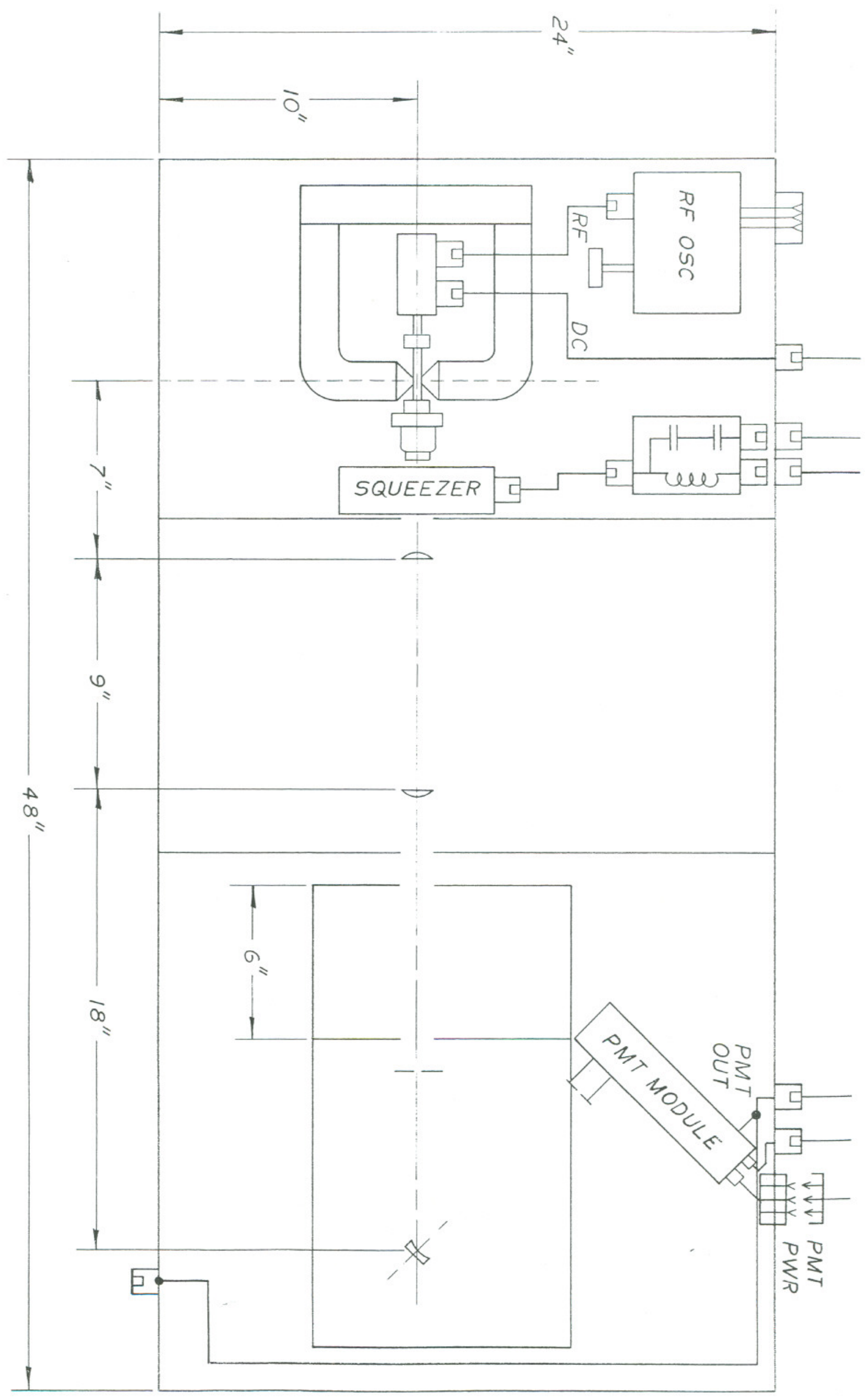




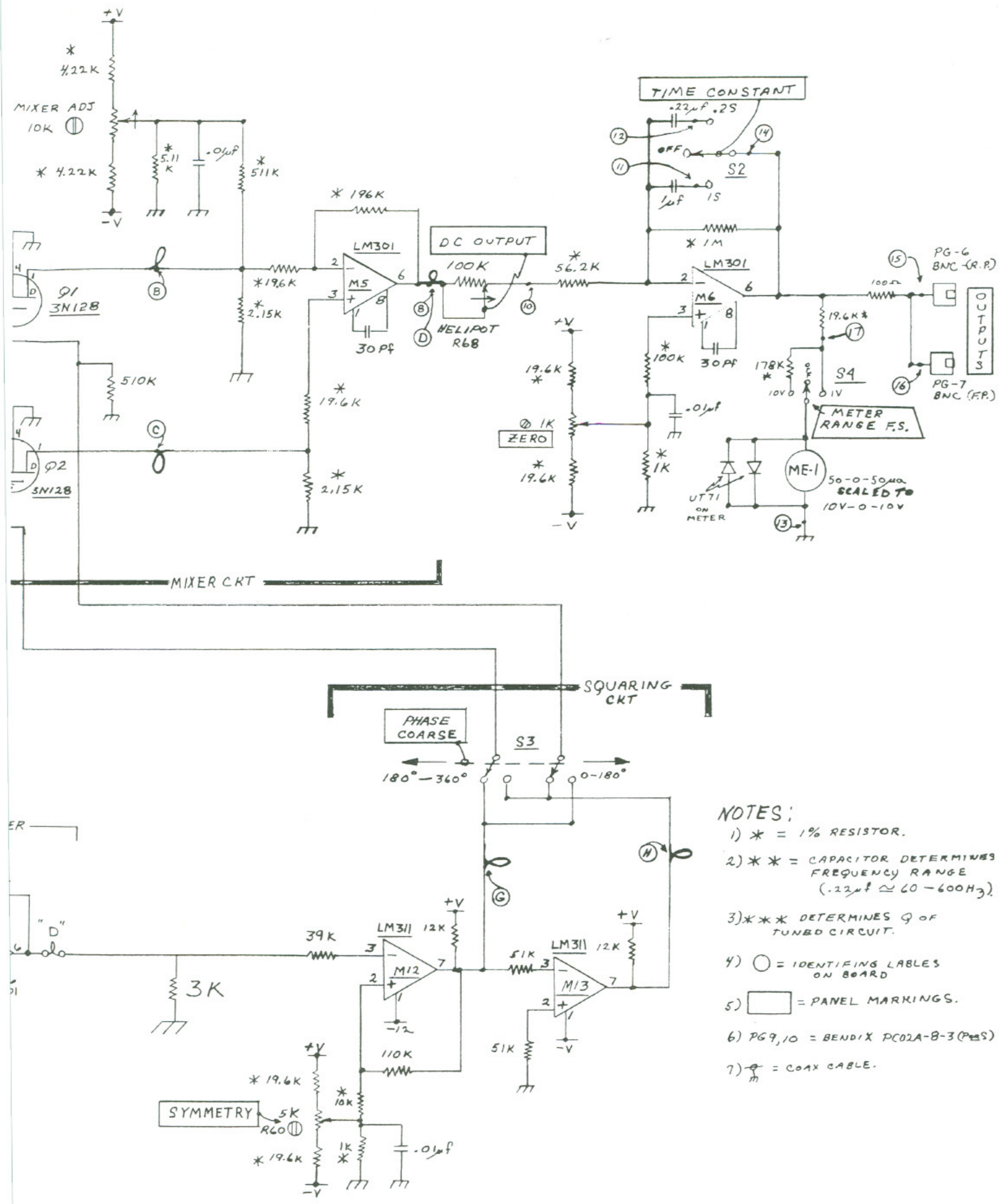

